\title{
Engineering for the ATLAS SemiConductor Tracker (SCT) End-cap
}

\section{A. Abdesselam, ${ }^{y}$ P.P. Allport, ${ }^{o}$ B. Anderson, ${ }^{r}$ L. Andricek, ${ }^{u}$ F. Anghinolfi, ${ }^{e}$} R.J. Apsimon, ${ }^{a c}$ T. Atkinson, ${ }^{t}$ A. Austin, ${ }^{a c}$ H. Band, ${ }^{,}$P. Barclay, ${ }^{a c}$ A. Barr, ${ }^{y}$ L.E. Batchelor, ${ }^{a c}$ R.L. Bates, ${ }^{j}$ J.R. Batley, ${ }^{d}$ G. Beck, ${ }^{q}$ H. Becker, ${ }^{u}$ P. Bell, ${ }^{s}$ W.H. Bell, ${ }^{j}$ A. Belymam, ${ }^{q}$ J. Beneš, ${ }^{a}{ }^{a}$ P. Beneš, ${ }^{a b}$ E. Berbee, ${ }^{v}$ J. Bernabeu, ${ }^{a k}$ S. Bethke, ${ }^{u}$ N. Bingefors, ${ }^{a j}$ J.P. Bizzell, ${ }^{a c}$ Z.J. Blaszczak, ${ }^{g}$ J. Blocki, ${ }^{g}$ J. Brož, ${ }^{z}$ J. Bohm, ${ }^{a b}$ R. Brenner, ${ }^{a j}$ T.J. Brodbeck, ${ }^{m}$ P. Bruckman de Renstrom, ${ }^{g, y}$ R. Buis, ${ }^{v}$ G. Burton, ${ }^{a c}$ J. Buskop, ${ }^{\nu}$ C.M. Buttar, ${ }^{j}$ J.M. Butterworth, ${ }^{r}$ S. Butterworth, ${ }^{a c}$ E. Capocci, ${ }^{a c}$ C. Carpentieri, ${ }^{h}$ A.A. Carter, ${ }^{q}$ J.R. Carter, ${ }^{d}$ M. Chamizo, ${ }^{i}$ D.G. Charlton, ${ }^{c}$

A. Cheplakov, ${ }^{j}$ A. Chilingarov, ${ }^{m}$ S. Chouridou, ${ }^{a d}$ D. Chren, ${ }^{a a}$ M.L. Chu, ${ }^{a h}$ V. Cindro, ${ }^{p}$ A. Ciocio, ${ }^{n}$ J.V. Civera, ${ }^{a k}$ A. Clark, ${ }^{i}$ P. Coe,${ }^{y}$ A.P. Colijn, ${ }^{\nu}$ P.A. Cooke,${ }^{o}$ M.J. Costa, ${ }^{a k}$ D. Costanzo, ${ }^{a e}$ M. Curtis-Rous, ${ }^{a c}$ C. Dabinett, ${ }^{a c}$ W. Dabrowski, ${ }^{f}$ J. Dalmau, ${ }^{q}$ K.M. Danielsen, ${ }^{x}$ S. D'Auria, ${ }^{j}$ I. Dawson, ${ }^{a}$ P. de Jong, ${ }^{v}$ P. Dervan, ${ }^{o}$ E. Dobson, ${ }^{y}$ F. Doherty, ${ }^{j}$ Z. Doležal, ${ }^{z}$ M. Donega, ${ }^{i}$ M. D'Onofrio, ${ }^{i}$ O. Dorholt, ${ }^{x}$ M. Doubrava, ${ }^{a}{ }^{a}$ I.P. Duerdoth, ${ }^{s}$ C. Duisters, ${ }^{v}$ R. Duxfield,${ }^{a e}$ M. Dwuznik, ${ }^{f}$ S. Eckert,${ }^{h}$ L. Eklund,${ }^{a j}$ C. Escobar, ${ }^{a k}$ D.L. Evans, ${ }^{o}$ V. Fadeyev, ${ }^{a g}$ D. Fasching,${ }^{a l}$ L. Feld, ${ }^{h}$ D.P.S. Ferguson, ${ }^{a l}$ P. Ferrari, ${ }^{e}$ D. Ferrere, ${ }^{i}$ J. Fopma, ${ }^{y}$ P. Ford,${ }^{a c}$ R. Fortin, ${ }^{e}$ J.M. Foster,${ }^{s}$ H. Fox, ${ }^{h}$ T.J. Fraser, ${ }^{r}$ J. Freestone, ${ }^{s}$ R.S. French, ${ }^{a}{ }$ J. Fuster, ${ }^{a k}$ B.J. Gallop, ${ }^{c}$ M. Galuska, ${ }^{a}{ }^{a}$ F. Gannaway, ${ }^{q}$ C. García, ${ }^{a k}$ J.E. García-Navarro, ${ }^{a k}$ M. Gibson, ${ }^{a c}$ S. Gibson, ${ }^{y}$ K. Gnanvo, ${ }^{q}$ J. Godlewski, ${ }^{e}$ F. Gonzalez, ${ }^{a k}$ S. Gonzalez-Sevilla, ${ }^{a k}$ M.J. Goodrick, ${ }^{d}$ G. Gorfine, ${ }^{\nu}$ A. Gorisek, ${ }^{p}$ E. Gornicki, ${ }^{g}$ A. Greenall, ${ }^{o}$ D. Greenfield, ${ }^{a c}$ S. Gregory, ${ }^{t}$ A.A. Grillo, ${ }^{a d}$ J. Grosse-Knetter ${ }^{e}{ }^{e}$ C. Gryska, ${ }^{i}$ L. Haddad, ${ }^{j}$ K. Hara, ${ }^{a i}$ M. Harris, ${ }^{a c}$ F.G. Hartjes, ${ }^{v}$ D. Hauff, ${ }^{u}$ B. Hawes, ${ }^{y}$ T. Hayler, ${ }^{a c}$ S.J. Haywood, ${ }^{a c}$ F. Heinemann, ${ }^{y}$ K. Heinzinger, ${ }^{u}$ N.P. Hessey, ${ }^{v}$ C. Heusch, ${ }^{u}$ A. Hicheur ${ }^{a c}$ J.C. Hill, ${ }^{d}$ M. Hodgkinson, ${ }^{a e}$ P. Hodgson, ${ }^{a e}$ T.I. Hollins, ${ }^{c}$ R. Holt, ${ }^{a c}$ J. Homna, ${ }^{v}$ T. Horažiovskýy ${ }^{a}{ }^{a}$ D. Howell, ${ }^{y}$ G. Hughes, ${ }^{m}$ T. Huse, ${ }^{x}$ M. Ibbotson, ${ }^{s}$ Y. Ikegami, ${ }^{k}$ I. Ilyashenko, ${ }^{a f}$ C. Issever,${ }^{y}$ J. Jakúbek, ${ }^{a}$ J.N. Jackson, ${ }^{o}$ K. Jakobs, ${ }^{h}$ R.C. Jared, ${ }^{a l}$ P. Jarron, ${ }^{e}$ P. Johansson, ${ }^{a e}$ D. John, ${ }^{v}$ A. Jones, ${ }^{a c}$ M. Jones, ${ }^{y}$ T.J. Jones, ${ }^{o}$ D. Joos, ${ }^{h}$ J. Joseph, ${ }^{a l}$ P. Jovanovic, ${ }^{c}$ J. Jusko, ${ }^{a}{ }^{a}$ O. Jusko, ${ }^{a a}$ J. Kaplon, ${ }^{e}$ M. Karagoz-Unel, ${ }^{y}$ Ch. Ketterer, ${ }^{h}$ P. Kodyš, ${ }^{z}$ E. Koffeman, ${ }^{v}$ Z. Kohout, ${ }^{a}{ }^{a}$ T. Kohriki, ${ }^{k}$ H. Kok, ${ }^{v}$ T. Kondo, ${ }^{l}$ S. Koperny, ${ }^{f}$ A. Korporaal, ${ }^{v}$ V. Koukol, ${ }^{a}{ }^{a}$ V. Král, ${ }^{a}{ }^{a}$ G. Kramberger, ${ }^{p}$ P. Kubík, ${ }^{z}$ J. Kudlaty, ${ }^{u}$ W. Kuilman, ${ }^{v}$ N. Kundu, ${ }^{y}$ C. Lacasta, ${ }^{a k}$ V. Lacuesta, ${ }^{a k}$ W. Lau, ${ }^{y}$ S.C. Lee, ${ }^{a h}$ 
R. Leguyt, ${ }^{v}$ K. Leney, ${ }^{o}$ S. Lenz, ${ }^{u}$ C.G. Lester, ${ }^{d}$ Z. Liang, ${ }^{a h}$ K. Liebicher, ${ }^{u}$ M. Limper, ${ }^{,}$ L.E. Lindquist, ${ }^{a j}$ S. Lindsay, ${ }^{o}$ V. Linhart, ${ }^{a}$ A. Lintern, ${ }^{a c}$ C. Locket,${ }^{a c}$ M. Lockwood ${ }^{o}$ F.K. Loebinger, ${ }^{s}$ M. Lozano, ${ }^{a}$ I. Ludwig, ${ }^{h}$ J. Ludwig, ${ }^{h}$ G. Lutz, ${ }^{,}$M. Maassen, ${ }^{h}$ D. Macina, ${ }^{i}$ A. Macpherson, ${ }^{e}$ C. MacWaters ${ }^{a c}$ C.A. Magrath, ${ }^{v}$ P. Malecki, ${ }^{g}$ I. Mandić, ${ }^{p}$

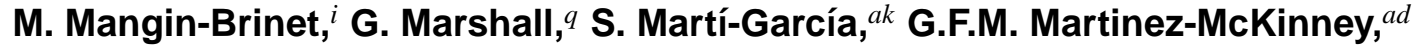
J.P. Matheson, ${ }^{a c}$ F. McEwan, ${ }^{j}$ S.J. McMahon, ${ }^{a c}$ D. McPhail, ${ }^{a c}$ J. Meinhardt, ${ }^{h}$ B. Mellado, ${ }^{a l}$ I.J. Mercer, ${ }^{m}$ I. Messmer, ${ }^{h}$ B. Mikulec, ${ }^{i}$ M. Mikuž, ${ }^{p}$ S. Mima, ${ }^{w}$ K. Mistry, ${ }^{r}$ A. Mitra, ${ }^{a h}$ V.A. Mitsou, ${ }^{a k}$ P. Modesto, ${ }^{a k}$ S. Moed, ${ }^{i}$ B. Mohn, ${ }^{b}$ R. Moles, ${ }^{a k}$ G.F. Moorhead, ${ }^{t}$ B. Moreno, ${ }^{a}{ }^{k}$ J. Morin, ${ }^{q}$ J. Morris, ${ }^{q}$ H.G. Moser, ${ }^{u}$ A. Moszczynski, ${ }^{g}$ A.J.M. Muijs, ${ }^{v}$ B. Munneke, ${ }^{v}$ W.J. Murray, ${ }^{a c}$ D. Muskett, ${ }^{o}$ J. Nacher ${ }^{a k}$ K. Nagai, ${ }^{q}$ D. Naito, ${ }^{w}$ I. Nakano, ${ }^{w}$ C. Nelson, ${ }^{a c}$ A. Nichols, ${ }^{a c}$ R.B. Nickerson,,${ }^{y}$ R. Nisius, ${ }^{u}$ J. Noviss, ${ }^{a c}$ M. Olcese, ${ }^{a m}$ V. O'Shea, ${ }^{j}$ O.K. Oye,${ }^{b}$ S. Paganis, ${ }^{a e}$ M.J. Palmer, ${ }^{d}$ M.A. Parker, ${ }^{d}$ U. Parzefall, ${ }^{h}$ J.R. Pater, ${ }^{s}$ H. Pernegger,${ }^{e}$ E. Perrin, ${ }^{i}$ A. Phillips, ${ }^{d}$ P.W. Phillips, ${ }^{a c}$ J.P. Pieron, ${ }^{g}$ K. Poltorak, ${ }^{f}$ S. Pospíšil, ${ }^{a}{ }^{a}$ M. Postranecky, ${ }^{r}$ T. Pritchard, ${ }^{e}$ K. Prokofiev, ${ }^{a e}$ C. Raine,${ }^{j}$ P.N. Ratoff, ${ }^{m}$ A. Reitmeijer, ${ }^{,}$P. Řezníček, ${ }^{z}$ R.H. Richter, ${ }^{u}$ A. Robichaud-Véronneau, ${ }^{i}$ D. Robinson, ${ }^{d}$ A. Robson, ${ }^{j}$ R. Rodriguez-Oliete, ${ }^{a k}$ S. Roe,${ }^{e}$ G. Rolfe,${ }^{a c}$ J. Rövenkamp, ${ }^{\nu}$ K. Runge, ${ }^{h}$

A. Saavedra, ${ }^{a g}$ H.F.W. Sadrozinski, ${ }^{a d}$ F.J. Sanchez, ${ }^{a k}$ H. Sandaker, ${ }^{x}$ J. Schieck, ${ }^{,}$ H. Schuijlenburg, ${ }^{v}$ J. Siegrist, ${ }^{n}$ A. Seiden, ${ }^{a d}$ A. Sfyrla, ${ }^{i}$ G. Simm, ${ }^{o}$ J. Slatter, ${ }^{m}$ T. Slavíéek, ${ }^{a}$ B. Smith, ${ }^{a c}$ K.M. Smith, ${ }^{j}$ N.A. Smith, ${ }^{o}$ C. Snippe, ${ }^{v}$ S.W. Snow, ${ }^{s}$ M. Solar, ${ }^{a}$ A.O. Solberg, ${ }^{b}$ B. Sopko, ${ }^{a}{ }^{a}$ V. Sopko, ${ }^{a}$ L. Sospedra, ${ }^{a k}$ G.D. Southern, ${ }^{o}$ M. Sowinski, ${ }^{g}$ E. Spencer, ${ }^{a d}$ H. Spieler, ${ }^{n}$ E. Stanecka, ${ }^{g}$ S. Stapnes, ${ }^{x}$ J. Stastny, ${ }^{a b}$ I. Šteckl, ${ }^{a}{ }^{a}$ M. Stodulski, ${ }^{g}$ V. Strachko, ${ }^{a k}$ A. Stradling, ${ }^{a l}$ B. Stugu, ${ }^{b}$ P. Sutcliffe, ${ }^{o}$ R. Szczygiel, ${ }^{f}$ R. Takashima, ${ }^{a l}$ R. Tanaka, ${ }^{w}$ G. Tappern, ${ }^{e}$ J. Tarrant, ${ }^{a c}$ G.N. Taylor,${ }^{t}$ S. Temple, ${ }^{a c}$ P.K. Teng, ${ }^{a h}$ S. Terada, ${ }^{k}$ R.J. Thompson, ${ }^{s}$ N.E. Thresher, ${ }^{o}$ M. Titov, ${ }^{h}$ D.R. Tovey, ${ }^{a c}$ G. Tratzl, ${ }^{u}$ A. Tricoli, ${ }^{a c}$ M. Turala, ${ }^{g}$ P.R. Turner, ${ }^{o}$ M. Tyndel, ${ }^{a c}$ M. Ullán, ${ }^{a}$ Y. Unno, ${ }^{k}$ T. Vickey, ${ }^{a l}$ V. Vacek, ${ }^{a a}$ E. Van der Kraaij, ${ }^{v}$ M. Van Ovenbeek, ${ }^{v}$ G. Viehhauser, ${ }^{y}$ C. Vu, ${ }^{n}$ E.G. Villani, ${ }^{a c}$ T. Vu Anh, ${ }^{i}$ J.H. Vossebeld, ${ }^{o}$ M. Wachler,${ }^{u}$ R. Wallny, ${ }^{e}$ C.P. Ward, ${ }^{d}$ M.R.M. Warren, ${ }^{r}$ R. Wastie, ${ }^{y}$ M. Weber ${ }^{a c}$ A.R. Weidberg, ${ }^{y}$ P. Weilhammer, ${ }^{e}$ P.S. Wells, ${ }^{e}$ P. Werneke, ${ }^{v}$ P. Wetzel, ${ }^{u}$ M.J. White,${ }^{d}$ M. Wiesmann, ${ }^{u}$ I. Wilmut, ${ }^{a c}$ J.A. Wilson, ${ }^{c}$ M. Wolter, ${ }^{g}$ M.P. Wormald, ${ }^{o}$ S.L. Wu, ${ }^{a l}$ X. Wu, ${ }^{i}$ J. Zimmer, ${ }^{u m}$ A. Zsenei ${ }^{i}$ and H. Zhu ${ }^{a e}$

\footnotetext{
${ }^{a}$ Centro Nacional de Microelectrónica de Barcelona, CNM-IMB, CSIC, Barcelona, Spain

${ }^{b}$ Department of Physics and Technology, University of Bergen, N 5007 Bergen, Norway

${ }^{c}$ School of Physics and Astronomy, University of Birmingham, Birmingham, U.K.

${ }^{d}$ Cavendish Laboratory, Cambridge University, Cambridge, U.K.

${ }^{e}$ European Laboratory for Particle Physics (CERN), Geneva, Switzerland

${ }^{f}$ Faculty of Physics and Applied Computer Sciences,

AGH University of Science and Technology, Cracow, Poland

${ }^{g}$ The Henryk Niewodniczanski Institute of Nuclear Physics,

Polish Academy of Sciences, Cracow, Poland

${ }^{h}$ Fakultät für Physik, Albert-Ludwigs-Universität, Freiburg, Germany

${ }^{i}$ Section de Physique, Universite de Geneve, Switzerland
} 
${ }^{j}$ Department of Physics and Astronomy, University of Glasgow, Glasgow, U.K.

${ }^{k}$ KEK, High Energy Accelerator Research Organization,

Oho 1-1, Tsukuba, Ibaraki 305-0801, Japan

${ }^{l}$ Kyoto University of Education, Faculty of Education, 1 Fujinomori-cho, Fukakusa, Fushimi-ku, Kyoto, Japan

${ }^{m}$ Department of Physics and Astronomy, University of Lancaster, Lancaster, U.K.

${ }^{n}$ Lawrence Berkeley National Laboratory, Berkeley, California, U.S.A.

${ }^{o}$ Department of Physics, Oliver Lodge Laboratory, University of Liverpool, Liverpool, U.K.

${ }^{p}$ Jožef Stefan Institute and Department of Physics, University of Ljubljana, Ljubljana, Slovenia

${ }^{q}$ Department of Physics, Queen Mary and Westfield College, University of London, London, U.K.

${ }^{r}$ Department of Physics, University College, University of London, London, U.K.

${ }^{s}$ School of Physics and Astronomy, University of Manchester, Manchester, U.K.

${ }^{t}$ University of Melbourne, Parkville, Victoria 3052, Australia

${ }^{u}$ Max-Planck-Institut für Physik, München, Germany

${ }^{v}$ NIKHEF, Amsterdam, The Netherlands

${ }^{w}$ Okayama University, The Graduate School of Natural Science and Technology,

Tsushima-naka 3-1-1, Okayama 700-8530, Japan

${ }^{x}$ The University of Oslo, Department of Physics, Oslo, Norway

${ }^{y}$ Department of Physics, Oxford University, Oxford, U.K.

${ }^{z}$ Charles University, Faculty of Mathematics and Physics, Prague, The Czech Republic

${ }^{a}$ Czech Technical University, Prague, The Czech Republi

${ }^{a b}$ Institute of Physics of the Academy of Sciences of the Czech Republic,

Prague, The Czech Republic

${ }^{a c}$ Rutherford Appleton Laboratory, Chilton, Didcot, U.K.

${ }^{a d}$ Santa Cruz Institute for Particle Physics, University of California, Santa Cruz, California, U.S.A.

${ }^{a e}$ Department of Physics and Astronomy, University of Sheffield, Sheffield, U.K.

af Ioffe Physico-Technical Institute, St. Petersburg, Russia

${ }^{a g}$ Australian Nuclear Science and Technology Organisation, Sydney, Australia

${ }^{a h}$ Institute of Physics, Academia Sinica, Taipei, Taiwan

${ }^{a i}$ University of Tsukuba, Institute of Pure and Applied Sciences,

1-1-1 Tennodai, Tsukuba, Ibarai 305-8571, Japan

${ }^{a j}$ Uppsala University, Department of Physics and Astronomy, Uppsala, Sweden

${ }^{a k}$ Instituto de Física Corpuscular (IFIC), CSIC-Universidad de Valencia, Valencia, Spain

${ }^{a l}$ Department of Physics, University of Wisconsin, Madison, Wisconsin, U.S.A.

${ }^{a m}$ INFN Genova and Università di Genova, Dipartimento di Fisica,

via Dodecaneso 33, IT-16146 Genova, Italy

E-mail: S.J.Haywooderl.ac.uk 
ABSTRACT: The ATLAS SemiConductor Tracker (SCT) is a silicon-strip tracking detector which forms part of the ATLAS inner detector. The SCT is designed to track charged particles produced in proton-proton collisions at the Large Hadron Collider (LHC) at CERN at an energy of $14 \mathrm{TeV}$. The tracker is made up of a central barrel and two identical end-caps. The barrel contains 2112 silicon modules, while each end-cap contains 988 modules. The overall tracking performance depends not only on the intrinsic measurement precision of the modules but also on the characteristics of the whole assembly, in particular, the stability and the total material budget.

This paper describes the engineering design and construction of the SCT end-caps, which are required to support mechanically the silicon modules, supply services to them and provide a suitable environment within the inner detector. Critical engineering choices are highlighted and innovative solutions are presented - these will be of interest to other builders of large-scale tracking detectors. The SCT end-caps will be fully connected at the start of 2008. Further commissioning will continue, to be ready for proton-proton collision data in 2008 .

KEYWORDS: Particle tracking detectors; Large detector systems for particle and astroparticle physics; Detector design and construction technologies and materials; Overall mechanics design (support structures and materials, vibration analysis etc). 


\section{Contents}

1. Introduction 3

1.1 The ATLAS inner detector

1.2 The SCT end-caps 9

1.3 Requirements 6

1.4 Interfaces 8

1.5 Cooling 9

2. Disks 9

2.1 Bare disks 9

2.1 .1 Design 10

2.1 .2 Choice of Korex $\AA \quad 10$

2.1 .3 Manufacture 11

2.1 .4 Quality control 12

2.1 .5 Properties of the disks 13

2.2 Services 14

2.2.1 Cooling circuits 15

2.2 .2 Other services 20

2.2.3 Services assembly and testing 23

3. Module mounting 26

3.1 Module mounting 26

3.2 Testing 30

4. Support structures 31

4.1 Design 31

4.1.1 Support cylinder 33

4.1 .2 Front and rear supports 34

4.1 .3 Support mechanisms 36

4.1.4 ITE cylinder 36

4.1.5 FEA 37

4.2 Manufacture and testing 38

5. Cylinder services 39

5.1 Evaporative cooling interconnects 40

5.2 Low-mass tapes 41

5.3 Remaining services 42 
6. End-cap assembly 42

6.1 Support of the end-caps

6.2 Disk insertion 43

6.3 Services assembly

6.4 Testing during macro-assembly

7. Transportation of the end-caps 47

7.1 Requirements 47

7.2 Designs of the transportation boxes 48

7.3 Transportation 48

8. Thermal enclosures 49

8.1 Requirements 49

8.2 Design overview 50

8.3 Prototyping 51

8.3 .1 Glue applications 51

8.3.2 Small cylinders 51

8.4 Design and manufacture 52

8.4.1 OTE 52

8.4 .2 Rear thermal pad 53

8.4 .3 ITE 53

8.4 .4 Heater pads

8.4 .5 Membranes 55

8.4.6 FEA studies 56

9. Final assembly and integration with the TRT

9.1 Final assembly of the SCT

9.2 TRT integration 60

9.3 Survey and dry-out 61

10. Installation into ATLAS 62

10.1 Transport and insertion into ATLAS 63

10.2 Installation of services 64

11. Grounding and shielding 68

11.1 Concepts 68

11.2 Measurements 69

12. Status of the end-caps 70

12.1 Status of the hardware 70

12.2 Mass estimates 71

12.3 Expected tracking performance

13. Conclusions 73 
B. Lessons learnt 75

B.1 Layout 75

B.2 Disk services

B.3 Assembly 77

\section{Introduction}

\subsection{The ATLAS inner detector}

The ATLAS inner detector [1, 2] is designed to track charged particles produced in proton-proton collisions at the LHC at an energy of $14 \mathrm{TeV}$. In doing so, it measures their momentum, direction and impact parameters as well as providing some particle identification using transition radiation. The detector is also needed to resolve multiple vertices from the overlap of 23 collisions per bunch crossing expected at design luminosity and the measurement of secondary vertices.

The inner detector consists of the pixel detector with 80 million pixels at the innermost radii, a silicon strip detector (SemiConductor Tracker, SCT) with 6 million strips and outside these a transition radiation tracker (TRT) which contains 400000 straws for charged-particle tracking and transition radiation detection to distinguish between electrons and hadrons. The detector sits in a solenoid magnet [3], which provides at its centre a $2 \mathrm{~T}$ field. The solenoid is contained in a cryostat which also holds the barrel electromagnetic calorimeter. The inner detector is designed to provide precise tracking up to $|\eta|=2.5$, where $\eta$ is the pseudorapidity, defined in terms of the polar angle: $\eta=-\ln (\tan (\theta / 2))$. The layout of the inner detector is shown in figure 1 and figure 8 .

The SCT provides precise tracking at a lower cost per unit area than the pixel detector. The SCT is instrumented with 2112 silicon modules in the barrel and 988 modules in each of the two end-caps [4] (see figure 3). Most modules consist of 4 silicon wafers, each of the order of $6 \mathrm{~cm}$ by $6 \mathrm{~cm}$. Two wafers are bonded together to form strips which are effectively $12 \mathrm{~cm}$ long. The innermost modules on the end-cap disks are shorter, being made of just two wafers. The strip pitch is $80 \mu \mathrm{m}$ in the barrel; the end-cap modules have a key-stone (radial geometry), with an average strip pitch around $80 \mu \mathrm{m}$. The front and back pairs of wafers are rotated by $40 \mathrm{mrad}$ with respect to each other to provide a stereo measurement. The effective precision obtained with a module is around $17 \mu \mathrm{m}$ in the transverse directions and $580 \mu \mathrm{m}$ along the strips [5].

The SCT consists of four instrumented cylinders in the central barrel region and a set of nine instrumented disks in each end-cap. Modules are arranged on the cylinders and disks so that a charged particle originating from the beam-spot envelope crosses at least four layers of SCT modules: the pseudorapidity coverage of the disks starts where the coverage of the cylinders finishes. By design, the two end-caps are intended to be identical.

The parts for the SCT end-caps, such as the modules [П], support structures and services were made in the collaborating institutes. One end-cap (EC-C) was then assembled in the UK by a collaboration of the universities of Glasgow, Lancaster, Liverpool, Manchester, Oxford, Sheffield 


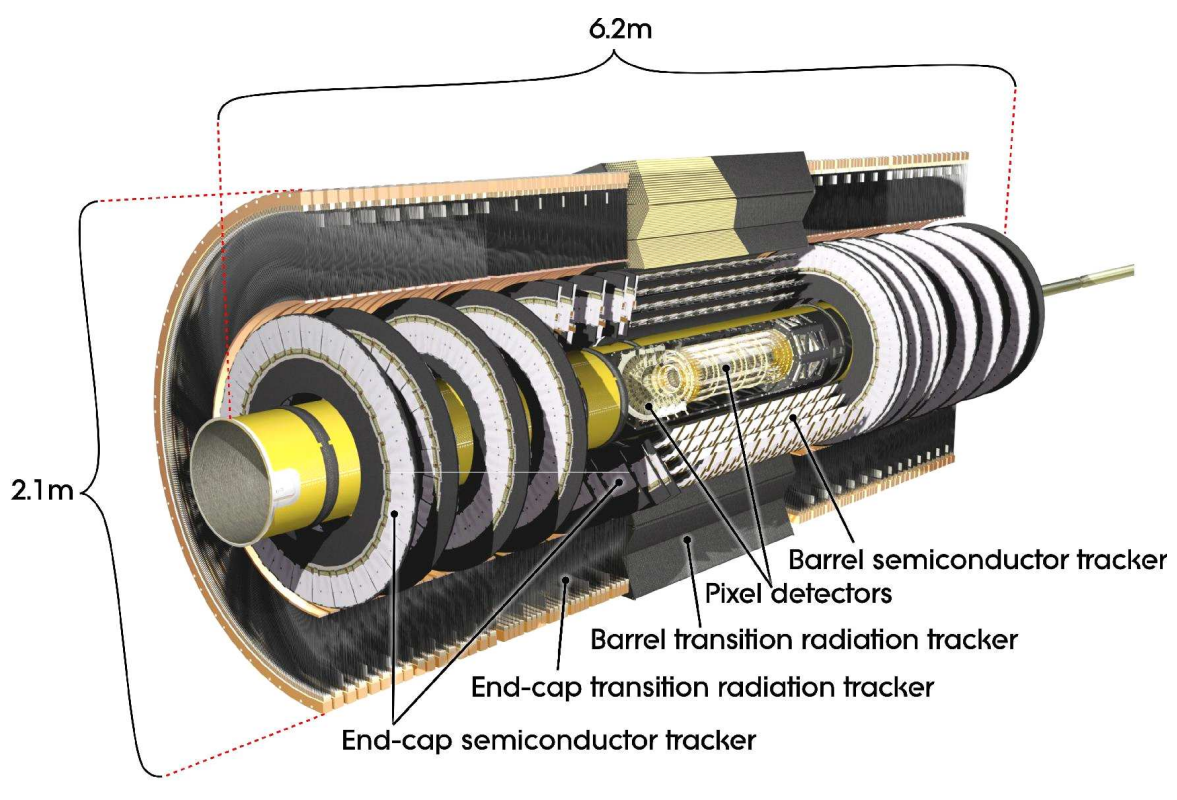

Figure 1. Cutaway view of the ATLAS inner detector.

and the Rutherford Appleton Laboratory (RAL) (part of STFC); the second end-cap (EC-A) was assembled in NIKHEF (Amsterdam, The Netherlands). The end-caps were shipped to CERN where the thermal enclosures and final support mechanisms were added, and the SCT end-caps were integrated with the TRT end-caps before being installed in the ATLAS experiment.

Note on coordinate systems. The z-axis is along the axis of the experiment (in the direction of the colliding beams). In the transverse plane, the $\mathrm{x}$-axis is horizontal and the $\mathrm{y}$-axis is vertical. Cylindrical coordinates $(\mathrm{R}, \phi, \mathrm{z})$ are used and the measurements needed to determine the momentum, denoted by $\mathrm{R} \phi$, are made in the transverse plane, normal to the radial direction.

\subsection{The SCT end-caps}

Each disk has up to three rings of modules: outer, middle and inner. The modules in each ring overlap to avoid gaps in azimuth, and the rings overlap in radius, as seen from the interaction region. The SCT is designed to be hermetic for charged particles of $p_{T}>1 \mathrm{GeV}$. Nevertheless, there are small gaps in the acceptance; in particular, there are only three measurements made in a small transition region between the barrel and each end-cap. The module sizes are designed to optimise the size of silicon detectors cut from circular wafers. This results in 52 modules being required for the outer rings, but only 40 modules for the middle and inner rings.

In each end-cap, the disks are numbered 1 to 9 , starting nearest to the interaction region. It is not necessary for all disks to have outer, middle and inner rings of modules to ensure full coverage. Since a complete middle module is not required on disk 8 to provide coverage up to $|\eta|$ $=2.5$, modules denoted as "short-middles" are used - these only have two silicon wafers. The association of module rings with disks is indicated in table 1, along with the position of the centre of the disk as measured from the nominal beam-spot position [6]. The middle ring is rotated by 

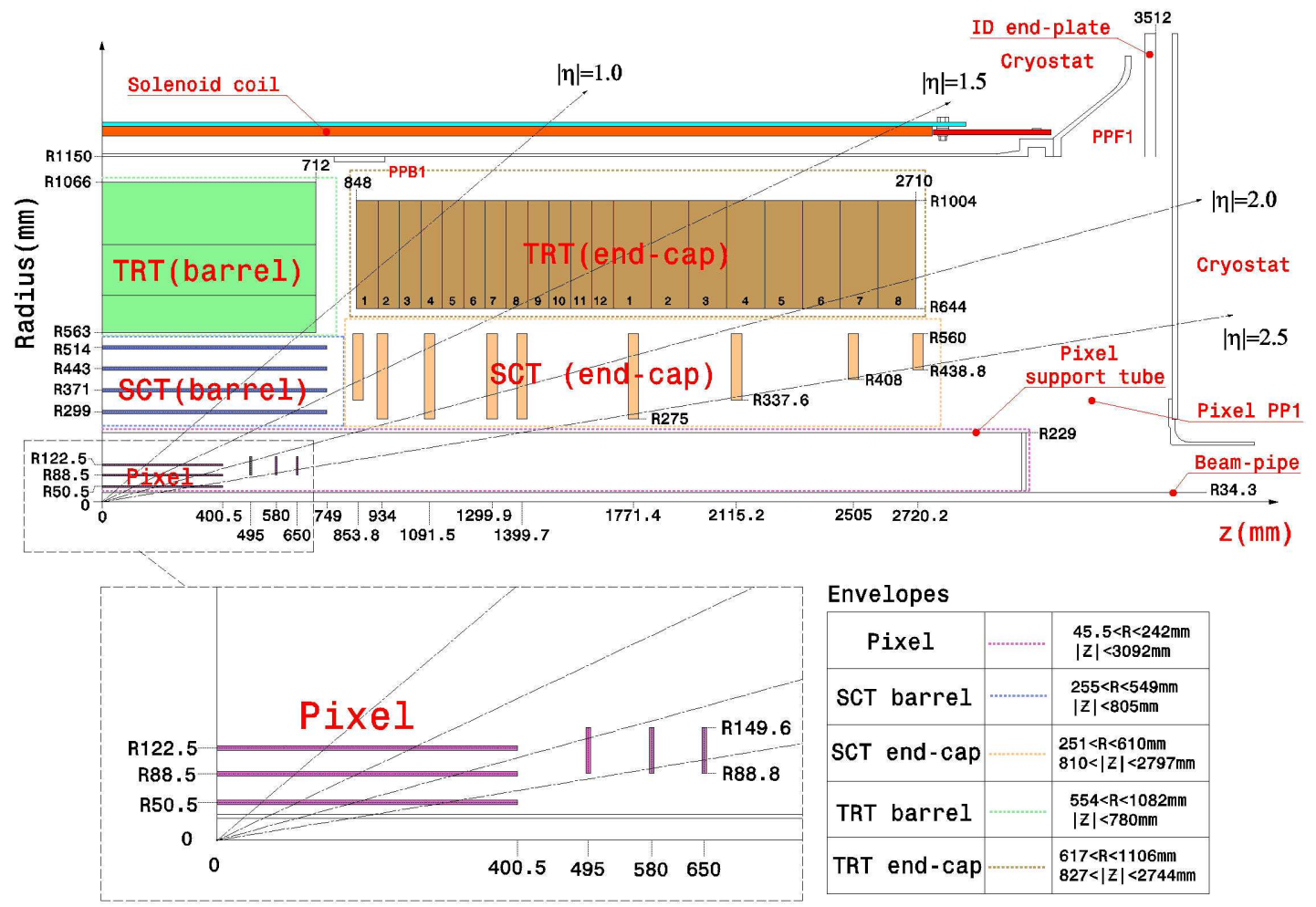

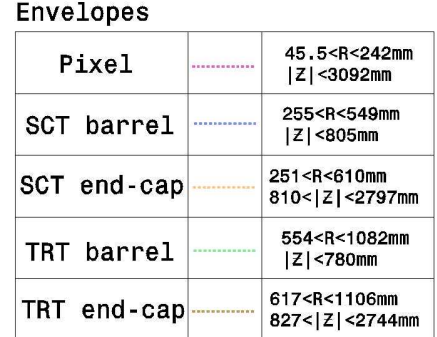

Figure 2. Schematic view of one quarter of the ATLAS inner detector. The SCT disk z-positions are the nominal values for the disk centres; the radii are the radii covered by the silicon modules.

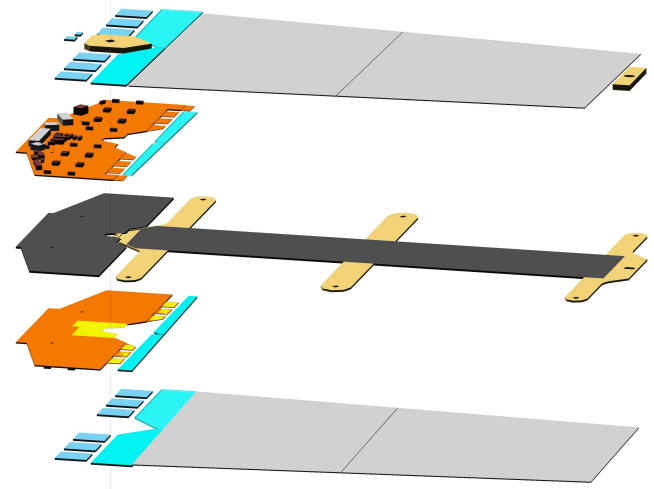

Figure 3. Exploded view of an end-cap outer module.

half a module with respect to the inner ring to avoid the material associated with module cooling blocks lining up in azimuth. 


\begin{tabular}{|l|l|l|l|l|l|l|l|l|l|}
\hline & \multicolumn{6}{|l}{ Disk number } \\
\hline & $\mathbf{1}$ & $\mathbf{2}$ & $\mathbf{3}$ & $\mathbf{4}$ & $\mathbf{5}$ & $\mathbf{6}$ & $\mathbf{7}$ & $\mathbf{8}$ & $\mathbf{9}$ \\
\hline Nominal z position (mm) & 853.8 & 934 & 1091.5 & 1299.9 & 1399.7 & 1771.4 & 2115.2 & 2505 & 2720.2 \\
\hline Stereo angle (mrad) & +40 & -40 & +40 & -40 & +40 & -40 & +40 & -40 & +40 \\
\hline Outer & 52 & 52 & 52 & 52 & 52 & 52 & 52 & 52 & 52 \\
\hline Middle & 40 & 40 & 40 & 40 & 40 & 40 & 40 & & \\
\hline Short-Middle & & & & & & & & 40 & \\
\hline Inner & & 40 & 40 & 40 & 40 & 40 & & & \\
\hline
\end{tabular}

Table 1. Nominal disk positions, stereo angles and the number of modules in each ring.

All the modules in a given ring are exactly the same. To reduce correlations in the measured track parameters and minimise asymmetries, the orientation of the modules alternates. On disk 1, all the modules are rotated by $+20 \mathrm{mrad}$ (clockwise, as viewed from the interaction point), so that the strips of the front layer of silicon are rotated by $+40 \mathrm{mrad}$, while those of the second layer are radial [7]. On disk 2, the rotations are reversed, so that the strips of the front layer of silicon are radial, while those of the second layer are rotated by -40 mrad. For disk 9 , which has modules only on one side, the disk is inserted back-to-front to maximise the rapidity coverage by placing the modules further from the interaction point. This requires that it is built with the same stereo rotations as the even-numbered disks, while the orientation encountered by charged tracks is as if it were an oddnumbered disk. The alternating stereo configurations is realised by rotating the blocks on which the modules are mounted by $\pm 20 \mathrm{mrad}$ from the radial direction for alternately numbered disks.

The disks are held in a large support cylinder which in turn is held by panels at both the front (end nearest to the interaction point) and the rear; these panels attach to the cryostat rails. The end-cap is surrounded by a light-weight thermal enclosure which comprises an outer (OTE) and an inner (ITE) cylinder along with two end panels. The services supplying the modules run on the surface of the disks and along the support cylinder, exiting at the services thermal feed-through and then running in cable trays until they leave the inner detector volume at the edge of the cryostat which holds the solenoid and the electromagnetic calorimeter.

Connections to services are made at the hybrids which are incorporated into the modules, at the PPF0's (forward patch panels) on the outer radius of the disks and at the PPF1's at the end of the cryostat bore.

\subsection{Requirements}

The SCT is designed to provide four space-point measurements for a particle originating from the beam-spot envelope with $|\eta| \leq 2.5$. This provides stand-alone track reconstruction capability with some redundancy.

Placement Precision. The modules are built with internal precisions $\mathrm{O}(10) \mu \mathrm{m}$, and with a tolerance of $5 \mu \mathrm{m}$ in the most critical dimension in the transverse measurement direction [đ]. They are capable of measuring space points to $17 \mu \mathrm{m}$ and so if the uncertainty in the position of the module is not to degrade the track parameter resolution by more than $20 \%$, the position must be understood to better than $12 \mu \mathrm{m}$ in the transverse direction [8]. For precise electroweak measurements, precisions more like $1 \mu \mathrm{m}$ are desirable. The actual position of the modules can be 


\begin{tabular}{|l|l|l|}
\hline \multicolumn{3}{|c|}{ Placement of modules in $\mathbf{R} \phi$ (see section 2.2.3) } \\
\hline Module type & Module placement $(\mu \mathbf{m})$ & Mounting pin location $(\mu \mathbf{m})$ \\
\hline Inner & 67 & 37 \\
\hline Middle & 90 & 60 \\
\hline Outer & 220 & 190 \\
\hline \multicolumn{3}{|c|}{ Placement of disks } \\
\hline & $\mathbf{x}-\mathbf{y}(\mu \mathbf{m})$ & $\mathbf{z}(\mu \mathbf{m})$ \\
\hline & 100 & 1000 \\
\hline
\end{tabular}

Table 2. Placement tolerances. The difference between the module and mounting pin tolerances arises from the tolerances on the location of the mounting washer on the module.

determined during operation from the off-line alignment using particle tracks with high precision $\mathrm{O}(1) \mu \mathrm{m}$ [河]. This reduces the need for very precise placement of the modules and the support structures. Nevertheless, the better the placement, the more readily the alignment procedures will converge and the easier it will be to understand systematic uncertainties. The main requirement for placement comes from ensuring sufficient overlaps between modules to allow precise module-tomodule alignment and to minimise holes seen by particles coming from the interaction point. This requirement is most stringent in the transverse $\mathrm{R} \phi$ direction. Another constraint arises from the requirement to cope with the nominal beam-spot envelope along the axis. The placement tolerances are summarised in table 目 [10].

Stability. If the module position can be monitored using charged tracks with a frequency of once per day, then $1 \mu \mathrm{m} /$ day stabilities are desirable. This may be difficult to achieve for large structures and so the inherent stability will be complemented by internal length measurements within the SCT, determined by a frequency scanning interferometer (FSI) [11-13. The FSI system will measure lengths to $\mathrm{O}(1) \mu \mathrm{m}$ precision several times per hour.

Thermal management. Each module after irradiation will produce up to $10 \mathrm{~W}$ of power [円], resulting in $10 \mathrm{~kW}$ generated in each end-cap. The modules have to be cooled so that the reverse current in the silicon is reduced to a level at which it does not increase the electronic noise. The other important design issue is to avoid thermal runaway which imposes constraints on the thermal impedance between the silicon and the coolant and on the temperature. The optimum temperature to minimise the effects of reverse annealing after irradiation is $-7^{\circ} \mathrm{C}$ [1]. The evaporative cooling system has been designed to keep the SCT at a constant temperature with minimal temperature gradients along the cooling pipes. To minimise the effects of thermal variations in time or space, materials with low coefficient of thermal expansion (CTE) have been used for the support structures.

Coolant. The coolant should be electrically non-conducting and chemically inert in case there are leaks. The fluid should be non-corrosive, non-toxic and non-flammable and stable to the irradiation experienced by the inner detector.

Moisture management. The inside of the SCT will be flushed with dry nitrogen. Before the first data-taking, the SCT will be flushed for several weeks, which will be sufficient to remove most of 
the stored moisture. To minimise the effects of any out-gassing of water vapour, materials with low coefficient of moisture expansion (CME) have been used for the support structures.

Environmental Gas. The SCT detectors will be surrounded by dry nitrogen. Care will be taken to exclude carbon-dioxide (the environmental gas of the TRT and inner detector), which in the presence of water vapour, could form carbonic acid.

Radiation. The materials used must be able to withstand hadron fluences of $2 \times 10^{14} \mathrm{~cm}^{-2} 1 \mathrm{MeV}$ neutron equivalent and an ionising dose of $10^{5} \mathrm{~Gy}(\mathrm{Si})$, over a period of 10 years. The materials used (or ones like them) have all been qualified for exposure to these levels radiation [14]. To allow safe access to the detector during interventions, nuclear activation must be minimised [15]. Silver is particularly troublesome, so local concentrations should be kept to below $\sim 1 \mathrm{~g}$ in a volume corresponding roughly to one module. ${ }^{1}$

Electrical Shielding. To minimise electrical noise pickup from external sources (other components of the inner detector or the LHC machine) and the emission of electrical noise which might affect other detectors, the SCT needs to be shielded by a conductive layer connected to ground; any apertures in the shield should not be more than a few square centimetres. ${ }^{2}$

Materials. All materials must satisfy CERN fire standards [17]; dispensations are required for materials which do not. Magnetic materials which would distort the measured solenoidal field should be avoided. Most magnetic material will saturate in the $2 \mathrm{~T}$ field with a magnetisation of $\mu_{0} \mathrm{M}_{\text {sat }}$. The requirement is that the sum of $\mu_{0} \mathrm{M}_{\text {sat }} \times \mathrm{Vol}$ for all magnetic materials should not exceed $20 \mathrm{~T} \cdot \mathrm{cm}^{3}[18]$.

Mass. To reduce the multiple scattering of charged particles, nuclear interactions, bremsstrahlung of electrons and photon conversions, material must be reduced and where possible, materials with longer radiation lengths should be used. Reducing the mass of components where possible allows the material in the supports to be reduced.

Solenoid quench. The design must be able to cope with quenches of the ATLAS solenoid which produce a peak rate of change of the magnetic field of $0.1 \mathrm{~T} / \mathrm{s}$. To prevent significant forces in the longitudinal direction due to the interaction of transverse eddy currents with the radial field components, conductive loops perpendicular to the field must be avoided.

\subsection{Interfaces}

The SCT end-cap has a defined geometric envelope, with no direct mechanical, electrical or thermal connections to the SCT barrel, the pixels or the TRT. The SCT end-cap is supported by the same rails which support the TRT end-cap, which in turn are supported by rails fixed to the cryostat. To this extent, each SCT end-cap is integrated into a TRT end-cap to provide an assembly which is then installed in the ATLAS detector (see sections $9 \& 10$ ). The pixel detector and its services are

\footnotetext{
${ }^{1} \mathrm{~A}$ module contains $0.2 \mathrm{~g}$ of silver in the conducting glue [15].

${ }^{2}$ The front-end electronics is most sensitive to pick-up in the range 1-10 MHz. Good practice [16] suggests that apertures should be less than $1 / 20^{\text {th }}$ of the corresponding wavelengths, giving dimensions no greater than $\mathrm{O}(1) \mathrm{m}$; multiple apertures will degrade the performance.
} 
held within a carbon-fibre reinforced plastic (CFRP) cylinder: the pixel support tube (PST). The PST is supported by the SCT barrel in the central region and is connected to the inner detector end-plate at the ends.

The SCT has an internal environment of nitrogen, while the inner detector is surrounded by carbon dioxide. The silicon of the SCT barrel is at a similar low temperature of $-7^{\circ} \mathrm{C}$, while the PST facing the inside of the SCT end-caps will be at around $0^{\circ} \mathrm{C}$ and the TRT will be around $22.5^{\circ} \mathrm{C}$.

Originally, it was expected to have a set of TRT wheels behind the SCT end-caps, however these have been dropped from the design; hence this volume will be partly used by the SCT services.

\subsection{Cooling}

SCT modules (and pixel modules) are cooled by evaporating $\mathrm{C}_{3} \mathrm{~F}_{8}$ liquid in cooling pipes which are in good thermal contact with the modules [19]. Liquid is supplied to the detector at the ambient cavern temperature and 14 bar. Capillaries are used as expansion throttling elements, leading to an evaporation temperature of around $-25^{\circ} \mathrm{C}$ in the cooling pipes. In contrast to a pure liquid cooling system, the temperature along the pipes is approximately isothermal, with a small drop of $\sim 2^{\circ} \mathrm{C}$ associated with a reduction in vapour pressure along the pipe.

To make the system more efficient, heat exchangers outside the SCT bring the warm incoming liquid and the cold outgoing vapour into close contact to pre-cool the liquid before it reaches the detector. The system is passive in so far as there is no regulation of the flow-rate of liquid. To ensure that all modules along the cooling circuit can be cooled, there must be excess liquid coolant left at the cooling exhausts. To avoid needing to insulate the exhaust pipes and prevent frost formation, after they exit the controlled, dry volume of the inner detector, heaters beyond the SCT boil off the remaining liquid and bring the pipes to ambient temperature.

\section{Disks}

\subsection{Bare disks}

The end-cap modules are supported on CFRP composite disks comprising two CFRP facesheets and a honeycomb core. These disks also

- Support the module services — see section 2.2.

- Allow handling of the disk, complete with modules, during assembly and integration into the support cylinder.

- Provide attachments to the support cylinder.

The disks are as thin as possible while retaining adequate stiffness. This minimises the gaps between the rings of modules on alternate sides of the disks as seen by tracks coming from a range of collision points within the beam-spot envelope $\left(\sigma_{z}=5.6 \mathrm{~cm}\right)$.

The dimensional requirements are summarised in section 2.1.1; other requirements include [21]: 


\begin{tabular}{|l|l|l|}
\hline Dimension & Value $(\mathbf{m m})$ & Tolerance $(\mathbf{m m})$ \\
\hline Flatness & 0.5 & \\
\hline Thickness & 8.7 & \pm 0.2 \\
\hline Inner radius & 267 & \pm 0.2 \\
\hline Outer radius & 567 & \pm 0.2 \\
\hline Concentricity $^{6}$ & 0.3 & \\
\hline
\end{tabular}

Table 3. Disk design dimensions.

- Dimensional changes in going from assembly conditions $\left(+20^{\circ} \mathrm{C}\right.$ and $50 \%$ relative humidity (RH)) to running conditions $\left(-15^{\circ} \mathrm{C}\right.$ and $\left.<2 \% \mathrm{RH}\right)$ should be less than $250 \mu \mathrm{m} / \mathrm{m}$.

- Dimensional changes in the plane of the disk induced by $\pm 2^{\circ} \mathrm{C}$ and $\pm 0.5 \% \mathrm{RH}$ changes should be less than $10 \mu \mathrm{m} / \mathrm{m}$ in normal running conditions so as not to degrade the alignment.

- The first natural frequency of the bare disk should be higher than $40 \mathrm{~Hz}$ and greater than $15 \mathrm{~Hz}$ for the fully assembled disk.

- Static stiffness (out of plane) should be at least $30 \mathrm{~N} / \mathrm{mm}$.

- The maximum displacement on the statically loaded disk should be less than $10 \mu \mathrm{m}$ in plane and less than $100 \mu \mathrm{m}$ out of plane so as not to invalidate the disk surveys.

- The electrical resistance across the surface of the disk should be less than $0.5 \Omega$.

\subsubsection{Design}

The bare disks were made of $180 \mu \mathrm{m}$ thick CFRP facesheets and a $8.3 \mathrm{~mm}$ thick honeycomb core. The facesheets comprised three plies of high-modulus graphite fibres and cyanate-ester $\operatorname{resin}^{3}$ set at $\pm 60^{\circ}$ to each other. The honeycomb core used was Korex $\AA$ - a high-performance aramid/phenolic honeycomb core ${ }^{4}$ with a cell size of 5/32 inch. The disks were sealed with annular U-profile rings ${ }^{5}$ at the inner and outer radii. The dimensions are summarised in table 3 . The tolerances were proposed following the manufacture of several prototypes and corresponded to what was considered reasonable, while maintaining the stiffness and without increasing the material. The disk thickness and flatness were constrained by the requirements that the disks should be hermetic, given that the module dimensions were already defined.

\subsubsection{Choice of Korex $\mathbb{R}$}

Extensive studies were undertaken to choose an optimal core for the disks [22]. In particular, the

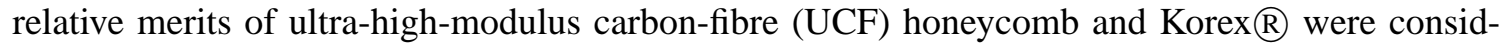

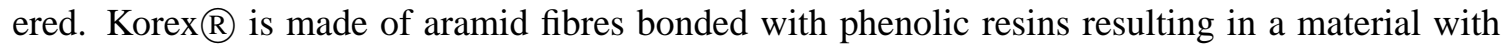

\footnotetext{
${ }^{3}$ YSH-50A graphite fibres in an RS-3 cyanate-ester resin matrix, manufactured by YLA Inc..

${ }^{4}$ Korex-5/32-2.4, manufactured by Hexcel Composite Materials.

${ }^{5}$ T300/RS-3, manufactured by YLA Inc..

${ }^{6}$ See table 5 for definition.
} 


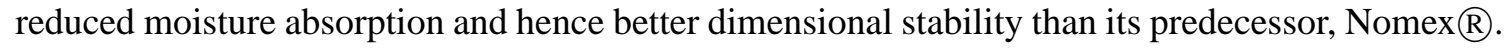
A requirement was a composite capable of withstanding machining forces during manufacture as well as peel, sheer and tensile loadings during use. To compare different composites, the flatwise tensile strength (FWT) was measured. This is a measure of the force required to pull apart a panel laterally: ideally the failure should be in the core, not the glue layers.

The glue used is based on cyanate ester, ${ }^{7}$ since it has low water absorption, typically $0.5 \%$ by mass compared with $6 \%$ for epoxies. As cyanate esters are very sensitive to moisture when curing, and since Korex $\mathbb{R}$ has a high water content on delivery, it was found necessary to dry the core before use.

The FWT's for UCF cores with different glues and different preparations were quite variable: between 0.4 and 1.7 MPa, whereas 2.6 MPa was achieved for Korex $\AA$ with RS4 and 3.6 MPa

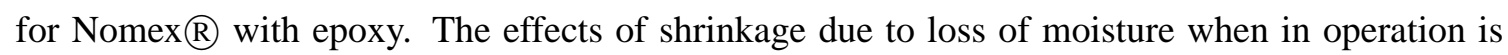

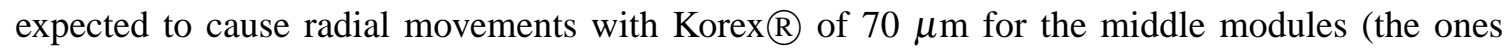
with their mounting points at the highest radii) — this is much less than the tolerance of $200 \mu \mathrm{m}$. Since the stiffness depends on the complete composite, the Korex $\AA$ composites were found to be

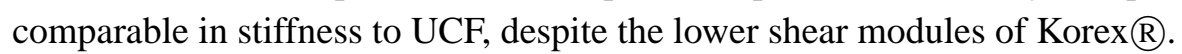

Because of the better bonding achieved with Korex $®$ and the fact that it was $60 \%$ of the cost of a UCF core (a significant fraction of the total cost), Korex $\AA$ was chosen for use in the disks and, to ensure consistent physical properties throughout the end-cap, it was also used for the support structure. Since the Korex $\AA$ came in larger sheets, this resulted in less splicing of sheets to cover the required areas.

\subsubsection{Manufacture}

The disks were manufactured in an autoclave by Programmed Composites Inc. (PCI). The main difficulty was in keeping the flatness tolerance of $0.5 \mathrm{~mm}$. The disks had to be less than $8.7 \mathrm{~mm}$ thick to maintain a radial overlap between middle and outer modules to maintain hermeticity of coverage. The disks have low out-of-plane stiffness, so that small asymmetries in the manufacture (e.g. thermal gradients during curing, or non-flatness of the close-outs) could potentially cause large deformations in disk flatness. The tolerance of $0.5 \mathrm{~mm}$ was necessary to be certain that there would always be adequate clearances between modules and services. PCI achieved a typical flatness over the whole disc of $0.3 \mathrm{~mm}$. To help reference the disks in angle, a yellow aramid fibre was embedded in the surface.

The disks were delivered to NIKHEF where they were made ready for module services [23]. Firstly two reference alignment holes were added at the inner radii. Torlon $\mathbb{R}$ inserts were embedded at 12 points around the outer circumference to allow the disks to be held in the support cylinder - these were tested to $100 \mathrm{~N}$. Next apertures and through holes for inserts were machined using a large reference plate. This plate was used to clamp the disk onto the machine and limit the break-out of the stiff carbon fibres. The apertures, which allow services to cross from one side to the other, were subsequently sealed with moulded CFRP ${ }^{8}$ closeouts.

\footnotetext{
${ }^{7}$ RS4 cyanate-ester adhesive film, manufactured by YLA Inc..

${ }^{8} \mathrm{~T} 300$ graphite fibres in an RS3 cyanate-ester resin matrix, manufactured by YLA Inc..
} 


\begin{tabular}{|l|l|l|}
\hline Dimension & Value & Tolerance \\
\hline Width between insert outer surfaces & $15 \mathrm{~mm}$ & $\pm 0.1 \mathrm{~mm}$ \\
\hline Flatness of insert surfaces & $0 \mu \mathrm{m}$ & $\pm 50 \mu \mathrm{m}$ \\
\hline Slope of insert surfaces relative to disk plane & $0 \mathrm{mrad}$ & $\pm 1 \mathrm{mrad}$ \\
\hline
\end{tabular}

Table 4. Tolerances for the machined disks.

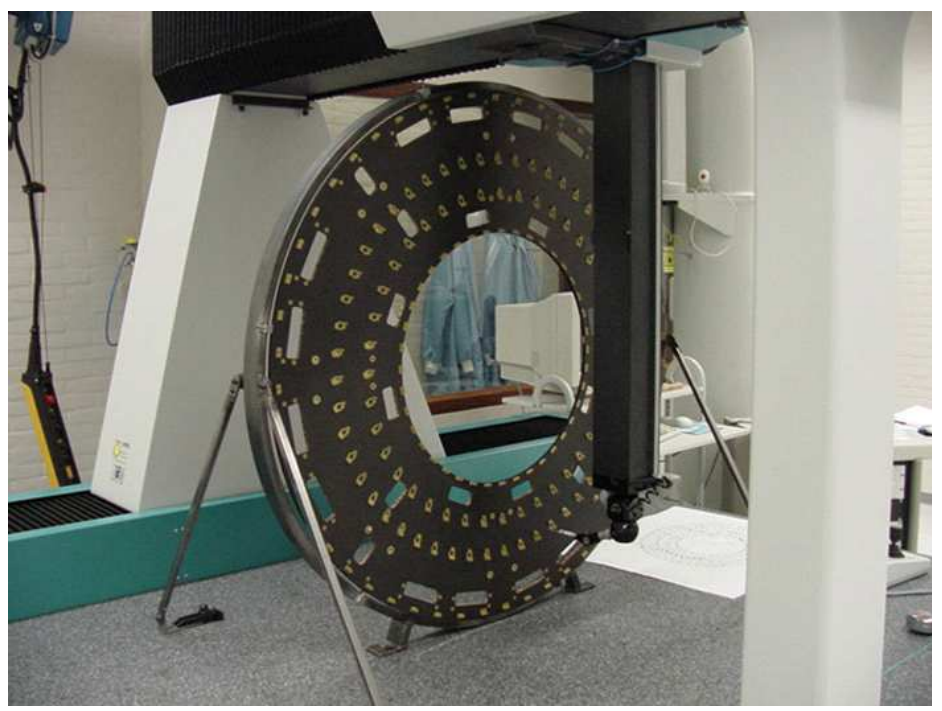

Figure 4. Completed machined disk being measured on a CMM.

Various inserts and pads to provide the attachments for the cooling circuits were added. Jigs were used to place the pads with a tolerance of $300 \mu \mathrm{m}$. The pads were made of Torlon $\AA$ because it is easily machined and very stable against creep. These were glued to the abraded disk surface using epoxy. To ensure the modules are parallel to the disk surface and have sufficient separation between neighbouring modules (the minimum separation between adjacent modules in height is $1 \mathrm{~mm}$ - wirebond to wirebond) and to avoid distorting or stressing the modules, the pad surfaces need to be planar. To achieve the tolerances set out in table 4 , the disk was clamped to a stiff, flat metal plate at $>50$ points across the surface. This was done in the vertical position to avoid bending the disk under its own weight and the temperature of the plate was carefully controlled. The machining was undertaken with the disk horizontal and after each side was machined it was checked on a coordinate measuring machine (CMM) (see figure $\rrbracket$ ), again in a vertical position.

\subsubsection{Quality control}

The bare disks were checked at NIKHEF for stiffness, resonant frequency, weight, and geometrical tolerances. Geometrical tolerances were measured with a 3D CMM. The disks were held vertically during measurement so that they did not deform under their own weight - see figure 9 . They were mounted kinematically by three points and then an additional three support points were added without distorting the disk. Table 5 summarises the measured properties of the disks. Many of the envelope dimensions were just outside the tolerances; however, since the procedure for making the 


\begin{tabular}{|c|c|c|}
\hline Property & Mean & R.m.s. \\
\hline Mass (g) & 1210 & 30 \\
\hline Natural frequency $(\mathrm{Hz})$ & 42 & 2 \\
\hline Static stiffness (N/mm) & 32 & 1 \\
\hline Flatness (front and rear) $(\mu \mathrm{m})$ & 350 & 85 \\
\hline Thickness $(\mathrm{mm})$ & 8.82 & 0.04 \\
\hline Width between insert outer surfaces $(\mathrm{mm})$ & 15.05 & 0.07 \\
\hline Inner radius $(\mathrm{mm})$ & 266.97 & 0.07 \\
\hline Outer radius $(\mathrm{mm})$ & 567.09 & 0.03 \\
\hline Inner circularity ${ }^{9}(\mathrm{~mm})$ & 0.10 & 0.05 \\
\hline Outer circularity $(\mathrm{mm})$ & 0.13 & 0.08 \\
\hline Concentricity ${ }^{10}(\mathrm{~mm})$ & 0.08 & 0.06 \\
\hline Resistance $(\Omega)$ & 0.5 & 0.1 \\
\hline
\end{tabular}

Table 5. Properties of the machined disks.

disks flat was complex and further machining risked damage, the acceptance criteria were relaxed slightly. The insert flatness and slopes were inspected during the measurements and found to be good.

The positions of the mounting pads were measured for all disks. These measurements were repeated at RAL [24] for the first two disks to ensure there had been no deformations during transportation. All the pads measured were well within tolerance $(<300 \mu \mathrm{m})$. An example of the displacements of the pads is shown in figure 5. For the example of disk $9 \mathrm{C},{ }^{11}$ after allowing for global rotation of all the pad positions with respect to the dowel holes, the rotation of the main (secondary) pads had an r.m.s. of 49 (44) $\mu \mathrm{m}$ and maximum values of 100 (150) $\mu \mathrm{m}$.

\subsubsection{Properties of the disks}

A number of prototype disks were made to test the manufacturing techniques and confirm the finite element analysis (FEA) models [25]. Initial calculations of the frequency were found to be $20 \%$ higher than the measured values. Adapting the model then gave a good description of the measured stiffness. The FEA model of the final disk design included the apertures, closeouts, inserts and services and modules. The first natural frequency is a "conical" mode at $22 \mathrm{~Hz}$, shown in figure 6. In-plane displacements are mostly due to the CTE and CME effects, leading to expected movements of $230 \mu \mathrm{m} / \mathrm{m}$. When going from room temperature and a relative humidity of $50 \%$ to the cold and dry environment for operation, out-of-plane distortions not exceeding $40 \mu \mathrm{m}[26]$ are expected to arise from the force of the cooling pipes (see section 2.2.1), since the circuits are assembled at room temperature and yet operate around $-20^{\circ} \mathrm{C}$. Variations in the operating conditions of $2^{\circ} \mathrm{C}$ will result in displacements of only a few microns and the in-plane displacements of the statically loaded disk will be only a couple of microns.

\footnotetext{
${ }^{11}$ The "C" indicates that this disk belongs to EC-C rather than EC-A.
} 


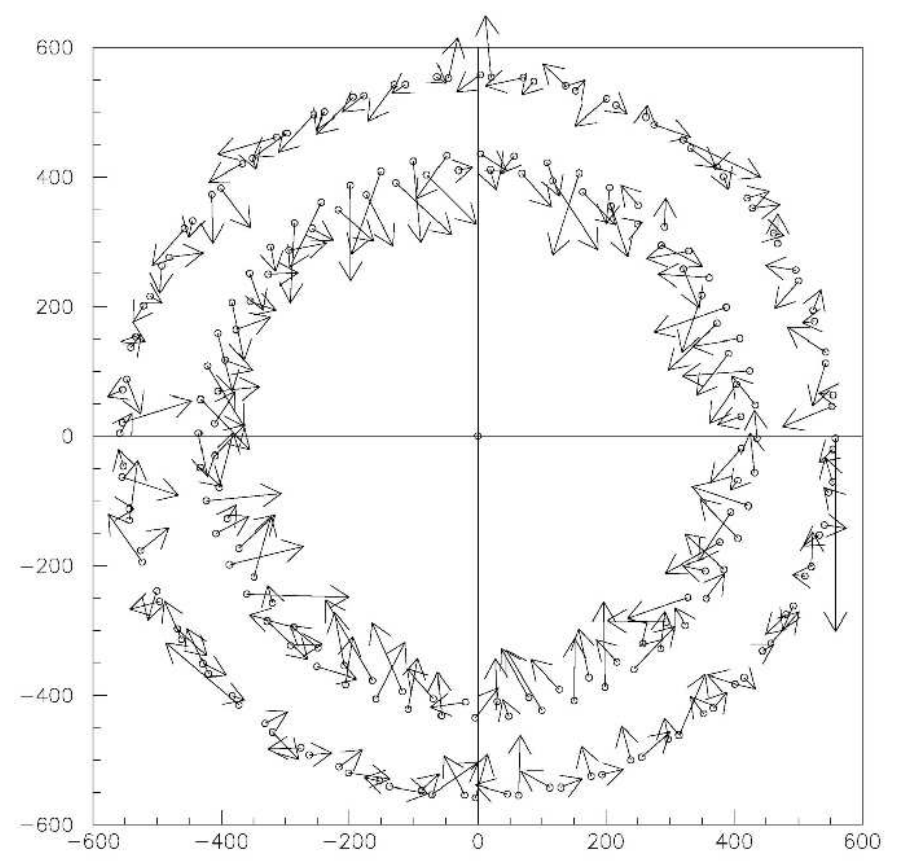

Figure 5. Displacements of pads from their nominal position for main and secondary pads (outer modules) for disk 9C. The positions are in mm, and the displacements are magnified by 1000 .

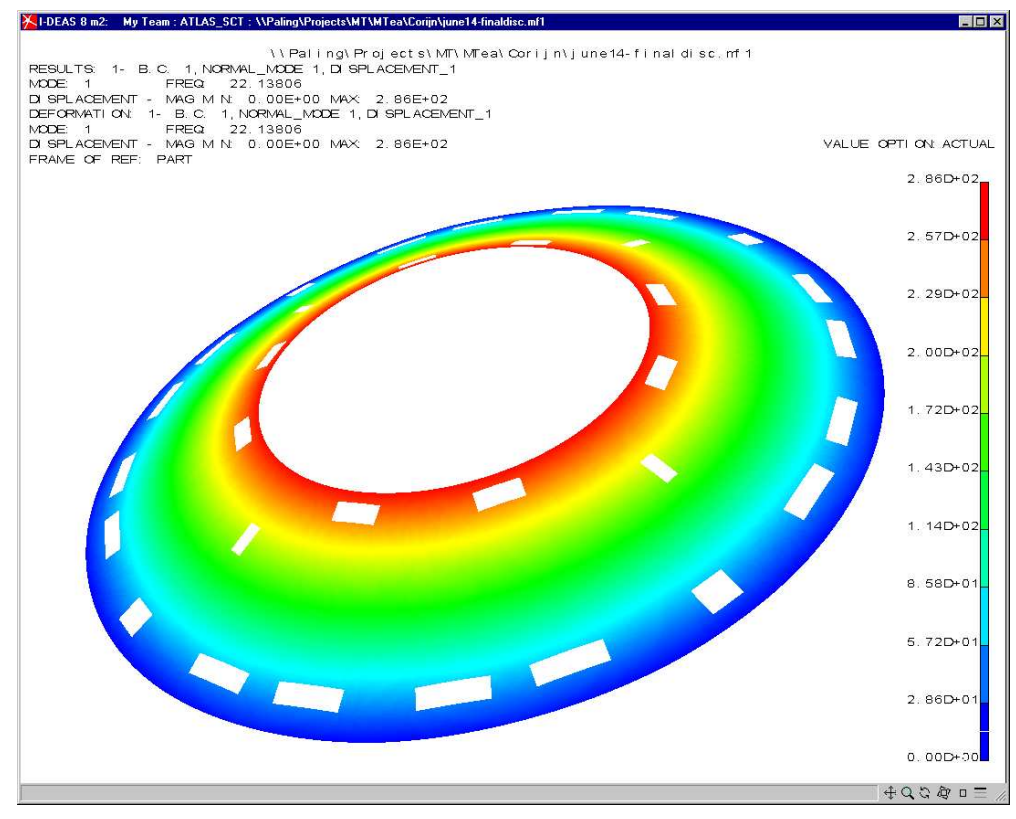

Figure 6. First vibrational mode of a disk.

\subsection{Services}

The modules are held on the disks by means of the blocks which are cooled (see section 2.2.1). Each 
module has electrical power supplied by a "wiggly" power tape (see section 2.2.2.1); it receives timing, trigger and control (TTC) information from one optical fibre (TX) and is read out by 2 optical fibres $(\mathrm{RX})-2$ fibres increase the band-width and provide redundancy.

Each disk is instrumented with its own services [27] which terminate at the outer radius of the disk at a set of patch-panels denoted generically as PPF0. This allows disks to be tested before and after the modules are mounted, since repairs to the services become very difficult after modules are mounted. The services for a disk with all module rings are shown in figure 7. As a general rule for most components (including the CFRP disks), 10-20\% spares were created - this approach worked very well.

There are variations in the services required for each disk depending on which rings of modules it carries. The stereo rotations of the modules (described in section 1.2) also cause differences in the services layout between even-numbered and odd-numbered disks. Fortunately, this only affects the cooling circuits which are rigid; the other services are flexible and can accommodate small movements in the location of the module hybrids $\mathrm{O}(1) \mathrm{mm}$.

\subsubsection{Cooling circuits}

\subsubsection{Design}

The circuits are required to keep the silicon of the SCT modules at $-7^{\circ} \mathrm{C}$. They are manufactured from copper-nickel (Cu-Ni) 70:30 tubing with $70 \mu \mathrm{m}$ wall thickness and $3.74 \mathrm{~mm}$ outer diameter. $\mathrm{Cu}-\mathrm{Ni}$ was chosen since it is fairly resilient to corrosion and easily joined by standard soldering techniques.

There is a separate circuit for each quadrant of each ring, giving either 13 (outer ring) or 10 (middle $\&$ inner rings) modules per circuit. The circuits are manifolded together on the exhaust side at PPF0, resulting in quadrant modularity on each disk. Because cooling circuits are fixed rigidly to the disks, they are stress-relieved by making them "wiggly", as illustrated in figure 8 . Cooling is supplied to the module in the region of the hybrid by a section of the circuit which is approximately radial. In the case of the outer and middle modules, cooling is also supplied at the far end by a circumferential section of the circuit. For the inner modules, no far end cooling is provided due to the modules having approximately half the area of silicon and no mid-point break, hence additional cooling was deemed unnecessary.

Cooling is supplied to the modules via cooling blocks: "main" cooling blocks in the vicinity of the hybrids, and "secondary" cooling blocks at the far end of the modules. These blocks are attached to the cooling circuit by soft solder ${ }^{12}$ and screwed down to the CFRP disk. In turn, the modules are attached firmly to the blocks. The blocks are made from carbon-carbon ${ }^{13}-\mathrm{a}$ form of graphite with high thermal conductivity in one plane (typically $\sim 100 \mathrm{~W} / \mathrm{m} \cdot \mathrm{K}$ ) and poorer conductivity out of the plane $(\sim 50 \mathrm{~W} / \mathrm{m} \cdot \mathrm{K})$. The blocks have an aluminium threaded pin over which the module is placed and secured by a Belleville washer ${ }^{14}$ and small nut. The module hybrids generate more heat than the silicon detectors and the module design has attempted to maximise the thermal isolation of the two sections of the module. This isolation is ensured in the cooling

\footnotetext{
${ }^{12}$ Castolin 197 flux, 70:30 lead-tin.

${ }^{13}$ Manufactured by Dunlop.

${ }^{14}$ Conical washer, capable of providing a small spring force.
} 


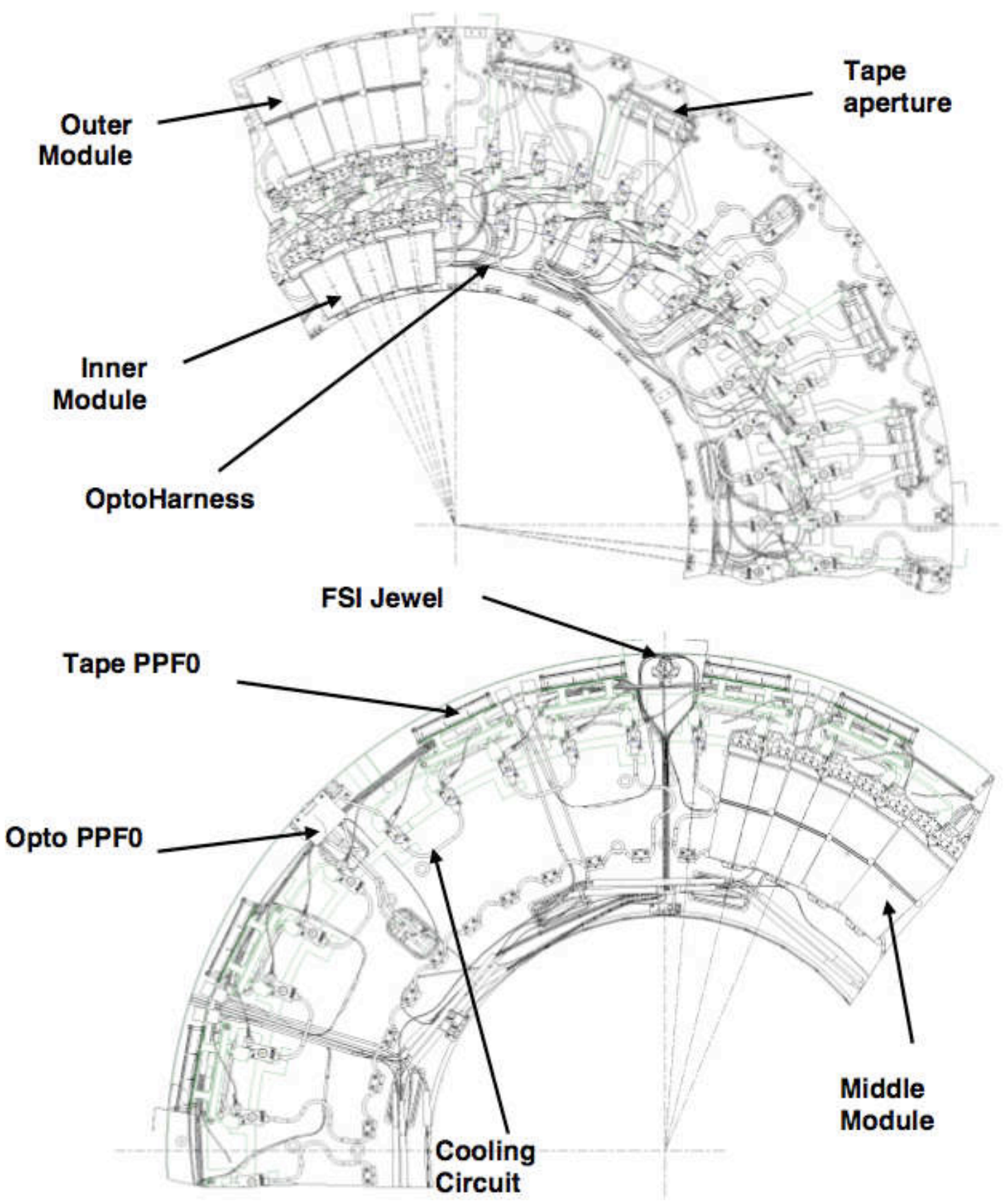

Figure 7. Services for disk 6 front (top) and rear (bottom).

block by a PEEK split separating two sections of carbon-carbon (visible in figure 9): the larger section of the block being available to cool the hybrid, while the smaller section is in contact with the "spine" (see figure 3) supporting the silicon [4]. The plane of high thermal conductivity of the carbon-carbon is chosen to be parallel to the split. Thermal contact is ensured by pressure from 

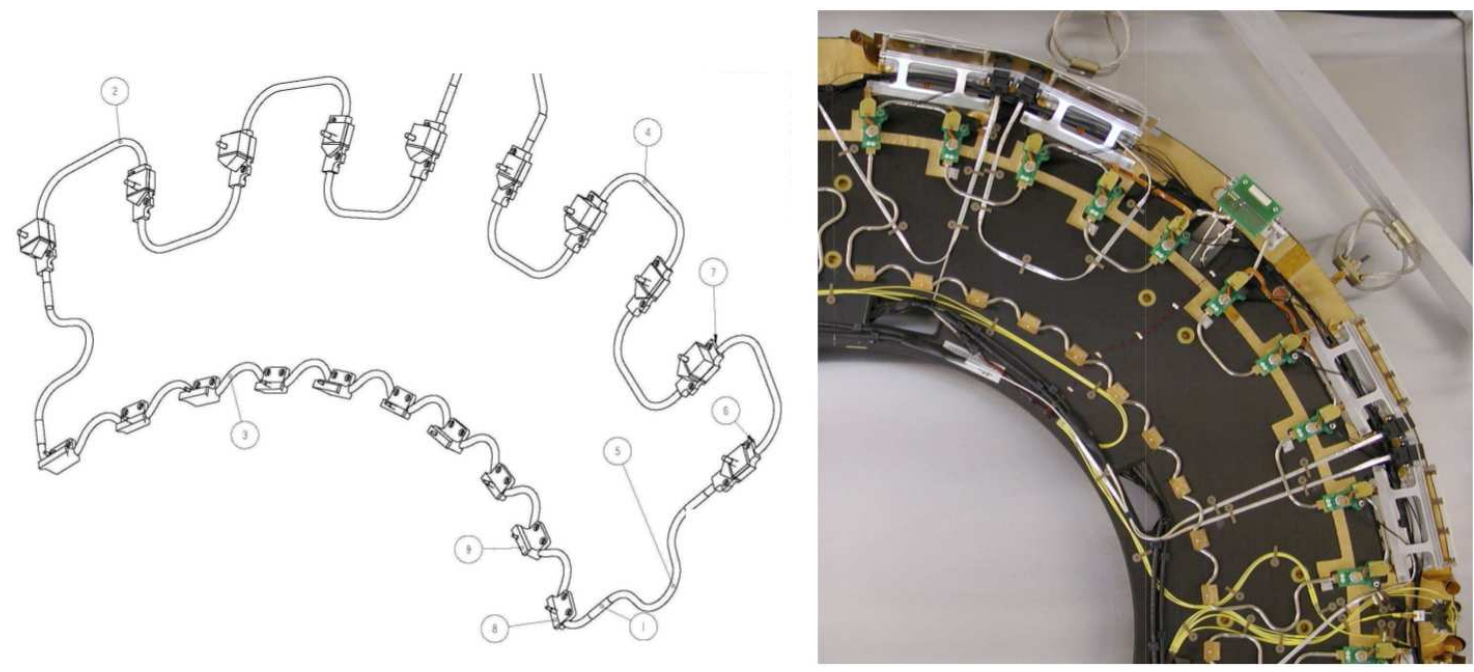

Figure 8. Cooling circuit for middle modules: design (left) and realisation on a disk (right).
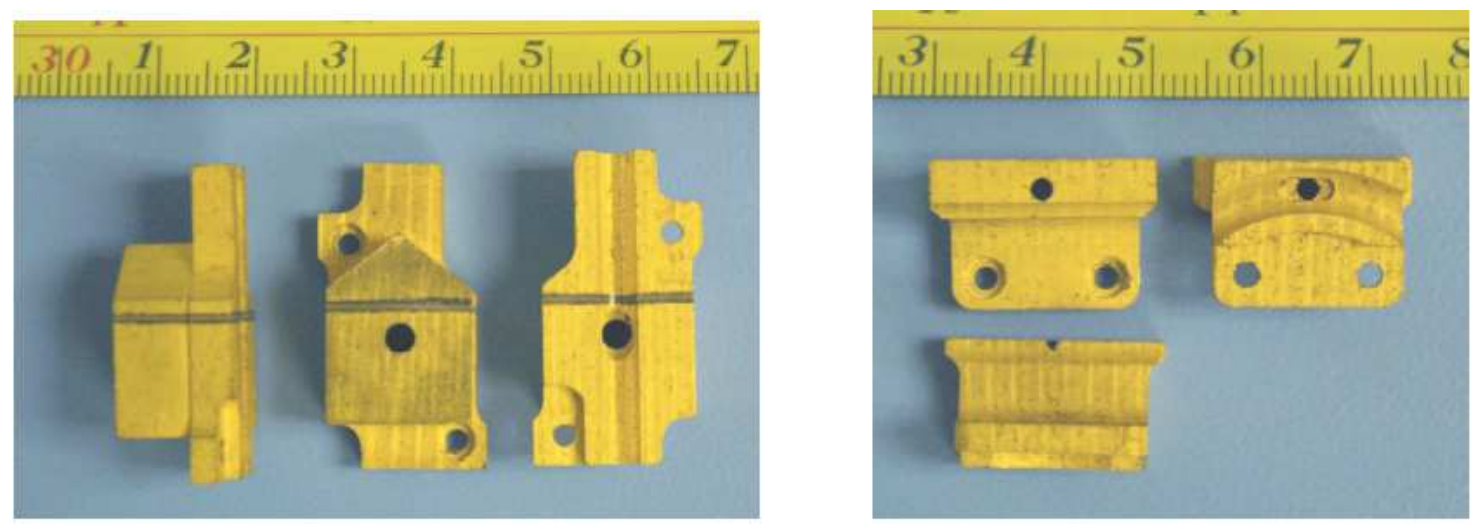

Figure 9. Cooling blocks: main (left) and secondary (right).

the module nut and a controlled layer of thermal grease ${ }^{15} 20 \mu \mathrm{m}$ thick. To ensure that the modules do not violate their envelope, potentially risking mechanical contact or electrical discharges with neighbours, and to avoid distorting the modules, the blocks were required to have a height variation of no more than $20 \mu \mathrm{m}$ and the height variation between the blocks at either end of a module should be no more than $100 \mu \mathrm{m}$.

The capillaries carrying the coolant to the disks (each associated with a single circuit) terminate at the cooling PPF0. However, the exhausts from all the circuits in one quadrant of a disk merge at PPF0, implying that a significant leak in a single circuit could cause the cooling for up to 33 modules to be lost. Both the inlets and exhausts are connected using aluminium seals. ${ }^{16}$ These are small rings with a metal jacket containing a spring. The seals are intended to be compressed in such a way that the metal jacket conforms to the surfaces of the housing, while the spring keeps the jacket pressed against the housing despite any movement due to thermal effects.

\footnotetext{
${ }^{15}$ DC340, manufactured by Dow Corning.

${ }^{16}$ Helicoflex $($ HN120 and HN100 seals, manufactured by Garlock Sealing Technologies.
} 
Initial FEA calculations [27] using CFX [28] indicated that in the gas between the disks, convection would give a temperature difference between the top and bottom of up to $14^{\circ} \mathrm{C}$. The simulations were broadly confirmed by a mock-up [29] which found significant heat-flows into the outer and middle module rings at the top of the end-cap and into the inner modules just below the ITE. This convective heat load on the front face of the modules, plus a load on the back face from the power tapes, plus a factor to account for uncertainties, amounted to $0.8 \mathrm{~W}$ for middle and outer modules and $0.4 \mathrm{~W}$ for inner modules. The modules have been designed to tolerate these loads from their environment without over heating.

\subsubsection{Prototyping}

The original intention had been to manufacture aluminium cooling circuits with aluminium cooling blocks. Because of concerns about corrosion, this solution was dropped and replaced with $\mathrm{Cu}-\mathrm{Ni}$. Because the radiation length of $\mathrm{Cu}-\mathrm{Ni}$ is much higher, a reduced wall thickness of $70 \mu \mathrm{m}$ was chosen to minimise material effects. Unfortunately the $\mathrm{Cu}-\mathrm{Ni}$ proved significantly harder to bend into "wiggles". The move to carbon-carbon cooling blocks benefited from a factor of three increase in radiation length of the material, but due to the difficulty of machining complicated shapes, the actual improvement in material was more like a factor of two. This partially compensated for the increased material coming from the $\mathrm{Cu}-\mathrm{Ni}$ pipes.

Several prototype cooling blocks were tested with $\mathrm{C}_{3} \mathrm{~F}_{8}$ evaporative coolant operating around $-18^{\circ} \mathrm{C}$. It was found that the dominant thermal impedance was between the pipe wall and the coolant itself. This was determined by measuring the heat transfer coefficient (HTC) for some simplified blocks. The HTC is a function of the coolant flow rate, vapour fraction and power density. It was found that in the expected regime, with a flow rate around $2 \mathrm{~g} / \mathrm{s}$ and vapour fractions in the range $10 \%$ to $85 \%$, the $\mathrm{HTC}$ is mainly a function of power density, $\mathrm{P}\left(\mathrm{W} / \mathrm{cm}^{2}\right)$, and can be conservatively represented by $H T C=330 \times P+1800\left(\mathrm{~W} / \mathrm{K} / \mathrm{m}^{2}\right)$. The result for the final block design with a $1 \mathrm{~mm}$ PEEK split was that at maximum module power, the detector and hybrid halves of the block ran at 8 and $13^{\circ} \mathrm{C}$ respectively above the coolant temperature. Finally, an irradiated inner module was tested on a prototype of the final block design and was stable against thermal runaway up to a coolant temperature of $-5^{\circ} \mathrm{C}$.

The original design for the cooling connections at PPF0 involved aluminium seals. These were subjected to a qualification procedure comparable to that described in section 2.2.1.3 and the seals were accepted on the basis of testing 10 of them and all 10 passing. To avoid possible corrosion problems associated with moisture, copper seals of a comparable design were considered. This solution was abandoned in the light of difficulties in compressing the stiffer seals so as to cause the rims of the housings to come together forming a stable connection. The requirements of low-mass connections and the limited space available makes it difficult to have housings capable of supplying substantial compression forces.

\subsubsection{Manufacture}

The cooling circuits were made in several parts: in particular the sections for the main and secondary cooling were manufactured separately and denoted by the "main" and "mini" wiggle pipes respectively. Bending the $\mathrm{Cu}-\mathrm{Ni}$ tube to form wiggles of the required bend-radius proved to be difficult because of the tendency of the tube to either crease on the inner side of the bend or rip on 
the outer side. In the case of the mini wiggles, the design called for a bend-radius of $4 \times$ diameter. Tests were made using fillers consisting of ice, "sand" (very small glass beads) and low-melting-

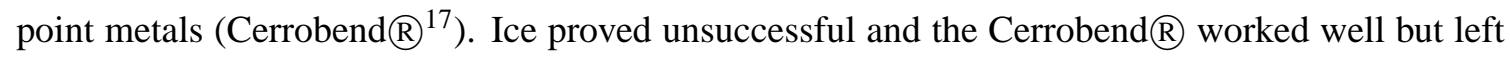
residues inside the pipe, giving concern that the material might flake off and subsequently cause blocks in the cooling system. After many trails, the best results were obtained by bending the main wiggles from fully annealed tube with a sand filler ${ }^{18}$ and the mini wiggles from half-hard tube in air. ${ }^{19}$ Resulting from the bending, the typical reductions in cross-sectional area were $12 \%$ and $4 \%$ respectively.

Cu-Ni tubes were supplied by High Tech Tubes Ltd. and Uniform Tubes Inc. (UTI). The tubes were fully annealed to start with, and then hardened as required by re-drawing from the fully annealed condition. Since it was difficult to harden or soften the pipe reliably, care had to be taken that none of the processes changed the pipe temper: this required tests before and after every thermal step in the process to ensure temper was maintained. It was found that cryogenic thermal shock and soft solder temperatures had no perceivable effect on the temper. Once bent, the pipes were stress-relieved in an oven.

The $70 \mu \mathrm{m}$ wall thickness was selected as the minimum wall thickness to have sufficiently many grains to ensure integrity at all levels of temper. This was measured to be as little as four grains in one fully annealed sample; however, even in this state, the pipe retained its leak tightness. A more significant cause of leaks was found to be inclusions in the material. To identify these, the pipes were thermally shocked several times and then leak tested. This reduced the yield to about 95\%. The pipes were leak tested before and after bending and no leaks were found due to the bending process; this meant that the only possible source of leaks post pipe QA was in the solder joints between pipes.

Due to small differences associated with the stereo module geometry, some portions of the circuit were different for the different orientations. The completed sections of pipes corresponding to the main cooling blocks were checked in a go-no-go jig with tolerances of $\pm 100 \mu \mathrm{m}$ to ensure that the stresses on the disk would not be too great. Pipes which did not drop naturally into the jig were adjusted if possible to ensure conformance.

The carbon-carbon blocks were difficult to machine - the material has an appearance and texture comparable to balsa-wood. Since the blocks are critical to the location of the silicon modules, a 10\% sample was measured and found to be well within specification, namely the upper and lower surfaces should be parallel to $10 \mu \mathrm{m}$ and the height within $20 \mu \mathrm{m}$ of the nominal value. To prevent the absorption of the thermal grease, the blocks were coated with a $1 \mu \mathrm{m}$ layer of gold on top of $12 \mu \mathrm{m}$ of copper. The module location pin was inserted through a hole, located by shoulders on the base of the pin and fixed in place by solder. The blocks were located relative to the pipe by an accurate jig and soldered in place. Different sections of the pipe were connected by soldering them together with enlarged sleeves to create complete circuits.

Once the circuits were assembled, they were then cleaned and tested, avoiding contact with water at all times. The first step was to clean the internal surface of the pipe by flushing and

\footnotetext{
${ }^{17}$ Manufactured by Cerro Metal Products, also knows as Wood's metal.

${ }^{18}$ Undertaken by Newtown Engineering.

${ }^{19}$ Undertaken at the Daresbury Laboratory (part of STFC).
} 
soaking the system overnight using a commercial cleaning fluid ${ }^{20}$ with a dedicated cleaning rig with removable filters and a fume extraction system. Any leaks which developed, due to the removal of flux by the liquid, meant that the system had to be drained and dried with nitrogen before any repairs could be undertaken. After successful cleaning (established by visual examination of the removable filter), the pipes were flushed with nitrogen to dry the surfaces.

The circuits were then loosely mounted on the disks where they were pressure tested to 25 bar, ${ }^{21}$ with helium. With the disk inside a sealed enclosure, a helium leak-detector was used to sample the volume and check for leaks. The pipe was then vented and connected to a vacuum pumping system to ensure that a pre-defined vacuum pressure could be achieved. Helium was then introduced into the sealed enclosure and the helium leak-rate checked. It was not considered necessary to thermally cycle the circuit, since this was done when testing the modules (see section 3.2).

\subsubsection{Other services}

\subsubsection{Power tapes}

Power is supplied to the modules and opto-packages via "wiggly" power tapes [30]. The design was based on double-sided $\mathrm{Cu}$-polyimide flexible circuits for the low-current power lines, temperature monitoring and control lines, and copper-clad aluminium (CCA) twisted pairs for the main current-carrying conductors. For the Cu-polyimide tapes, the copper is $18 \mu \mathrm{m}$ thick and the polyimide is $25 \mu \mathrm{m}$. The lines are $150 \mu \mathrm{m}$ wide with a $150 \mu \mathrm{m}$ gap between traces, except for the high-voltage (HV) line which has a separation of $800 \mu \mathrm{m}$ to the nearest line in order to minimise leakage currents. ${ }^{22}$ In addition, a polyimide cover-layer was used to provide isolation. In the region near the connectors where there was no cover-layer, the separation between the HV line and the neighbour lines was increased to $2.5 \mathrm{~mm}$. The first tapes were produced with a $50 \mu \mathrm{m}$ thick photo-imageable cover-layer. However, the resulting tapes were found to be rather fragile; so for the rest of the production, a conventional polyimide cover-layer was used. The low-voltage (LV) digital and analogue power for the module (higher current) is carried by twisted pairs made of $0.55 \mathrm{~mm}$ diameter CCA with an insulating lacquer coating and with 2 turns per inch. The wire composition by volume is $90 \%$ aluminium and $10 \%$ copper. The CCA twisted pairs were attached to the $\mathrm{Cu}$-polyimide tapes with sticky polyimide tape. ${ }^{23}$ As the CCA is much more rigid than the $\mathrm{Cu}$-polyimide, this tended to cause breaks in the narrow copper traces when the tapes were bent into the required 3D shape. In retrospect, a more robust design would have kept the CCA twisted pairs and $\mathrm{Cu}$-polyimide tape mechanically separated.

To reduce the numbers of flavours of tapes, power for up to three modules is incorporated in a single tape - the three modules sharing a common tape will have similar azimuthal position but not necessarily be in the same rings. The ends were rigidised for stress-relief and terminated with connectors $^{24}$ for connection to the module hybrids at one end and the PPF0 at the other. The CCA twisted pairs were soldered to pads on the tape near the connectors. Due to the mismatch in the

\footnotetext{
${ }^{20}$ Swansolv ionic cleaner, manufactured by Swan Tek.

${ }^{21}$ The operating pressure is 7 bar. The maximum overpressure expected in a fault condition is 16 bar; so a safety factor of $50 \%$ has been added to this for testing.

${ }^{22}$ Specification IPCC 2221.

${ }^{23}$ The sticky tape was purely an assembly aid. After assembly, the power tapes are held in place by the cooling circuits.

${ }^{24}$ Samtec FTM-114-03-L-DV and CLP-115-02-L-DV.
} 


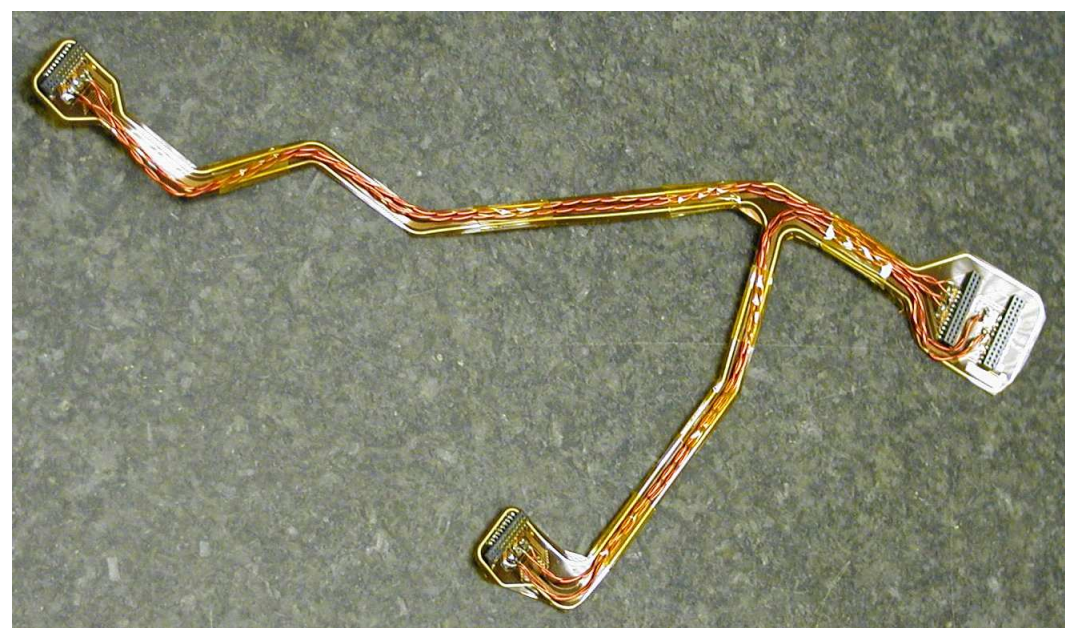

Figure 10. A typical "wiggly" power tape to power two modules. The module connectors are at left and middle; the PPFO PCB is at right. The LV twisted pairs can be seen above the polyimide flex circuit.

number of modules in the different rings causing the modules not to line up in azimuth and the absence of inner modules on four disks, the only replication which occurs is by quadrant and 21 different "flavours" of tapes are required. A typical tape is shown in figure 10 .

\subsubsection{OptoHarnesses}

Data from the detector is transferred to the off-detector electronics by optical fibres. Timing, trigger and control (TTC) data are also transferred from the off-detector electronics to the detector by optical fibres. Two data and one TTC fibre serve one detector module. The data and TTC fibres from between 4 and 6 modules are spliced into 12-way ribbon fibres to form a fibre harness. To allow for broken fibres, both for readout and TTC, redundancy links are incorporated into the design. More information on the fibre harnesses is available in [31]. One critical issue for the harnesses associated with the engineering was that the fibres have to be light-tight in order to avoid generating noise in the silicon detectors. This meant that the individual fibres were in $0.9 \mathrm{~mm}$ OD black furcation tubing and the ribbons were wrapped with aluminium foil. Unfortunately the black furcation tubing was difficult to see next to the carbon fibre. ${ }^{25}$ Because the fibre routing was complicated, it had to be performed by hand. Very thin furcation tubing was required because of the tight clearances, consequently it provided little protection for the delicate fibres and there were several fibres damaged during the assembly which required the harnesses to be removed from the disk.

\subsubsection{FSI (Frequency scanning interferometry)}

The ATLAS FSI alignment system is documented elsewhere [11]. Each SCT end-cap is equipped with 165 grid line interferometers (GLI's) in the disk planes and connecting adjacent disks. Altogether, they make an over-constrained grid. Details of the end-cap layout can be found in [13]. The length of the GLI's can be measured to better than $1 \mu \mathrm{m}$ by virtue of interferometry with variable wavelength light. Variations in the measured GLI lengths indicate distortions of the disks or

\footnotetext{
${ }^{25}$ Yellow furcation tubing had been tested; however, this was transparent to infra-red light and therefore useless.
} 

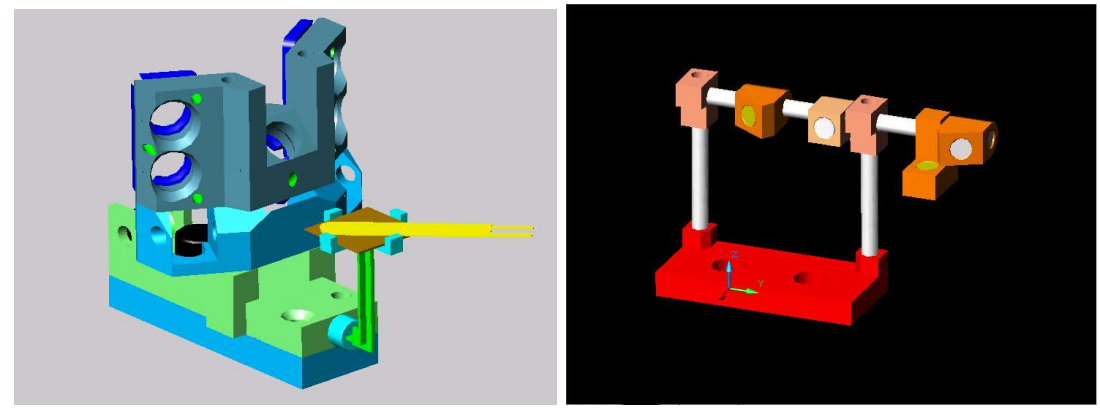

Figure 11. Typical FSI jewel (left) and reflector (right). Note the thermistor (yellow) mounted on its own stand, to the right of the jewel.

relative movements of the disks. Only relative motion (potentially caused by thermal effects or the relaxation of the support structures) can be determined, since the absolute positions of end-points of the light paths are not known accurately. The FSI system provides information on correlated displacements of modules and hence is very important for determining large-scale deformations of the detector - this is highly complementary to the information provided by the offline alignment using reconstructed charged tracks.

Each GLI is formed by a pair of a "quill" and a retro-reflector. Quills are assemblies of a delivery and a return single-mode opto-fibres attached to a glass beam-splitter. Each quill emits a cone of infra-red light (opening angle approximately $2.5^{\circ}$ ) directed towards the retro-reflector. The reflectors are made from small aluminium cylinders, $2 \mathrm{~mm}$ in diameter, into whose surface was punched an accurate cube corner; each cylinder was subsequently gold-plated. The reflected light is received by the same quill into its return fibre, detected by an avalanche photo-diode (APD) at the other end and subsequently subjected to interferometric analysis with respect to a stable reference length. For the method to be accurate, it is important to monitor the temperature of the ambient gas in the vicinity of the light-path and to ensure a stable gas composition since these affect the refractive index of the gas and hence the optical path length.

Grid nodes which contain end-points of several GLI's are made into so-called "jewels" which hold individual quills or retro-reflectors. The grid is complicated and the location of the nodes varies from disk to disk to adapt to changing disk spacing. Typically there are three jewels at the outer disk radius and three or six on the inner radius. One side of the disk is equipped with jewels holding mostly quills while the other side contains jewels with retro-reflectors only. GLI's are formed between the outer points, between the outer and the inner points and between the outer points and the inner points of the neighbouring disk. The jewels are predominately made from glass-filled Torlon $\AA$ which is a plastic stable to moisture and temperature variations. To have lines of sight above the rest of the services, the jewels are supported on a pair of thin quartz rods and mounted on a PEEK base which is fixed to the disk. The jewels need to be mounted with a spatial precision of better than $1 \mathrm{~mm}$ and angular precision of better than $1^{\circ}$. Example jewel assemblies are shown in figure 11 .

The optical ribbons from individual jewels run to the dedicated splice boxes attached to the electromagnetic calorimeter cryostat with no break at PPF0, in order to minimise light loss and 
back reflections. There, they are spliced into multi-ribbon cables which route the light to the readout crates.

\subsubsection{Detector Control System (DCS)}

Sensors for the DCS [32] were placed all over the disks (see [26] for more details). Up to 30 thermistors were placed on each disk: on the exhausts of the cooling circuits, on some locations in the middle of the circuits, on the CFRP disks and on the FSI Jewels (see figure 11). These consist of flat $1 \mathrm{k} \Omega$ thermistors encased in polyimide and read out with twisted pairs. Each disk was instrumented with a single humidity sensor ${ }^{26}$ consisting of a three-wire package in a carbon-fibre housing [33].

\subsubsection{Patch Panels (PPF0)}

There are patch panels for most of the services at the outer radius of each disk on the rear side.

There are four cooling patch panels per disk consisting of the inlet and exhaust connections, described in section 2.2.1.2, along with the associated support bracket.

There are 16 electrical patch panels per disk. These consist of the rigidised ends of the "wiggly" power tapes held in brackets. Each patch panel serves up to 9 modules via 3 3-way tapes (note: not all positions are used). There are 8 optical patch panels per disk and 4 for DCS.

The patch panels for disk 9 are on the front side, since the disk has been rotated back-to-front (see section 1.2). This requires some modified circuitry on the ends of the "wiggly" power tapes to cope with the swap to the order of the power lines.

\subsubsection{Miscellany}

To ensure that the disks and associated services are grounded, aluminium-polyimide foils are placed on the disks. The foil is plated to allow good solder connections to be made: polyimide $(25 \mu \mathrm{m})$, glue $(25 \mu \mathrm{m}), \mathrm{Al}(50 \mu \mathrm{m}), \mathrm{Ni}(12 \mu \mathrm{m}), \mathrm{Au}(2 \mu \mathrm{m})$. There are two foils; one on the front and one on the back of the disk which cover approximately $6 \%$ and $19 \%$ of the surface area respectively - see figure 12. The foils are connected to the ground pads on the CFRP disk and to the cooling circuits by soldered tabs. In turn, the cooling circuits are connected to the ATLAS ground via connections to the ground sheet on the support cylinder (see section 4.2).

\subsubsection{Services assembly and testing}

The assembly of all the services to each CFRP disk took place at the RAL and NIKHEF. Each disk required 3-4 months, including testing. Services were added one layer at a time: grounding foils, wiggly power tapes and optoharnesses. These were tested before they were trapped by the rest of the services: cooling circuits, DCS and FSI jewels. Extensive testing was undertaken of the power tapes, the optoharnesses [31] and the cooling circuits. Any significant damage of the tapes or harnesses required that the cooling circuits were removed to allow replacement - this was time consuming. The result was that every completed disk was fully functional.

After this, the cooling blocks, which until this point were only loosely placed, were positioned accurately. By design the screw holes in the blocks were $300 \mu \mathrm{m}$ oversized allowing the blocks

\footnotetext{
${ }^{26}$ Xeritron ${ }^{\mathrm{TM}} \mathrm{XN} 1018$ sensors, manufactured by Hygrometrix Inc..
} 


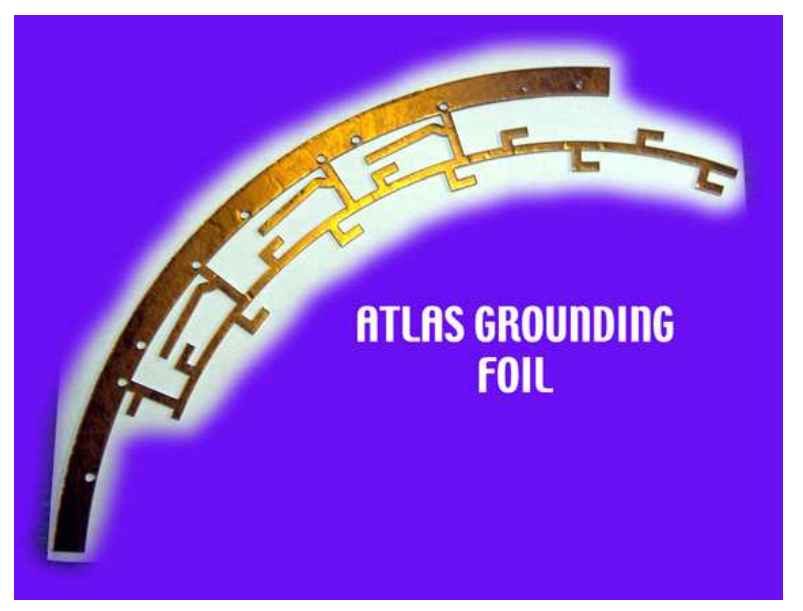

Figure 12. Front ground foil for a disk.

to be moved and located accurately. For EC-C, the disk was placed on a digital rotary table and centralised using the two central alignment holes in the disk by viewing through an optical system. Custom made targets were placed over the pins in the blocks and the blocks were adjusted by hand ${ }^{27}$ to their defined positions, again by viewing under the same optical system. Once located, the block retaining screws were tightened to a pre-defined torque. At the same time, the base-pads for the FSI jewels and reflectors were placed. For EC-A, a precision-machined plate was used. The tolerances for the pin placement to ensure that all the modules overlapped without gaps were: 37 , 60 and $190 \mu \mathrm{m}$ (see table 2) for the outer, middle and inner rings respectively.

The final FSI components, which were above the silicon modules on the front of the disks, could only be placed once the module mounting (see section 3 ) was completed. It was not possible to test the FSI components, but from careful inspection of the fibres and jewels, there are no known problems.

\footnotetext{
${ }^{27}$ The design of the "wiggly" circuits permitted easy movement of the blocks of the order of $1 \mathrm{~mm}$.
} 


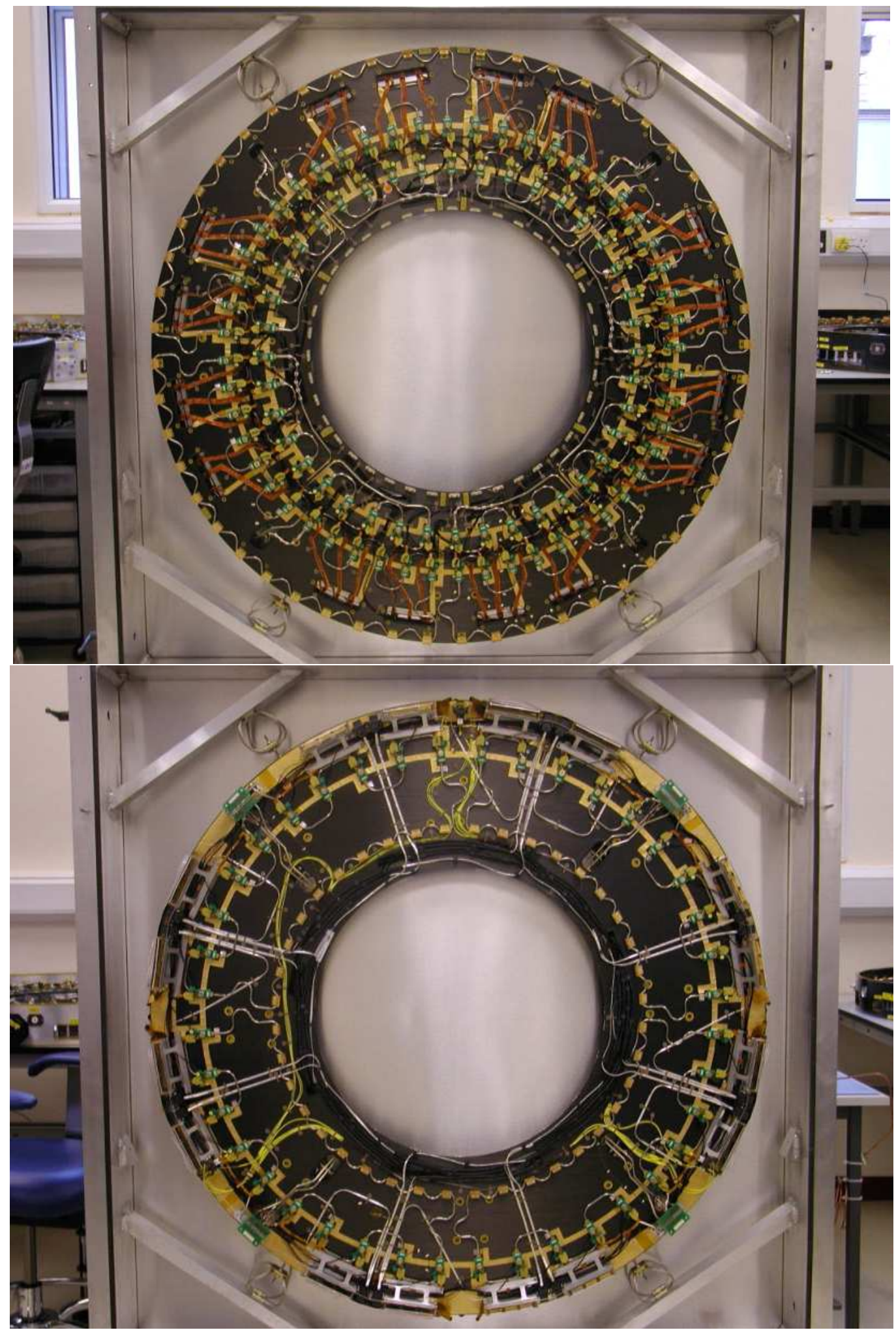

Figure 13. Disk 6C with services, ready for modules: front (top) and rear (bottom). 


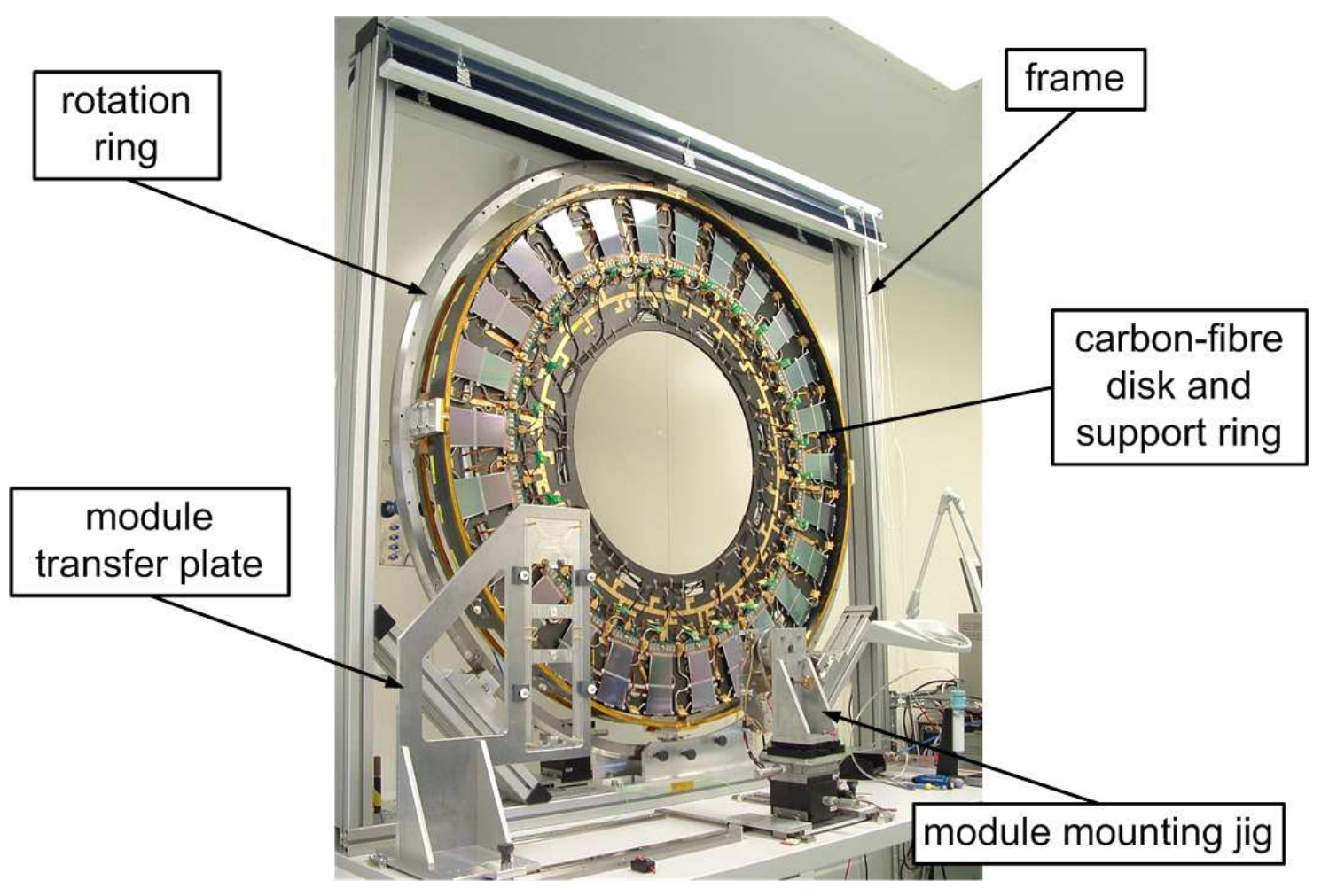

Figure 14. Tooling for module mounting.

\section{Module mounting}

\subsection{Module mounting}

Modules [4] from the module construction sites were delivered to The University of Liverpool and NIKHEF as needed for mounting on to the disks. At both sites, modules were mounted on to disks using similar techniques designed primarily to accomplish the task with minimal risk to the modules. This required the design and manufacture of tooling to allow the disk to rotate and modules to be mounted onto the cooling blocks, as well as the development of procedures to dispense thermal grease, fasten down the modules and attach the services. The modules are staggered in height on the disk to ensure that there is an overlap between each module and its neighbour. At the start of the build, all lower modules of all ring-types would be mounted in groups of about six; the disk was tested and then module mounting resumed with the addition of the upper modules, after which the disk was tested again. However, very few defective modules were found in testing, and the later disks were built without the intermediate tests.

The basic concept of the tooling is shown in figure 14. The disk was mounted on a rotating ring mounted in a frame which allowed the disk to be positioned at precisely the right angle so that the mounting points for all modules could be brought to exactly the same position relative to the module mounting jigs. Modules were then mounted on the module transfer plate and extracted from their frames before being located on to the cooling block pins on the disk using the module mounting jig. 


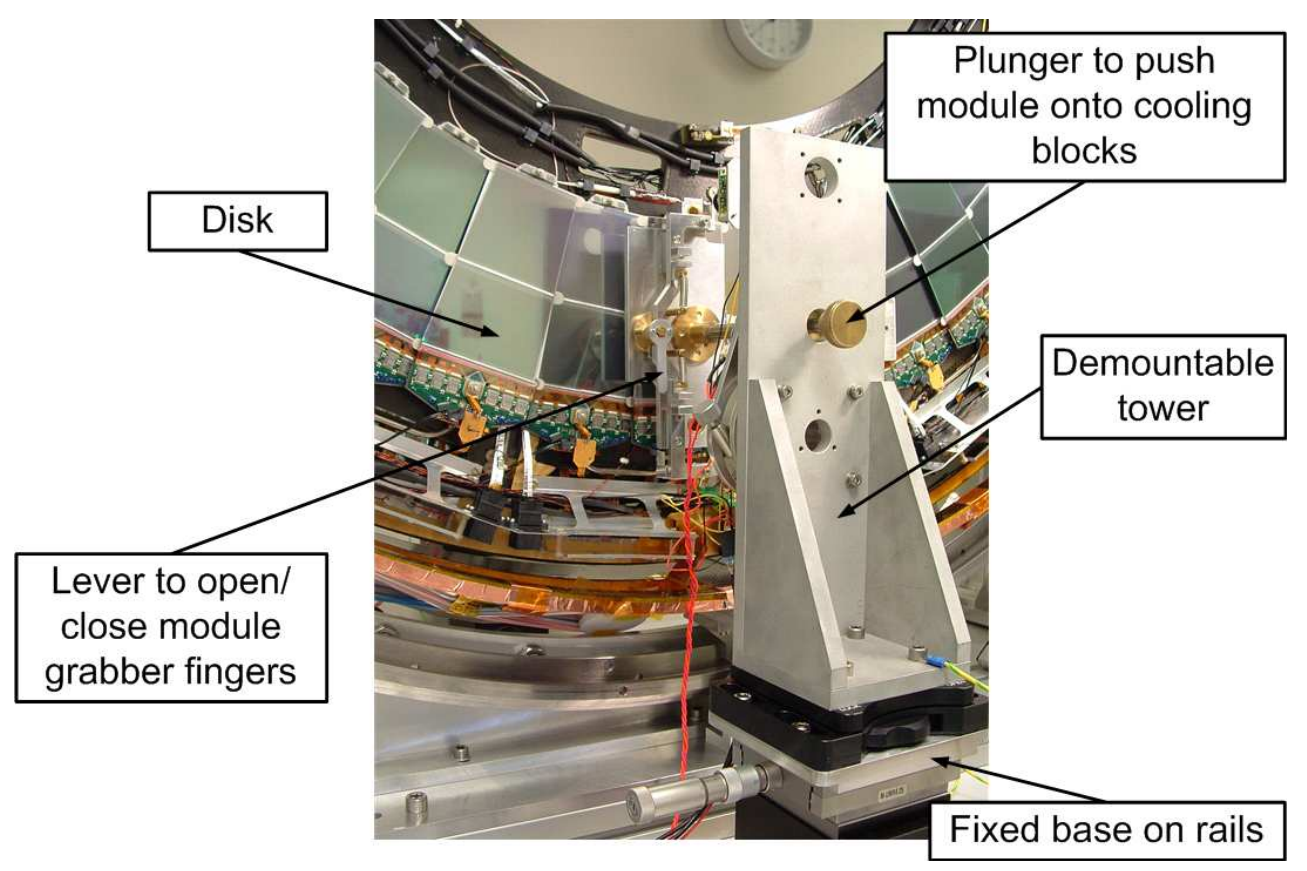

Figure 15. Module mounting jig.

The module mounting jigs consisted of a base and an interchangeable tower, as shown in figure 15. The base was mounted on precision rails running perpendicular to the disk. Left-right and up-down translation stages were used to position the tower relative to the disk. The tower supported a module grabber which allowed the module's angle and position (in the direction of the rails supporting the base) to be adjusted. Miniature CCD cameras were used to view the relative positions of the module's precision mounting holes with the corresponding pins on the cooling blocks to allow the operator to correctly position the module before it was slid on using the grabber.

The thermal requirements dictate that the amount of thermal grease making the cooling contact between the modules and their cooling blocks be carefully controlled. This was achieved using a precision liquid dispenser and a syringe as shown in figure 16. Thermal grease ${ }^{28}$ was applied to the main-point blocks using a 10-dot pattern, whilst 6 dots were applied for the second-point blocks. The mass of grease applied was adjusted by varying the air pressure and air-pulse duration on the dispenser. The mass of grease dispensed was checked before a group of five or six modules was mounted by applying grease to the sample block. The typical grease coverage over a block was checked by mounting a clear plastic plate on the sample block and applying the torque used for module mounting.

Module mounting began with the preparation of a list of modules for each ring type selected using the performance parameters obtained during module testing at the assembly sites. The selected modules were moved to storage near the module mounting station. Typically, module mounting proceeded by ring type, block height and quadrant. For example the five lower modules in the top right quadrant (TR) of the middle ring would be mounted sequentially. Using the preliminary

\footnotetext{
${ }^{28}$ DC 340, manufactured by Dow Corning.
} 


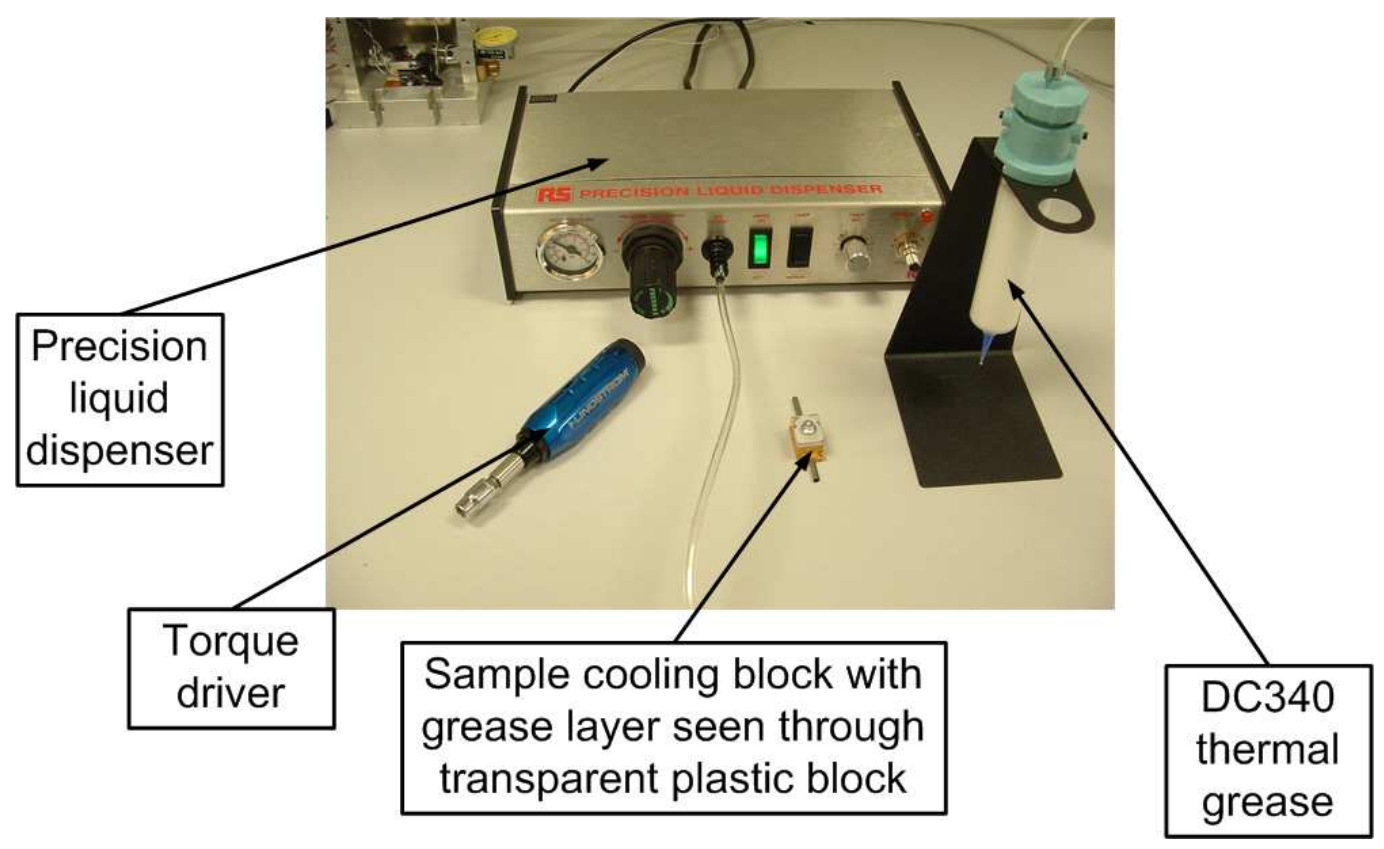

Figure 16. Tooling for grease application to module cooling blocks.

module lists, the five required modules were identified and laid out in mounting order. Then, the grease dispenser was set up, adjusting the parameters to get the right amount of grease, and grease was dispensed on each of the five pairs of cooling blocks (main and secondary). The disk was positioned using the indexing holes in the rotation ring such that the blocks for the first module were at the bottom.

The first module, still held in its frame, was then mounted on the module transfer plate. The plate was slid and locked in a position immediately in front of the module mounting jig. The jig was then slid forward and locked in position. The lever to open the module grabber jaws was activated and, using the CCD cameras for feedback, the position of the grabber was adjusted using the translation and rotation stages and the grabber plunger. The grabber jaws were closed and a check was made to ensure that the module was being held properly. The module was then released from its frame, the module mounting jig was withdrawn and the module transfer plate slid back.

With the module now on the end of the grabber, the module mounting jig was advanced and positioned just in front of the disk. Again the position of the module was adjusted using the CCD image of the module mounting pins in the two cooling blocks as a reference. Once the module was correctly aligned, it was pushed onto the blocks using the plunger of the module grabber.

The module was released from the grabber and the module mounting jig was withdrawn. The module would remain in its proper position due to the slight stickiness of the thermal grease. The far-end nut was loaded into its driver. The far-end washer was located onto the pin using tweezers and the nut was screwed on until the module was just being held. This was repeated with the mainpoint pin but with the added complication of the "grounding finger" which was slid over the pin before the washer and nut. When both nuts were on their respective pins, a torque driver was used for the final tightening. 


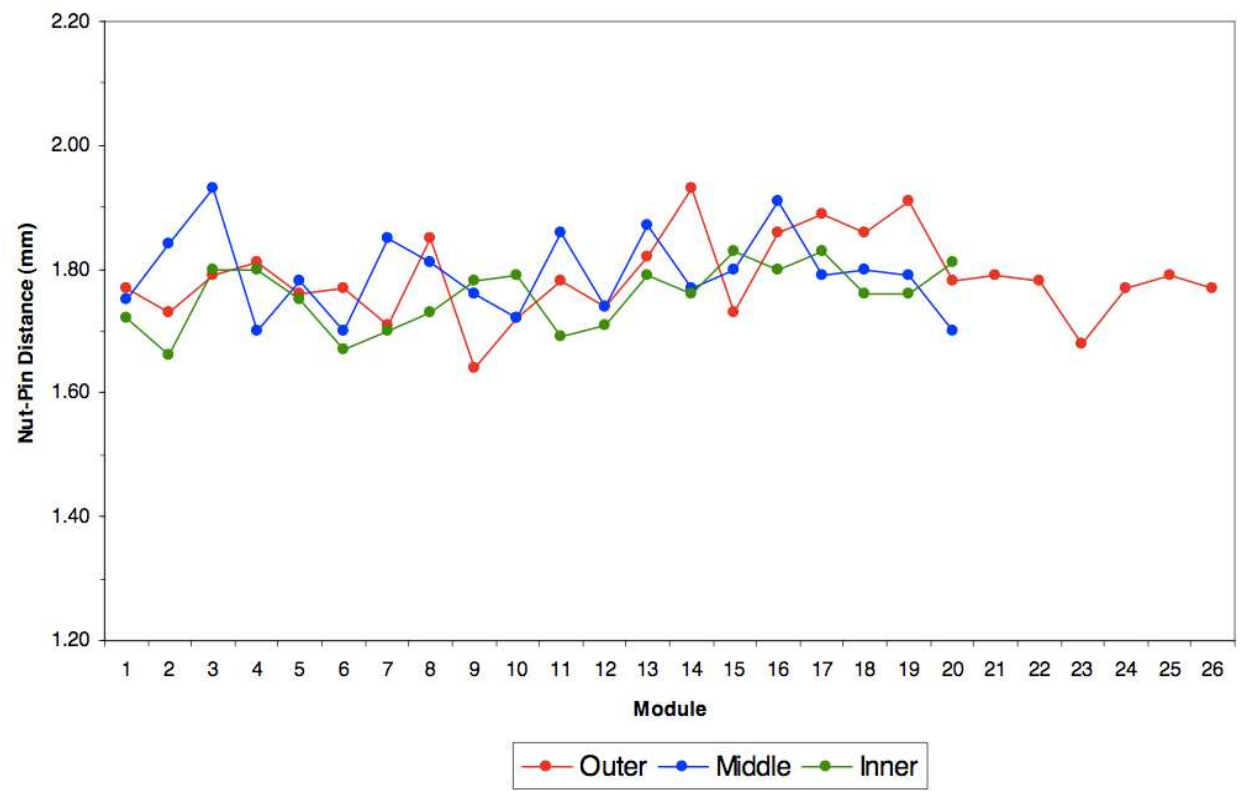

Figure 17. Distance from the top of the main-point nut to the top of the main-point pin — an example of 66 modules on disk 6C.

As a final check, the distance from the top of the main-point nut to the top of the main-point pin was measured. As an example, the graph in figure 17 shows the values obtained for the 66 lower modules on disk 6C. Any modules with distances greater than $1.9 \mathrm{~mm}$ were re-checked. Typical problems were associated with the washer or grounding finger catching in the threads of the pin causing the torque threshold to be reached before the module was properly secured.

The positions in the plane of all of the mounting pins relative to the reference holes in the disks were measured on a CMM with a precision of $10 \mu \mathrm{m}$ and incorporated into the alignment database in order to provide an initial estimate of the position of each module on a disk [34]. The resultant mean offset deduced for all modules was $56 \mu \mathrm{m}$ in $\mathrm{R} \phi$ with r.m.s.'s for EC-A and EC-C of $58 \mu \mathrm{m}$ and $53 \mu \mathrm{m}$ respectively. For the radial displacements, the corresponding numbers are $41 \mu \mathrm{m}, 38 \mu \mathrm{m}$ and $43 \mu \mathrm{m}$. The alignment accuracy which was achieved was comparable for all rings of modules; however the specifications are tightest for the inner rings where it was proposed that module pins should be placed to $37 \mu \mathrm{m}$ (see table 2). This specification was not met since there were often global rotations of all of the pin positions in a given ring. Since the specification is intended to ensure that there is a sufficiently large overlap between two neighbouring modules in a ring, a more useful requirement is that the overlap should not be reduced by more than $74 \mu \mathrm{m}$ (even then, this is not a hard limit, since the nominal overlaps are 0.4-1.4 mm). This was met in all cases except one overlap which was $76 \mu \mathrm{m}$. So in conclusion, the pin placement should be considered to be very satisfactory.

The mounting of modules at Liverpool began on September 2004 with disk 9C and the last module on disk 1C was mounted on July 2005. The graph in figure 18 shows the progress made. In NIKHEF, the mounting began on May 2005 and was completed on January 2006. A completed disk is show in figure 19. 


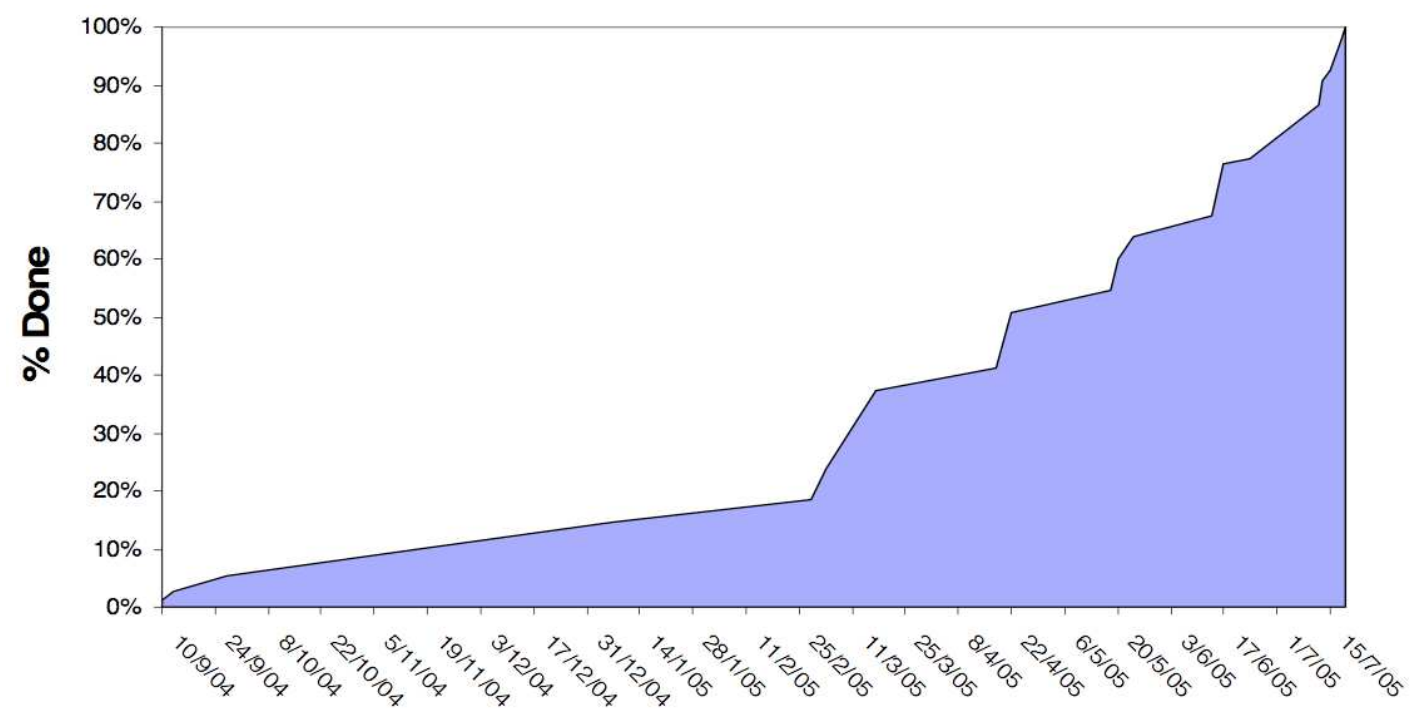

\section{Date}

Figure 18. Progress in mounting modules at Liverpool (EC-C).
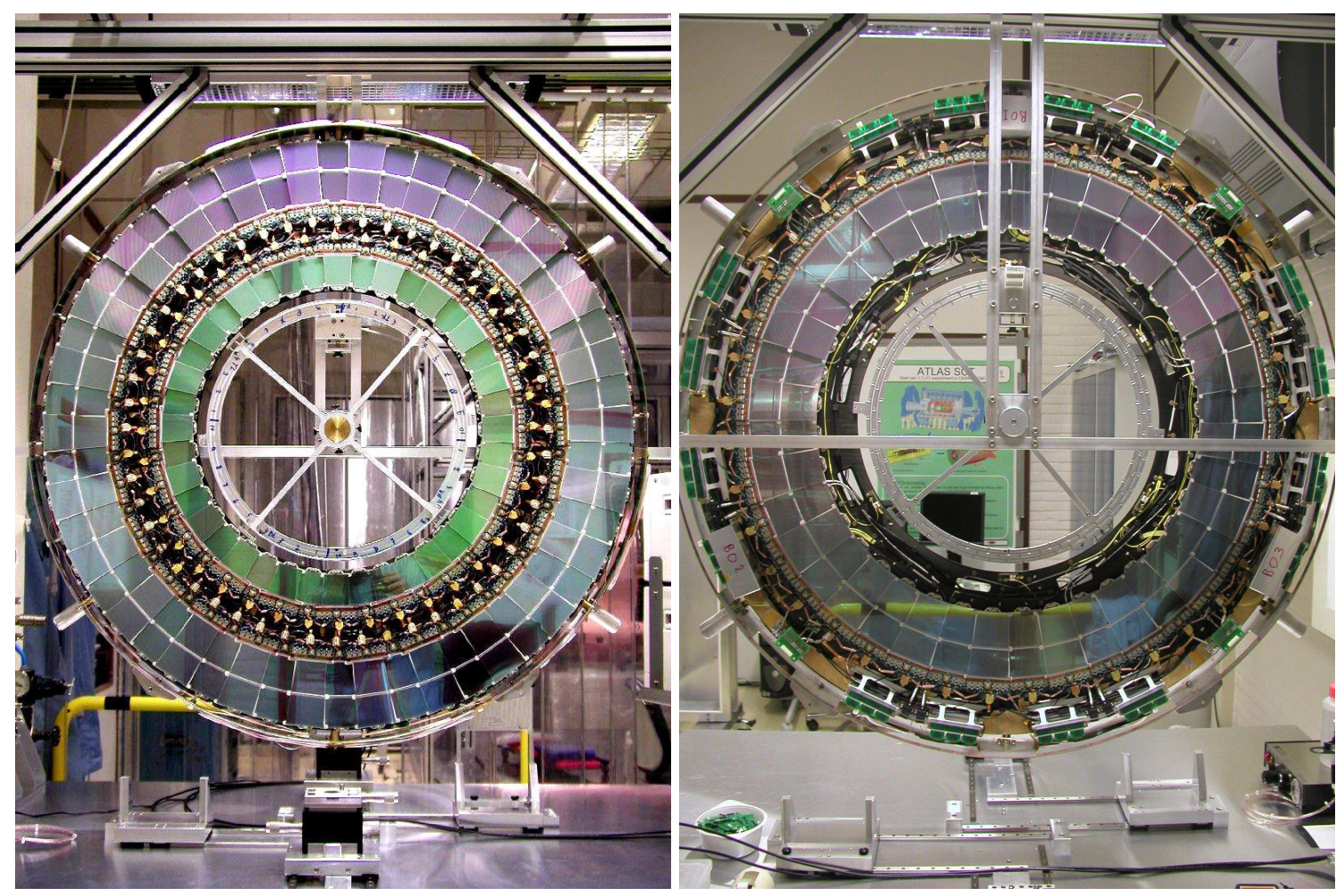

Figure 19. Completed discs: front, disk 6A (left) and rear disk 1A (right).

\subsection{Testing}

After the disks were assembled, they were electrically tested [35] to check that none of the modules 


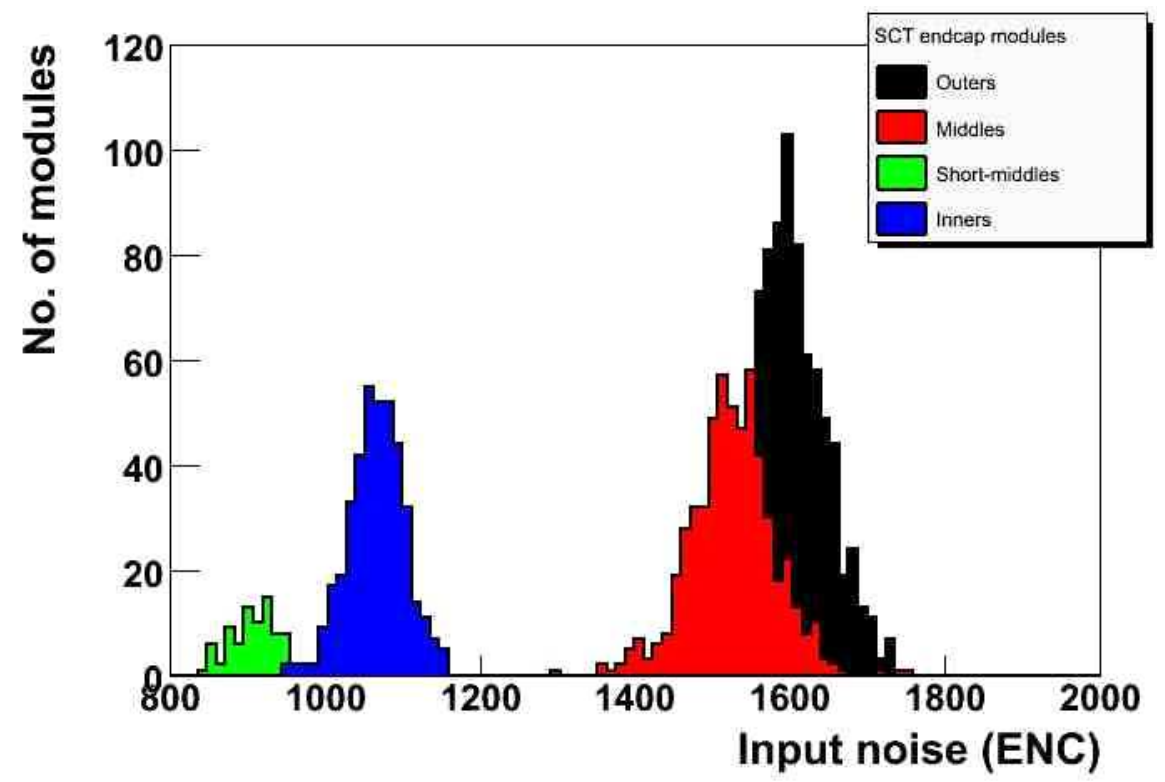

Figure 20. The input noise for each of the modules on both end-caps after module mounting.

had been damaged during the assembly procedure. The disks were placed inside a test-box which sat inside an environment chamber where the air humidity and temperature could be controlled. The dew-point in the chamber was kept around $-30^{\circ} \mathrm{C}$. The modules were cooled to their nominal operating temperature using the SCT evaporative cooling system for EC-C (for EC-A, modules were cooled to just below $0^{\circ} \mathrm{C}$ ). The modules were powered [36] using the custom-made SCT power-supply system and read out optically using the SCT ROD's (readout driver), BOC's (backof-crate card) and TIM (TTC interface module) [37, 38]. The maximum number of modules which could be readout simultaneously corresponded to one quadrant of a disk.

The measured input noise for each module type is shown in figure 20 and the average for each of the module types is shown in table 6. The numbers of problematic strips are shown in figure 21 . There are a total of 8000 problematic strips in the two end-caps (4040 in EC-C and 3960 in EC-A, including one dead chip) $-0.26 \%$ of the total number. The electronically dead strips are $79 \%$ of the problems, the rest correspond to noisy or unbonded strips. The average number of defects per module is 4.0 - well below the specification of a maximum of $15(1 \%)$. By iterating the processes of mounting and testing, no net additional defects were introduced during the assembly process.

\section{Support structures}

\subsection{Design}

The SCT end-cap support structures [39] support the disks within the inner detector. Each end-cap has a support cylinder, a front support panel, a rear support panel and an inner thermal enclosure (ITE) cylinder. The support cylinder locates and supports the disks, while the front and rear supports hold the cylinder on the TRT rails so that the SCT end-cap and TRT end-cap are coaxial (the 


\begin{tabular}{|l|l|l|}
\hline Module Type & \multicolumn{2}{|l|}{ Noise (ENC) } \\
\hline & EC-C & EC-A \\
\hline Outer & 1577 & 1605 \\
\hline Middle & 1530 & 1525 \\
\hline Short Middle & 922 & 914 \\
\hline Inner & 1074 & 1065 \\
\hline
\end{tabular}

Table 6. The average input noise corrected to $0^{\circ} \mathrm{C}$ for each module type.

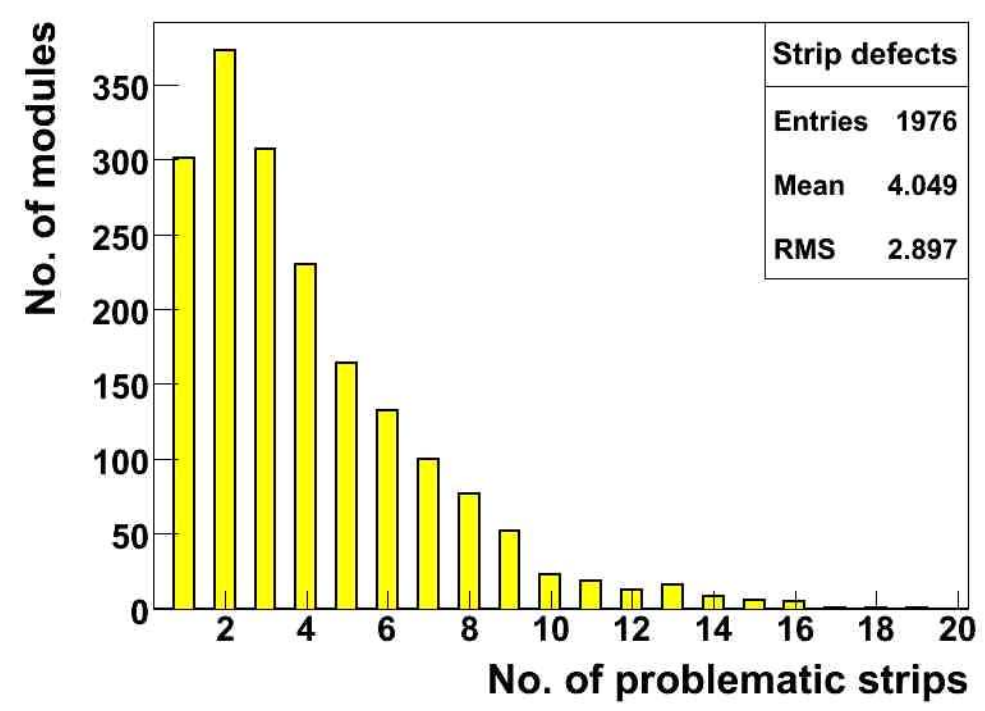

Figure 21. The number of modules having a given number of problematic strips, for both end-caps.

TRT is in turn supported by rails fixed to the inner bore of the cryostat). The support cylinder also carries services to and from the disks. The front supports, in-fill panels (part of the front support) and rear supports along with the ITE cylinder form essential components of the thermal enclosure, discussed in section 8 .

The support cylinder, front supports and rear supports are composite sandwich structures using CFRP facesheets. ${ }^{29}$ The core of the sandwich panels is a high-performance aramid/phenolic honeycomb core ${ }^{30}$ For the support cylinder, the facesheets and core were co-cured in an autoclave using an adhesive film. ${ }^{31}$ For the front and rear support, an epoxy film adhesive ${ }^{32}$ was used to bond

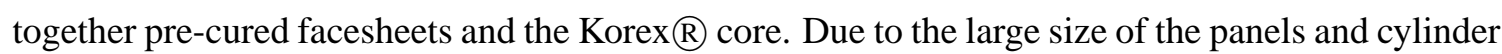
compared to the stock honeycomb sheets size, the honeycomb core was spliced together using an expanding syntactic film. ${ }^{33}$ All edges were sealed to prevent the ingress of moisture: CFRP close-

\footnotetext{
${ }^{29}$ YSH-50A graphite fibres in an RS-3 cyanate-ester resin matrix, manufactured by YLA Inc.

${ }^{30}$ Korex-5/32-2.4, manufactured by Hexcel Composites.

${ }^{31} \mathrm{RS} 4$ cyanate-ester adhesive film, manufactured by YLA Inc..

${ }^{32}$ FM73U, manufactured by Cytec Engineered Materials.

${ }^{33}$ SynSpand $9899.1 \mathrm{CF}$, manufactured by Loctite Aerospace.
} 


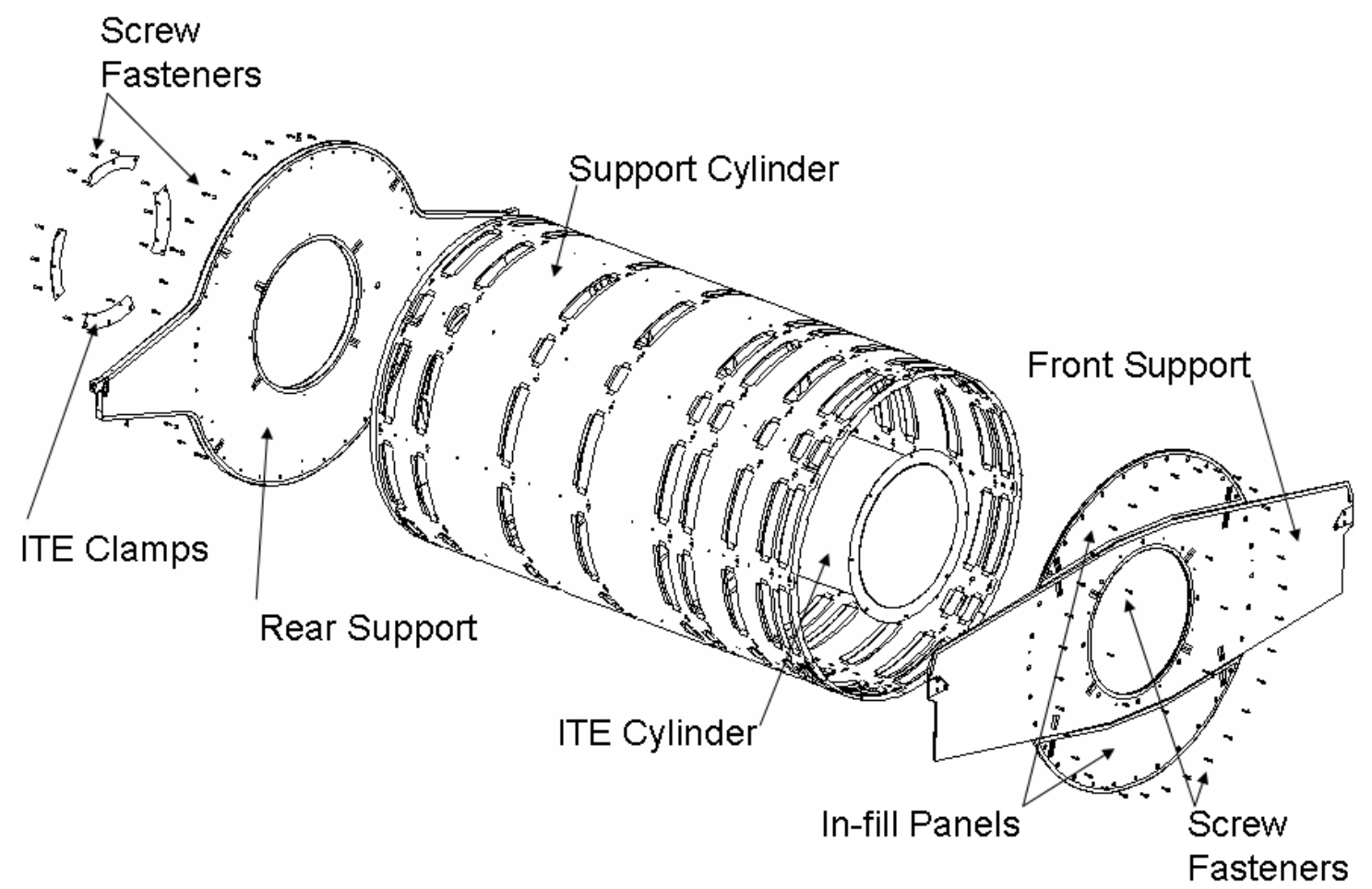

Figure 22. End-cap support structures, showing the main components.

outs were used for the support cylinder and aluminised polyimide films on the support panels. All these materials, especially the YSH-50A fibres and cyanate-ester, have been used in order to create structures which are stiff and low mass, with close to zero CTE (coefficient of thermal expansion) and low CME (coefficient of moisture expansion).

\subsubsection{Support cylinder}

Each support cylinder (see figure 23 and figure 35) is a CFRP sandwich cylinder, nominally $1943.3 \mathrm{~mm}$ long and $1165 \mathrm{~mm}$ outer diameter. The sandwich consists of two facesheets, $0.225 \mathrm{~mm}$ thick, and a honeycomb core of $9 \mathrm{~mm}$ thickness. The CFRP facesheets are arranged in three layers: for the outermost layer of the cylinder, the direction of the fibres lies along the axis of the cylinder; in the other two layers of each facesheet, the fibres are arranged at $\pm 60^{\circ}$ to the axis. This results in a quasi-isotropic lay-up, as used for the disks. The cylinder wall is perforated with a number of apertures; the larger rectangular apertures are for services to pass in and out from the patch panels (PPF0) on the edge of the disks (or directly from the disks in the case of the FSI optical fibre ribbons). These rectangular apertures are edge-sealed with moulded CFRP 34 "U" shaped closeouts. The ends of the cylinder are also closed-out with similar CFRP "U" sections. There are smaller, round apertures for the disk fixings (the aluminium alloy inserts, visible in figure 23) and throughholes which have been fitted with Ultem $\AA$ (polyetherimide) 2-part threaded inserts. The ends of

\footnotetext{
${ }^{34} \mathrm{~T} 300$ graphite fibres in an RS-3 cyanate-ester resin matrix, manufactured by YLA Inc..
} 


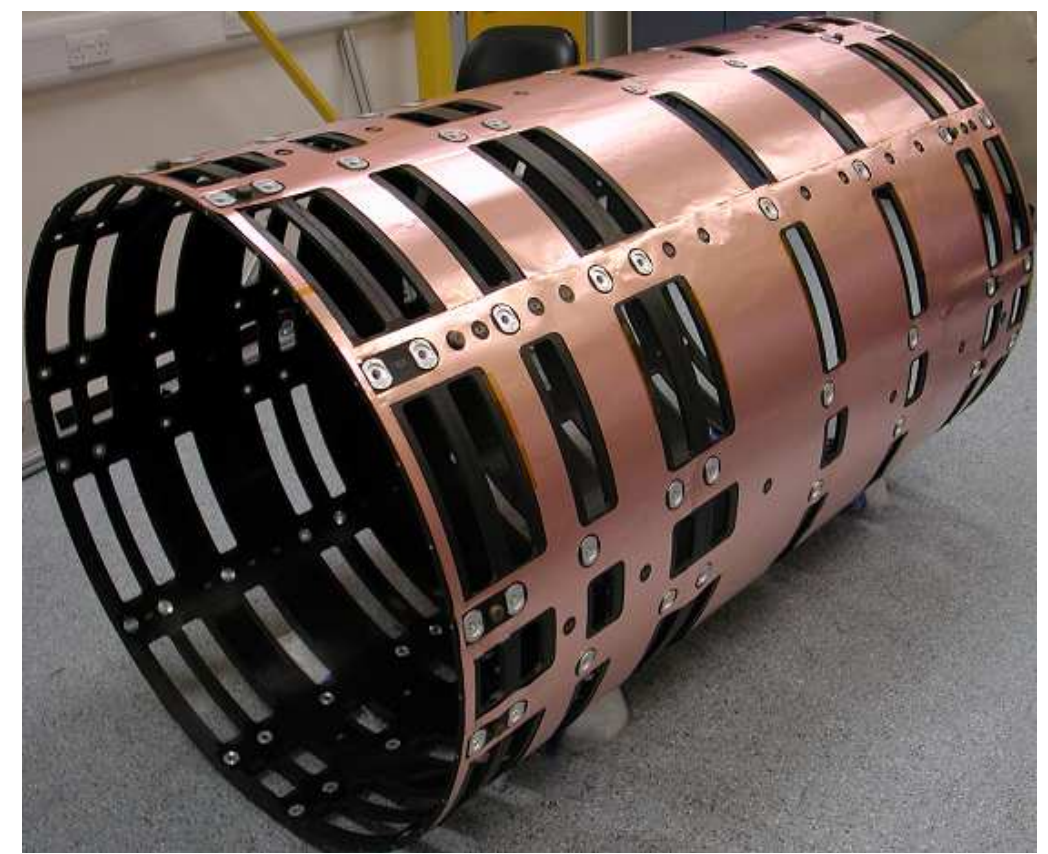

Figure 23. Support cylinder fitted with Cu-polyimide ground sheet.

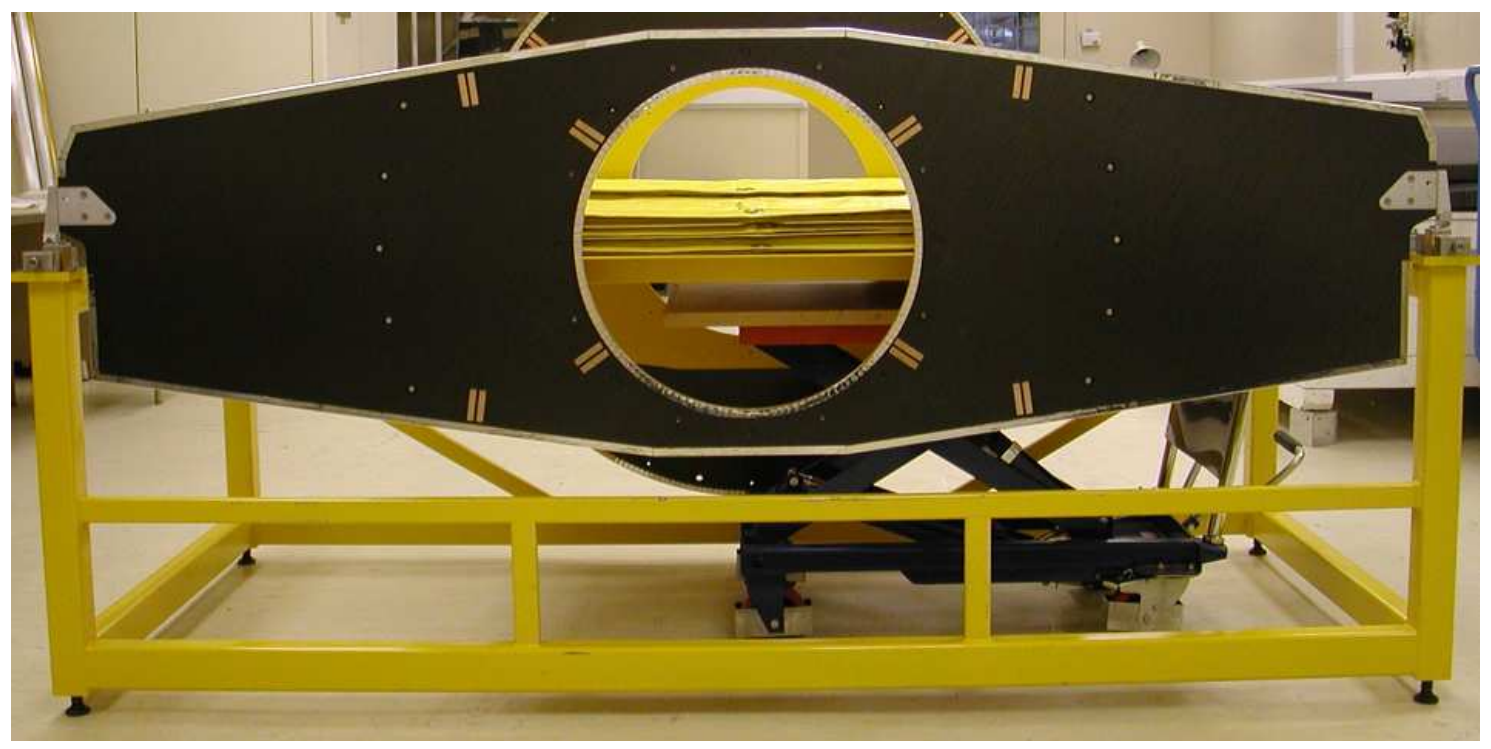

Figure 24. Front support during load testing.

the cylinders have embedded aluminium alloy inserts to support the screw fastenings which attach the front and rear supports.

\subsubsection{Front and rear supports}

The front and rear supports are both flat CFRP sandwich panels consisting of a central circular 


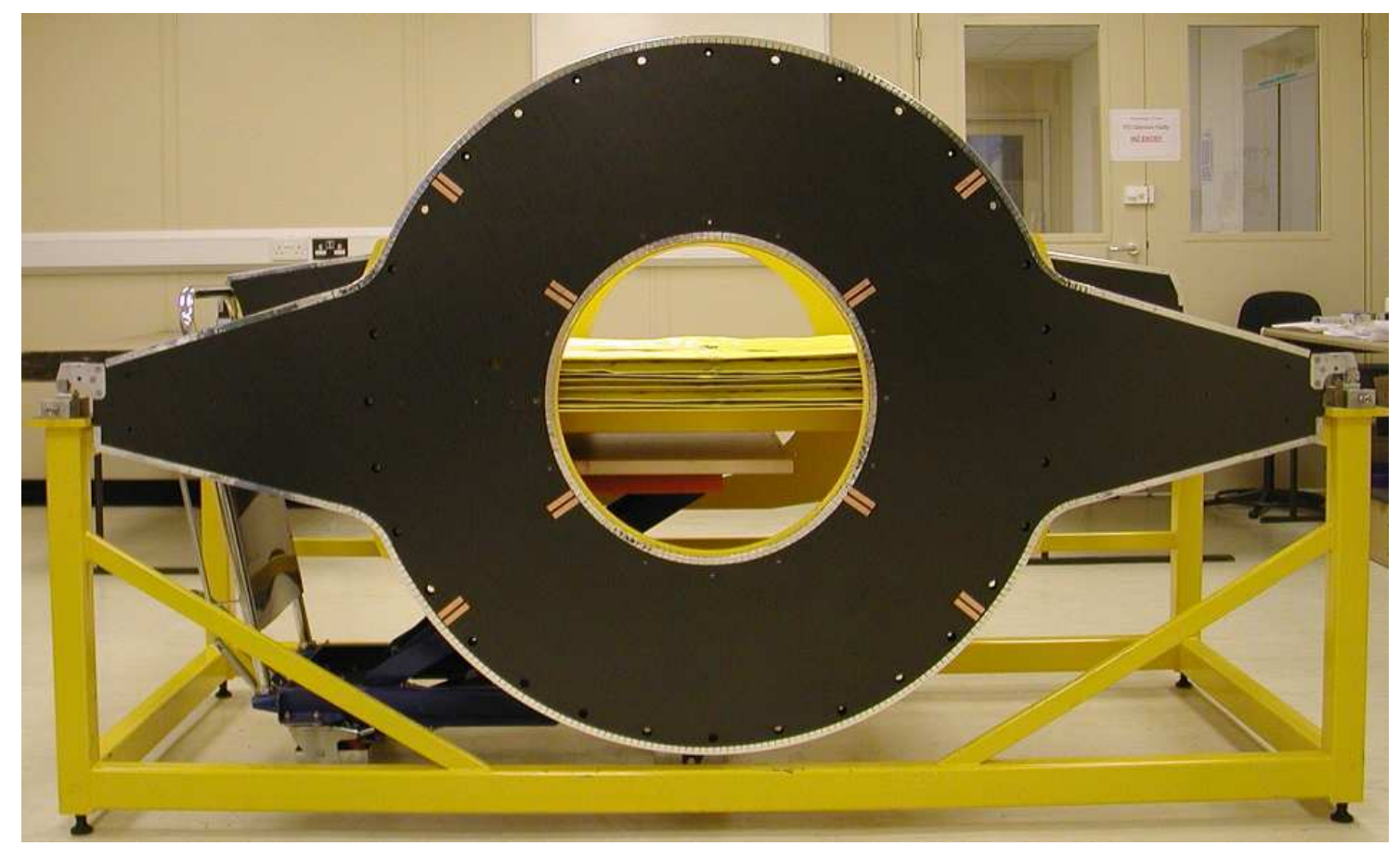

Figure 25. Rear support during load testing.

section of $1213 \mathrm{~mm}$ diameter and two support arms extending from this central section to a width of $2190 \mathrm{~mm}$ at the tips of the aluminium alloy mounts for the support mechanisms (see below). The support panels have integral inserts to strengthen locally the sandwich panel where it is bolted to the support cylinder. Another feature of both panels is the central aperture: this, along with the inner diameter of the ITE, creates space for insertion of the pixel detector and its services at the centre of the inner detector. The edges and apertures of the panels are sealed with aluminised polyimide tape. ${ }^{35}$ The front support is split into three separate sections: a horizontal central part with the arms and two in-fill panels (top and bottom) which complete the central circular section. The split design is essential for integration, where a mix of temporary supports and final parts are interchanged to transfer the SCT end-cap load from the tooling to the TRT end-cap rails, see section 9.2. The front support sandwich consists of two $0.45 \mathrm{~mm}$ thick facesheets with six plies in the quasi-isotropic lay-up of $0^{\circ}$ and $\pm 60^{\circ}$, as with the support cylinder and disks. The front support has a core of $8 \mathrm{~mm}$ thickness making the overall panel thickness nominally $9 \mathrm{~mm}$. The rear support needs to support approximately two-thirds of the end-cap weight and consequently is thicker: the sandwich consists of two $0.9 \mathrm{~mm}$ thick facesheets (twelve plies, $0^{\circ}$ and $\pm 60^{\circ}$ ) and a core of $25 \mathrm{~mm}$ thickness, giving a nominal panel thickness of $27 \mathrm{~mm}$.

The support structure is sensitive to vibrational excitation which may occur within the experiment. This is especially significant in the axial (z) direction of the experiment where volumetric constraints within the inner detector only allowed for support using thin panels. The extra thickness of the rear support core significantly stiffens the panel, raising the first fundamental frequency of

\footnotetext{
${ }^{35}$ Supplied by Sheldahl.
} 


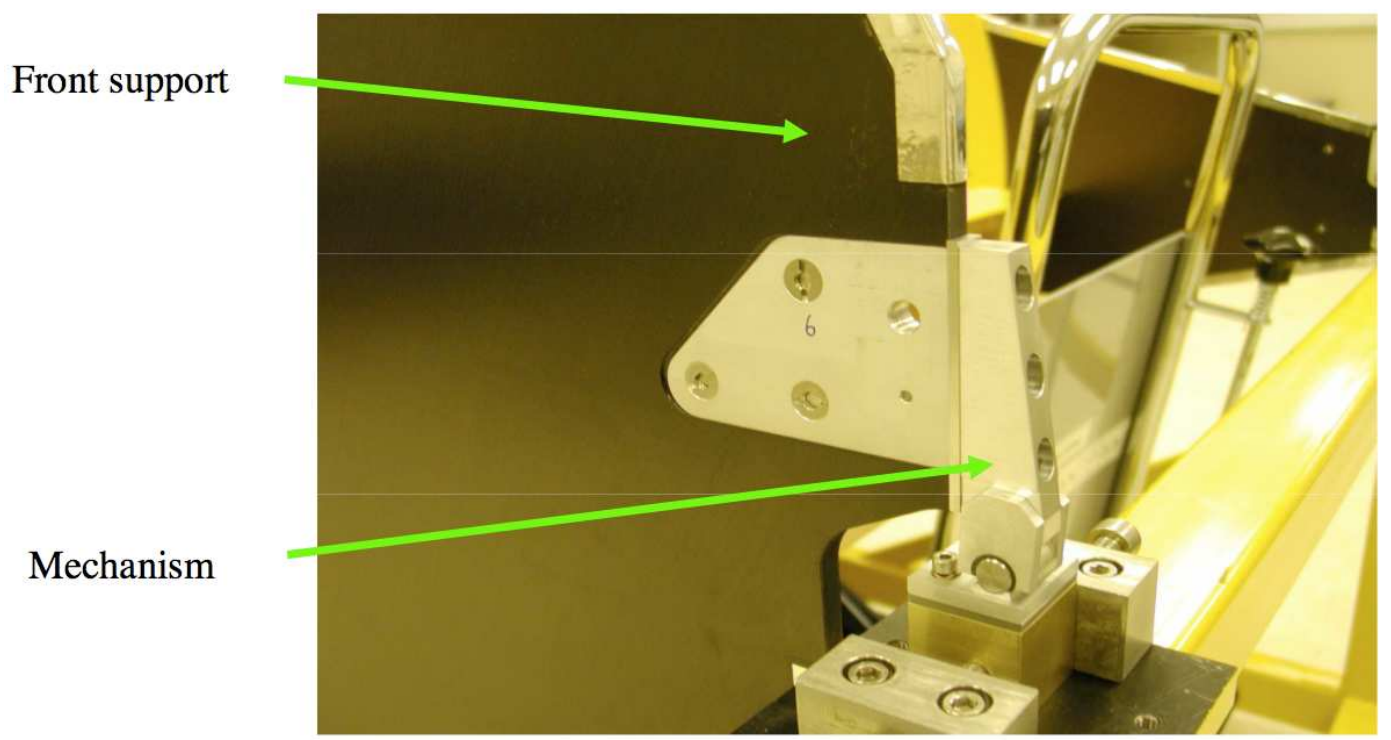

Figure 26. One support mechanism attached to a front support panel during load testing.

the support system. It was found during analysis of the system, as well as during testing, that for lower modes, the amplitude of oscillations is insignificant in relation to the stability required.

\subsubsection{Support mechanisms}

The support mechanisms (see figure 26) are mounted on the end of the protruding arms of the front and rear support panels. The mechanism assemblies (mainly aluminium alloy) incorporate plain bearings which allow different degrees of freedom of the structure during contraction or expansion - these dimensional changes are caused by temperature or moisture changes (e.g. acclimatisation to operational temperature in the dry gas environment). The mechanisms support the full mass of the end-cap including all the internal services and thermal enclosure; they even support a proportion of the external services and services management (cable trays) up to PPF1. The end-cap mass is distributed at four points in the horizontal plane. The support constraints for each end-cap are shown in figure 27.

\subsubsection{ITE cylinder}

The ITE cylinder is a single CFRP laminate; ${ }^{36}$ there are five plies in the laminate giving a total thickness of $0.58 \mathrm{~mm}$. The dimensions of the bare cylinder are nominally $2009 \mathrm{~mm}$ long, $507.6 \mathrm{~mm}$ inside diameter. As part of the thermal enclosure, its outer surface is covered with a layer thermal insulation, the gas purge inlet system and finally a ground sheet - see section 8.4.3. The front end of the cylinder is fitted with a $1.5 \mathrm{~mm}$ thick CFRP flange (13 plies of the T300 plain weave), while the other end is attached to the rear support by the ITE brackets.

\footnotetext{
${ }^{36} \mathrm{~T} 300$ graphite fibres in an RS-3 cyanate-ester resin matrix, manufactured by YLA Inc..
} 


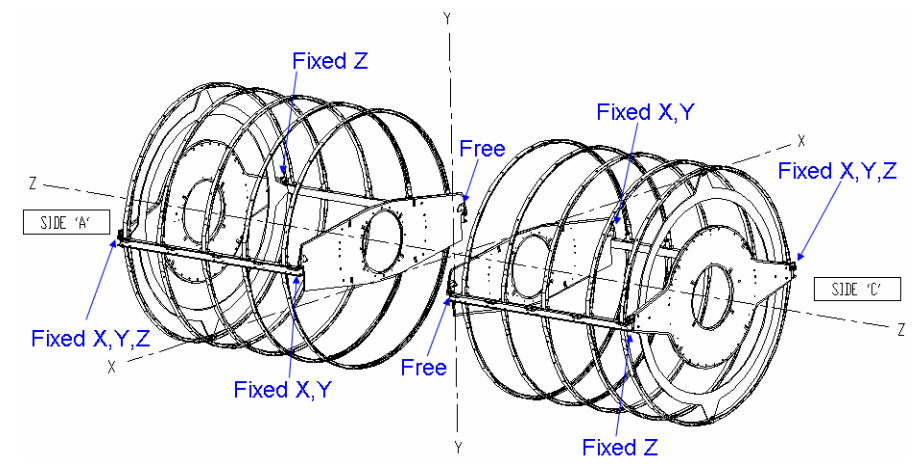

Figure 27. Support constraints imposed by the mechanisms on the two end-caps.

\subsubsection{FEA}

A detailed model of the end-cap [40] using ANSYS $^{37}$ was constructed to study the support structure. This consisted of 37000 elements and included the disks supported by stiff (in lateral direction) springs and allowed for the services on the cylinder. Simulations were run at room temperature and at operating temperature - the differences were small due to the high-performance low-CTE materials used. The effect of irradiation was not analysed, but proven radiation hard materials were used for the construction, and a large safety-factor of two was used to allow for any degradation in the structural properties of the material.

The overall CTE was found to be $1.4 \times 10^{-6} /{ }^{\circ} \mathrm{C}$, similar to that estimated for the disks; while the strain (CME) was estimated to be $1.0 \times 10^{-4}$ for a $50 \%$ increase in relative humidity at room temperature. It is expected that when the SCT is cooled and dried to the operating conditions, the shrinkage of the support cylinder will be around $300 \mu \mathrm{m}$. The maximum facesheet stresses due to cooling to the operating temperature and the effects of gravity were estimated to be $35 \mathrm{MPa}$ at the "wing-tips" of the front support, compared to a maximum allowed stress of $70 \mathrm{MPa}$. This does not allow for the additional strengthening incorporated in this region. The maximum deflections under these conditions correspond to a sag of the cylinder and disks around the position of disks 4 and 5 of about $0.5 \mathrm{~mm}$, as shown in figure 28. When CME effects are considered, due to the drying

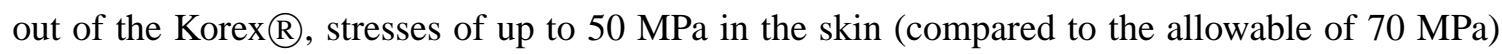
and $0.26 \mathrm{MPa}$ in the core (compared to the allowable of $0.36 \mathrm{MPa}$ ) may be encountered at the end of the support cylinder. The allowables have a further factor of safety of two before failure. Temperature variations of $2^{\circ} \mathrm{C}$ are expected to result in movements of the disks of no more than $8 \mu \mathrm{m}$ transversely and $15 \mu \mathrm{m}$ longitudinally — well within the tolerances. The fundamental mode is an axial movement at $6 \mathrm{~Hz}$, while the second mode is at $24 \mathrm{~Hz}$ and corresponds to a transverse motion of the end of the support cylinder at the front.

The displacements of the end-cap when subjected to vibrations were studied [41]. The power spectral density (PSD) measured at the LEP accelerator (CERN) [42] has a maximum value of $1 \times 10^{-11} \mathrm{~g}^{2} / \mathrm{Hz}$ at $5 \mathrm{~Hz}$. Using the complete PSD yields displacements of the end-cap which are less than 1 (4) $\mu \mathrm{m}$ perpendicular (parallel) to the axis. Even taking a much harder PSD measured at

\footnotetext{
${ }^{37}$ A trademark of SASIP Inc., supplied by ANSYS Inc..
} 


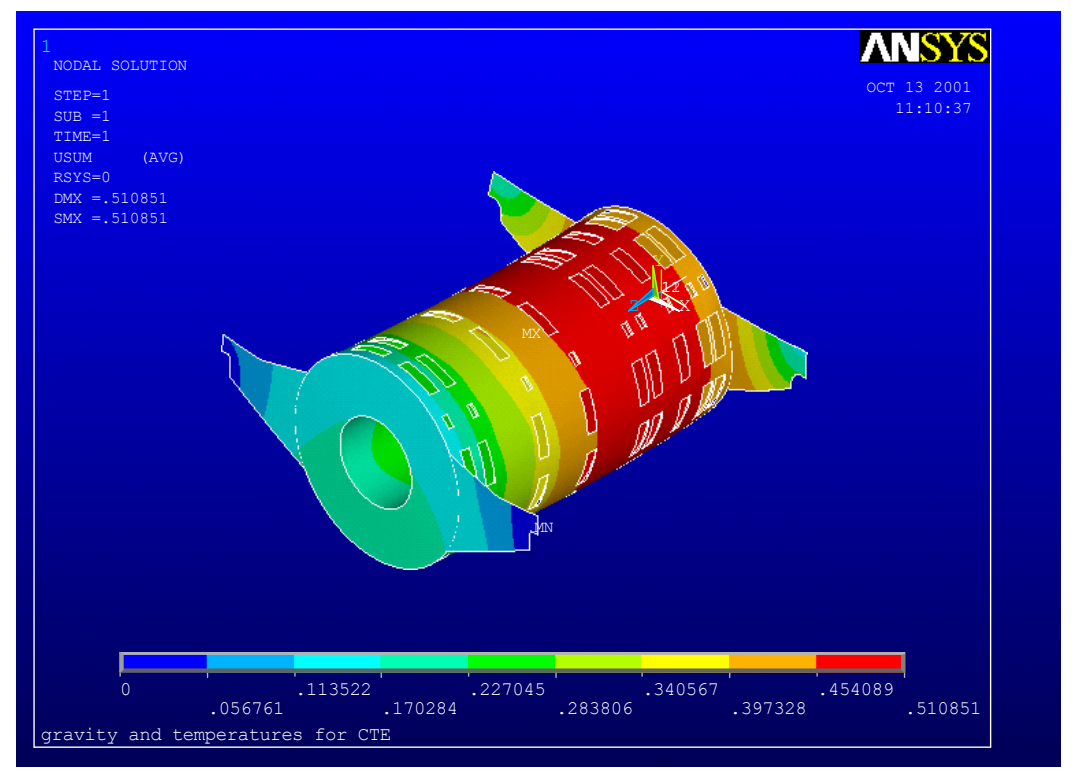

Figure 28. FEA showing the effect of gravity and CTE.

the Daresbury SRS where the maximum value is $1 \times 10^{-8} \mathrm{~g}^{2} / \mathrm{Hz}$, the maximum displacements are only 3 (40) $\mu \mathrm{m}$ perpendicular (parallel) to the axis. These would not affect the statistical precision of the SCT.

\subsection{Manufacture and testing}

The support structures were manufactured by Programmed Composites Inc. (PCI). In a qualification step, a one-third length prototype of the support cylinder, including apertures, was constructed along with a number of test panels. The one-third length cylinder underwent metrology to qualify the tooling and manufacturing process. The test panels were subjected to ultrasonic examination (to check for delamination), thermal cycling and CTE/CME tests as well as bend tests and flatwisetensile tests (FWT).

The support cylinder sandwich was co-cured in an autoclave on a cylindrical steel mandrel whose dimensions were chosen to compensate for thermal expansion during the co-curing process at $175^{\circ} \mathrm{C}$. The apertures and insert holes were machined one quadrant at a time using a template which ran along the length of the cylinder. The cylinder was indexed quadrant by quadrant in a step-and-repeat machining process. Finally the closeouts were applied along with the various threaded inserts. The laminate ITE was made in a similar way on a smaller steel mandrel. The front and rear support panel facesheets were pre-cured and assembled with the core and epoxy film adhesive on a surface table. All of the structures had copper pads embedded in their surface during curing to allow electrical connection to the conductive CFRP facesheets for grounding purposes.

The completed structures underwent laser metrology as well as a "coin-tap" test to test the integrity of the sandwich structure, and in particular the adhesion of the facesheets. Furthermore, test samples were cut from the same panels from which the front and rear supports were produced, and these samples were subjected to bend and FWT tests. 
Preliminary tests by PCI led to the understanding that the core needed to be dried to avoid moisture contaminating the film adhesives. The first flat panels produced failed the FWT tests (require $>2.5 \mathrm{MPa}$ ), with bond failure in the film adhesive. This was attributed to moisture egress from the Ultem $\AA$ inserts which were subsequently replaced with graphite. A second failure was attributed to the cyanate-ester based RS4 film adhesive because of its sensitivity to moisture. For the front and rear supports, this was subsequently replaced with FM73U with successful results.

While at the manufacturer's premises, the complete sets of structures for each end-cap were tested to ensure that they fitted together and could support a load corresponding to the estimated weight of all of the disks and associated services plus a margin of safety. The load was applied by draping flexible lead blankets over the cylinder. The lead blankets ensured the load remained evenly distributed over the cylinder as it deformed and that it was not subjected to point loading. A proof load of more than 1.5 times working-load was applied. During the loading a laser tracker was used to measure deflections. Maximum deflections of $0.74 \mathrm{~mm}$ and $0.87 \mathrm{~mm}$ for the two structures were measured, in reasonable agreement with the FEA prediction of $0.63 \mathrm{~mm}$.

The most important features on the support cylinder are the holes for the fixings (see figure 35) which position the disks. These were required to be located to $0.25 \mathrm{~mm}$. It proved difficult to measure these positions on a large structure which would only be rigid after assembly of the support panels. Ultimately this measurement was achieved by surveying the cylinder on a rotary table in a naturally relaxed state, namely standing vertically on its end. It was found that the holes were displaced by up to $2 \mathrm{~mm}$. This was attributed to the difficulty of machining apertures and fixing holes in quadrants using the step-and-repeat process - the main cause of the problems being the location of the cylinder's axis. Rectification was achieved by enlarging the holes and then precisely placing the fixings using the rotary table, a theodolite and an accurate tooling bar.

As a final step, a Cu-polyimide (18 $\mu \mathrm{m}$ copper, $25 \mu \mathrm{m}$ polyimide $)^{38}$ ground sheet was placed over the cylinder to provide a solid electrical ground (see figure 23). Care was taken to ensure that this had some slack so that when the SCT is cooled to its operating temperature, the sheet does not stress the support cylinder or rip.

\section{Cylinder services}

Figure 29 shows the service apertures for disks 1, 2, 3 and 4 (from left to right). The Cu-polyimide ground sheet can be seen on the CFRP support cylinder. Above and below the apertures are the disk fixing springs of which there are 12 per disk. Their design allows the support cylinder diameter to change by a few microns whilst keeping the disks coaxial. Outside the disk fixings are the nitrogen exhaust-circuit pipes (transparent PEEK tubes with an aluminium alloy manifold which can be seen on the left of the picture), and outside those are the low-mass power tapes (LMT's) with their associated cooling pipes and heat transfer foils. Running along the centre between the disk fixings are the small-diameter inlet capillaries for the evaporative cooling and the associated large diameter exhaust pipes. The rails for the outer thermal enclosure pass over these capillaries and pipes.

\footnotetext{
${ }^{38} \mathrm{Cu}$-polyimide foil (18 $\mu \mathrm{m}$ copper, $25 \mu \mathrm{m}$ polyimide), manufactured by GTS Flexible Materials Ltd..
} 


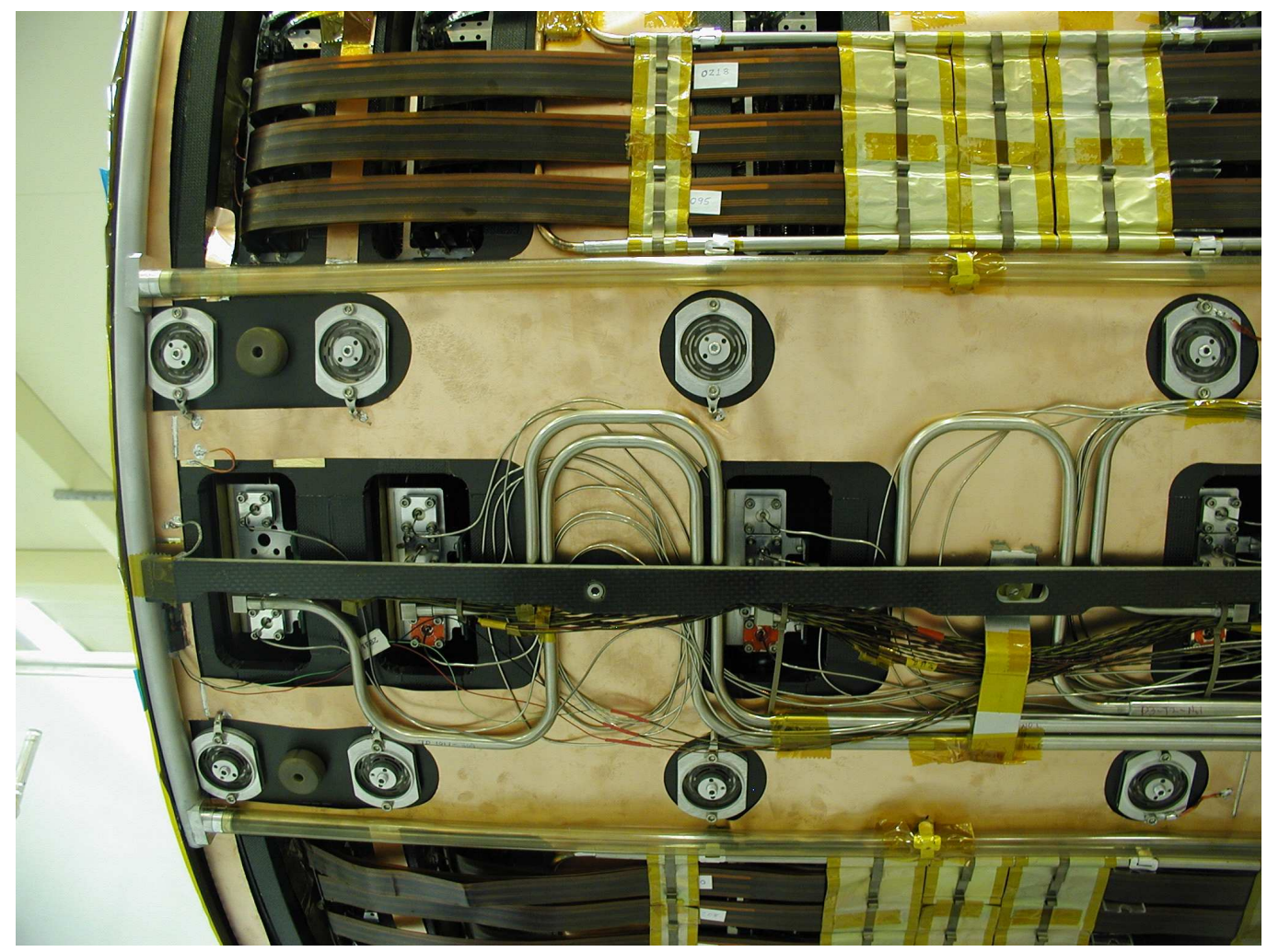

Figure 29. Services on the end-cap support cylinder (see text for details).

\subsection{Evaporative cooling interconnects}

The evaporative cooling interconnects run from the PPF0's located on the disks to the services thermal feedthrough (STFT) at the end of the support cylinder. The inlets are $\mathrm{Cu}-\mathrm{Ni}$ capillaries of inner diameter $0.68 \mathrm{~mm}$ for inner and middle module circuits and $0.76 \mathrm{~mm}$ for outer module circuits; both types have $0.2 \mathrm{~mm}$ wall-thickness. The capillary lengths were tuned to ensure that the pressure drops (and corresponding mass flows) were the same for all capillaries of a given nominal diameter. To ensure the stable operation of the heat exchangers, the larger diameter capillaries were also used for the middle module circuits of disk 1. Excess capillary lengths were accommodated by coiling the capillaries on the surface of the support cylinder, as can be seen in figure 29 .

The exhaust circuits are made up of $\mathrm{Cu}-\mathrm{Ni}$ tubes of two different inner diameters: $6 \mathrm{~mm}$ (with a $200 \mu \mathrm{m}$ wall thickness) and $8.1 \mathrm{~mm}$ (with a $150 \mu \mathrm{m}$ wall thickness). The circuits include a large "wiggle" (see figure 29) to minimize the forces transferred onto the disks via the PPF0 patch panel when the tubes contract during operation. The smaller diameter pipes are used for the wiggles; the thicker wall is a manufacturing requirement to cope with the production of the tight bends without collapse. The larger diameter pipes were used for the straight runs: the larger diameter maximises fluid throughput, minimises pressure drops and reduces material by the use of the thinned pipe walls. 


\subsection{Low-mass tapes}

The low-mass tapes (LMTs) provide the power and control signals for the modules. They connect the external cables at the PPF1 patch panels at the end of the inner detector to the PPF0 patch panels at the outer radius of the disks. The LMTs were fabricated from polyimide with aluminium tracks in order to minimize the radiation length. However, after mounting the LMTs on the cylinder, there were many cases of cracks forming in the nickel-plated regions at the end of the tapes [43]. Therefore it was decided to change to copper LMTs. These were made from an adhesiveless polyimide material ${ }^{39}$ with a $36 \mu \mathrm{m}$ copper layer - these turned out to be very robust. The tracks used for the high current LV lines were $4.5 \mathrm{~mm}$ wide; $0.5 \mathrm{~mm}$ wide tracks were used for the $\mathrm{HV}$ and all the low current lines. The voltage drop for the longest LMT with the maximum $1.3 \mathrm{~A}$ current will be $83 \mathrm{mV}$.

From each azimuthal PPF0 position, up to three tapes are connected to a disk; these build up as they are joined by tapes from successive disks going along the support cylinder, forming a "stack", with up to 27 tapes in a each stack .

Simulations associated with the thermal enclosure (see section 8.4.6) indicated that without cooling, the centre of the stack of LMT's could rise to $50^{\circ} \mathrm{C}$. While this is not problematic for the tapes themselves, since they were bonded together at $90^{\circ} \mathrm{C}$, it would represent a significant heat-load within the SCT. To avoid this, a dedicated cooling system was devised.

Each set of three LMT stacks running the length of the cylinder is cooled by dedicated cooling pipes which are fed from the module evaporative cooling circuit. The heat transfer from the LMTs to the cooling pipes is aided by a set of $150 \mu \mathrm{m}$ foils clipped on along most of the pipe length. The pipes are fed from the cooling circuits of disks 7, 8 and 9 where the missing rings of modules on each disk provide the additional capacity for cooling the LMTs. Each set of three LMT stacks has a pipe on either side: one side is associated with a pipe from the disk 9 cooling, while the other side is associated with disk 7 or 8 . In this way, a failure of the cooling associated with one of the disks 7, 8 or 9 will not jeopardise the operation of all the modules in an azimuthal slice along the length of the end-cap. The $150 \mu \mathrm{m}$ foils which wrap around the tapes are held tightly to the pipes by means of $\mathrm{Cu}-\mathrm{Be}$ spring clips which also serve to compress the tape stack (otherwise air-gaps between the tapes would increase the thermal impedance across the stack and raise the internal temperature). The edges of the foils are covered with a self-adhesive polyimide tape to avoid sharp edges which might rub on the LMTs (because of thermal motion) causing damage over time. There are a number of foils placed longitudinally along each run of tapes, each foil is around $5 \mathrm{~cm}$ wide with a small gap between to allow any trapped moisture to escape. It is essential that no moisture forms and becomes trapped near the power track on the LMTs, since the high voltage combined with the presence of moisture can lead to a surface breakdown of the electrical insulation and damaging short-circuits could occur to low-voltage lines.

Figure 30 shows measurements across a set of three LMT stacks from a trial representing the thickest part of the stacks at the end of the support cylinder. The sections of LMTs were powered with the maximum power density expected and the cooling was provided by room temperature water. To minimise the effect of the surroundings, the test was undertaken in an insulated box. With cooling on two sides, the temperatures across the three stacks were fairly uniform and only

\footnotetext{
${ }^{39}$ Espanex $囚$, manufactured by Nipon Steel Chemical Co., Ltd..
} 


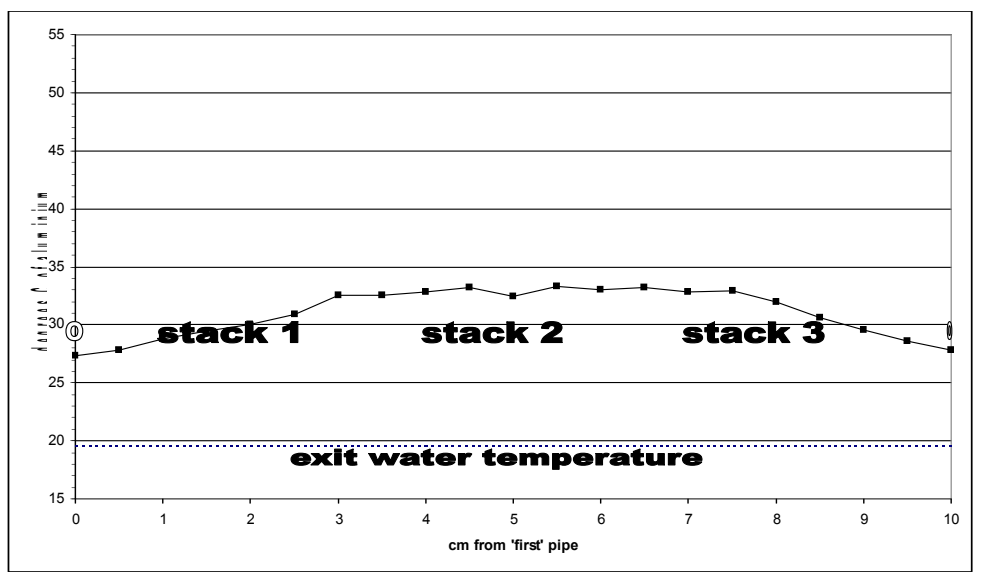

Figure 30. Temperature measurements across a set of three LMT stacks.

about $13^{\circ} \mathrm{C}$ warmer than the coolant. These measurements are indicative rather than definitive, since the heat-transfer coefficient under real operating conditions may prove to be different.

\subsection{Remaining services}

Short ribbon fibres [31] and detector control system (DCS) services also run from the disk PPF0's to PPF1. All the services are held in place by low-mass polycarbonate or aluminium alloy clips.

The nitrogen-purge exhaust pipes are also attached to the support cylinder. There are twelve PEEK tubes with holes drilled along their length which draw in the nitrogen from the SCT end-cap volume. The gas then flows to a circumferential manifold at the front end and then back along the support cylinder and out of the end-cap.

Once all the services were attached, the cooling circuits were tested for blockages and the modules were powered, their electrical and optical circuits read out, checked and repaired as appropriate. The completed assembly is shown in figure 31 .

\section{End-cap assembly}

The end-caps were assembled at The University of Liverpool (UK) and NIKHEF (Amsterdam, The Netherlands).

\subsection{Support of the end-caps}

The end-caps were supported on an assembly frame made from aluminium sections. Two invar rings, the stub supports, were attached to the ends of the support cylinder using the same screw holes to which the final front and rear supports would later be attached. In the absence of the final supports, these rings ensured that the support cylinder remained cylindrical and provided mounting points to the assembly frame. These rings were in several sections, with cut-outs so as not to cover the complete end of the cylinder - this was essential for the integration described in section 9. The support cylinder was mounted onto an assembly frame supported by the stub supports. Using four quasi-kinematic mounting points on the assembly frame, the cylinder was aligned so that its centre 


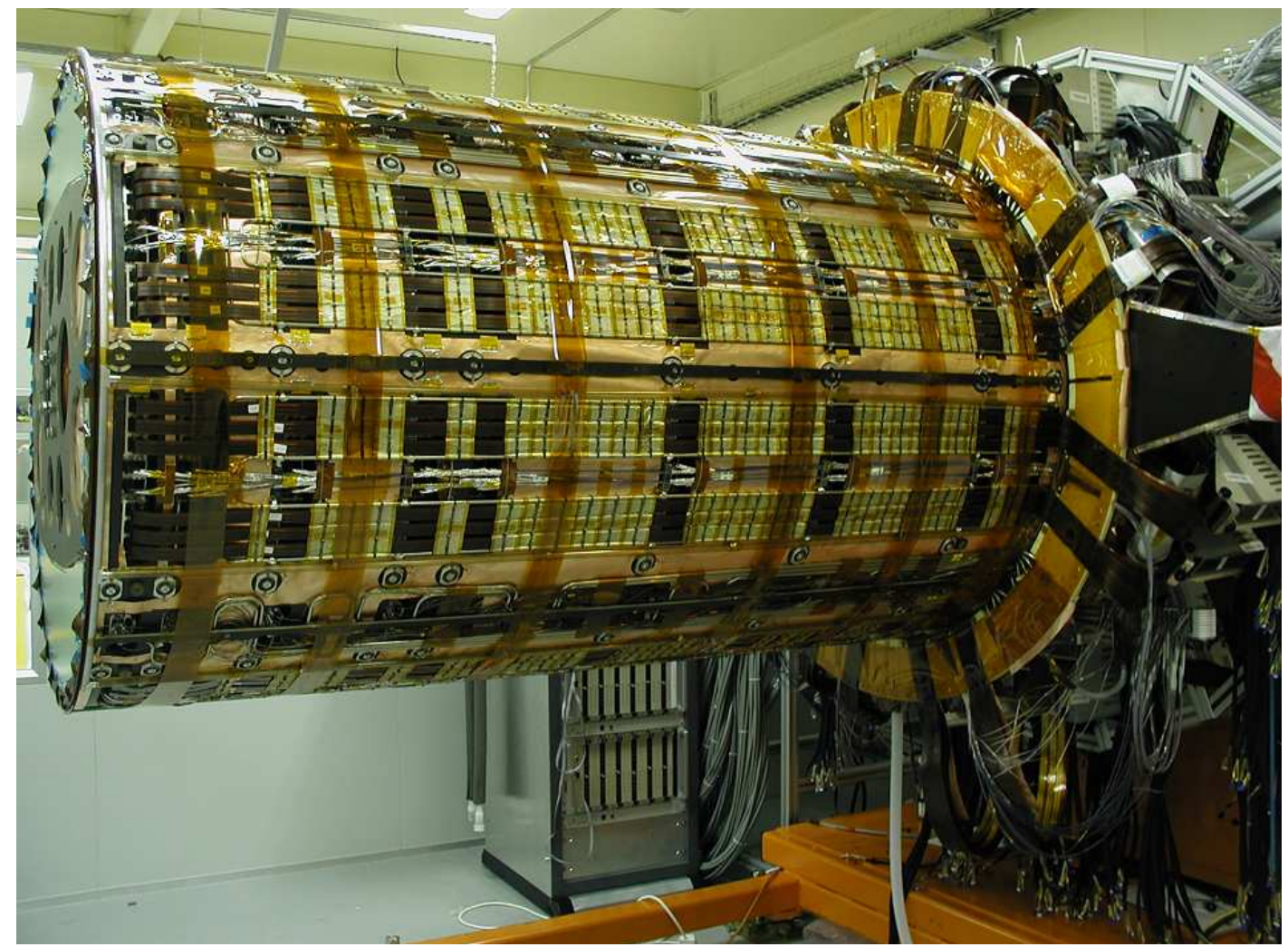

Figure 31. Services on the support cylinder before the outer thermal enclosure was added. (Note this photograph was taken at CERN at the stages described in section 9.1, but it is used here for its clarity.)

coincided with the top of the central beam. A tool to position the disks, the disk grabber, was then assembled onto the central beam and a blank disk (before services had been added) was placed in the tool which was then run along the length of the cylinder to provide the final alignment of the cylinder with respect to the central beam - see figure 32 .

\subsection{Disk insertion}

A sturdy beam was located coaxially with the support cylinder, supported from the rear support frame. The beam supported the disk grabber, a carriage which allowed disks to be moved into position inside the support cylinder by use of a manual belt drive. The disk grabber consisted of three brass fingers which were machined to fit into the groove on the inner surface of the disk. The fingers were mounted on two micrometers which allowed the disk to be moved horizontally and vertically, and an additional mounting was used to rotate the disk about the centre line of the cylinder — see figure 33 .

To insert a disk, each disk, mounted in a support ring in the test box, was brought to the front of the support cylinder. The disk was then removed from the test box and the support ring holding the disk was then connected by locating pins to the stub support. The disk was transferred to the disk grabber and the support ring removed. The disk was then translated by the micrometers until its centre was concentric with the centre of the cylinder. Next it was moved along the cen- 


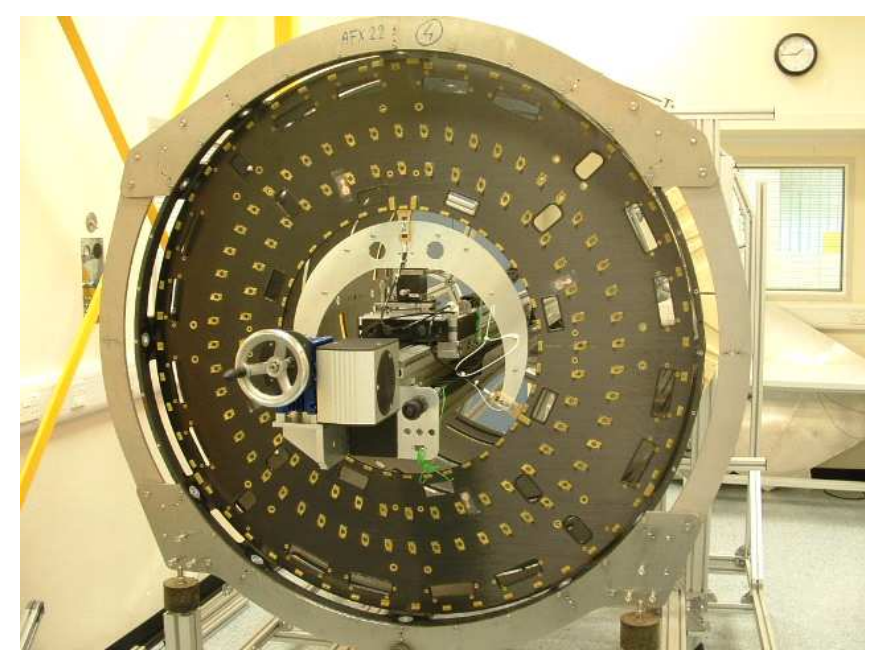

Figure 32. A blank disk mounted in the disk grabber to align the cylinder and beam. The front stub support can be seen at the outer radius - it connects by kinematic mounts to the assembly frame at the lower corners.

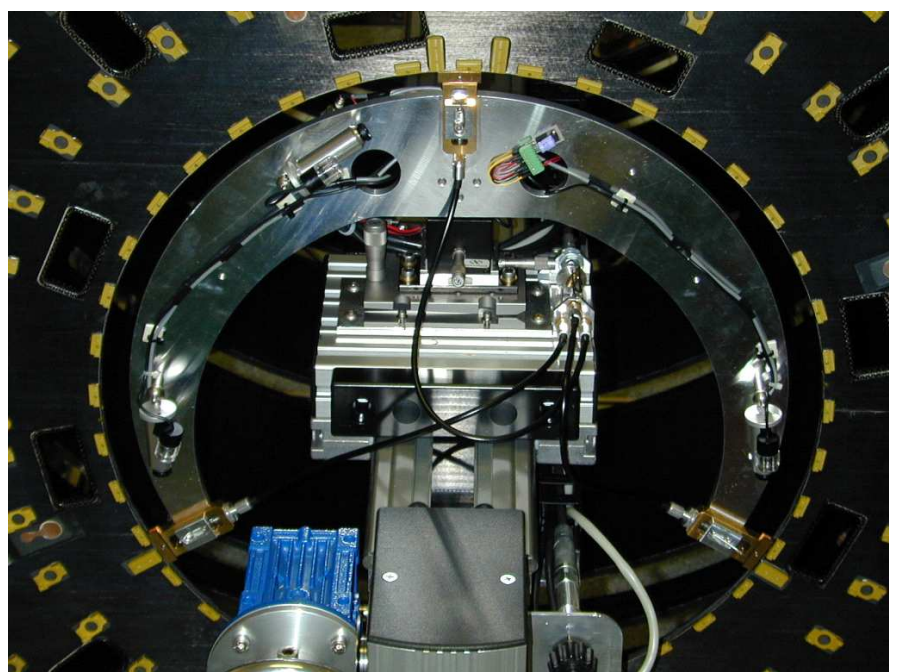

Figure 33. A disk supported by the disk grabber.

tral beam until it was located at its correct aperture by observing fiducial marks on the disk rim through a microscope mounted on a precisely machined bar. Then it was transferred onto a set of kinematic mounts located at 6 o'clock (weight compensator), 12 o'clock (z-movement and weight compensator), 3 o'clock (xyz-movement) and 9 o'clock (yz-movement) - see figure 34 .

The disk was required to be positioned with an accuracy of better than $100 \mu \mathrm{m}$ in $\mathrm{x}$ and $\mathrm{y}$ and $500 \mu \mathrm{m}$ in $\mathrm{z}$ from its nominal position with respect to the cylinder. These are not strong constraints, since the module positions will be corrected by the track-based alignment. Nevertheless, it helps to have a good starting point for these positions, especially in the $x-y$ plane where systematic twists are more difficult to constrain with charged tracks.

The disk was aligned in the $x-y$ plane using two sets of optical targets which were mounted on 


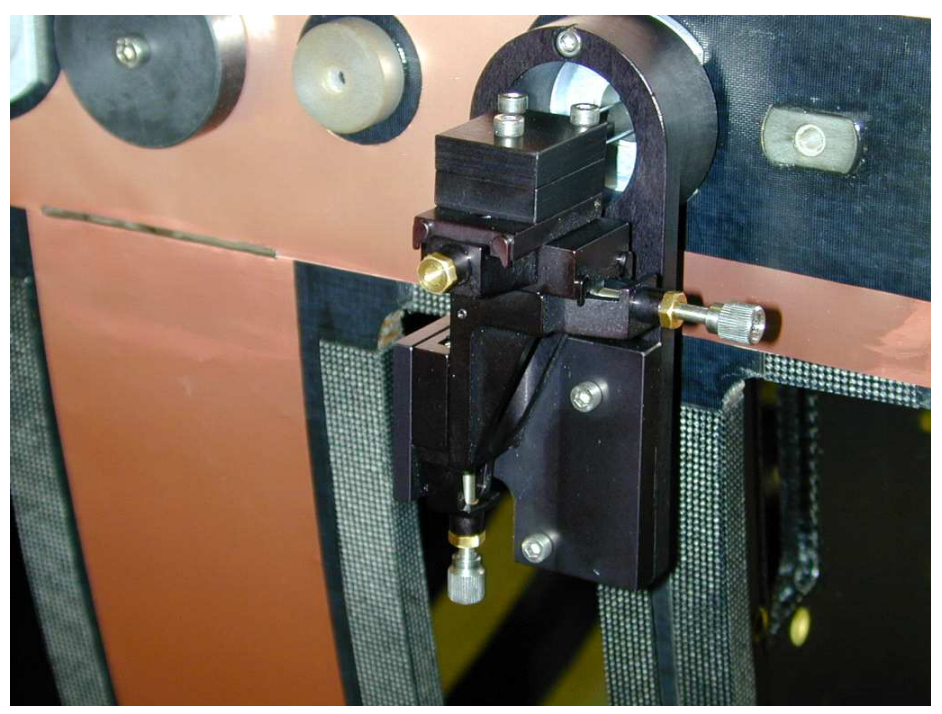

Figure 34. One of the kinematic mounts for capturing a disk.

precision-machined frames attached to the front and rear stub supports. The targets were positioned relative to dowels located in fiducial holes on the end of the cylinder, giving their position relative to the cylinder with a precision of a few tens of microns. A survey telescope was then aligned along the lines defined by the targets. This ensured that the telescope and the targets were aligned along a straight line parallel to the axis of the cylinder and the position of the line in the $x-y$ plane was fixed to an accuracy of $50 \mu \mathrm{m}$ relative to the cylinder. The targets were located in the $\mathrm{x}$-y plane to provide a line of sight through the alignment holes in each disk when the disk was in its correct position. This was achieved by moving the disk on the kinematic mounts until the holes in the disk were located on the line between the telescope and the far target. The accuracy was limited to $25 \mu \mathrm{m}$ by the markings on the target, allowing the disks to be aligned in $\mathrm{x}$ and $\mathrm{y}$ to within $50 \mu \mathrm{m}$ for disk 9 (nearest to the telescope) and $100 \mu \mathrm{m}$ for disk 1 relative to the line defined by the targets.

The alignment in $\mathrm{z}$ was carried out by placing three precision-machined tooling bars (the same bars as used to locate the fixings discussed in section 4.2) on the cylinder, locating them with precisely placed pads at the end of the cylinder. The holes in the bars corresponded to the apertures in the cylinder used for the fixings. These holes held a microscope, allowing it to be located relative to the cylinder with a precision in $\mathrm{z}$ of around $200 \mu \mathrm{m}$. To ensure the accurate placement of a disk, it was adjusted via the kinematic mounts so that its centre line was aligned with the centre of the microscope.

After each disk was positioned inside the cylinder using the kinematic mounts, it was captured by inserting screws into the inserts in the disks - there are 12 of these per disk. The screws are held in position by springs which were designed to ensure that the stress on the disk is minimised. These springs, which are flat and made from copper-beryllium, were attached to the fixings on the cylinder by screws - see figure 35. The screw holes in the springs are over-sized to accommodate small misplacements of the fixings relative to the aligned disk position.

During the alignment of the disks, most of the external services on the support cylinder were 


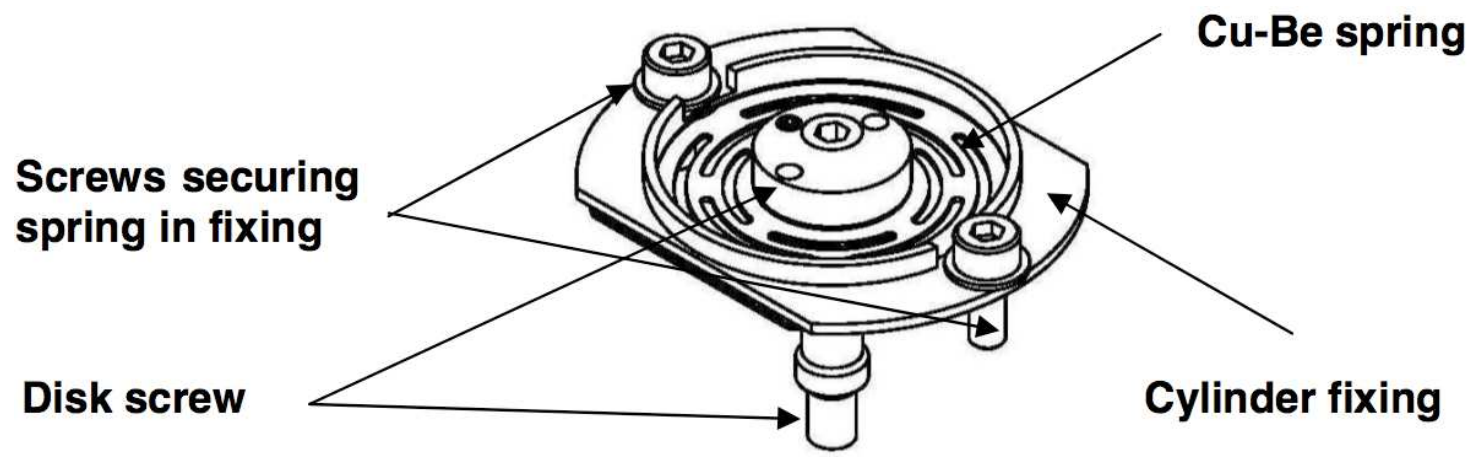

Figure 35. Disk fixing.

absent. Since these are relatively massive, the alignment of the disks would be lost when the services would be added later due to changes in shape of the cylinder. To compensate for the absence of the services, weights were draped along the cylinder to simulate the missing mass.

A final survey of the disks showed that the majority were within around $100 \mu \mathrm{m}$ of the line of sight in $\mathrm{x}-\mathrm{y}$, with the most extreme case being $400 \mu \mathrm{m}$.

\subsection{Services assembly}

The disks were inserted from the front of the end-cap, starting with disk 9 and working back to disk 1. As each disk was added, so the corresponding services (described in section 5) were added to the support cylinder and connected to the PPF0 patch panels on the disk and temporary PPF1's at the rear of the end-cap. The services were held by custom clips (as described in section 5.3) to the cylinder and were potted into the STFT by means of Techsil $\AA$ adhesive. ${ }^{40}$ The silicone-based adhesive was intended to hold the services in place and yet allow them to be removed if necessary. It turned out that when the end-caps arrived at CERN, many of the services needed to be re-laid to ensure that they respected the geometrical envelopes and accurately followed the appropriate paths through the STFT in order to connect to PPF1. It was found that the Techsil@ failed to set sufficiently and so was subsequently replaced with Tempflex $\AA .{ }^{41}$

Both the LMTs and optical fibres were required to undergo $90^{\circ}$ bends at the exit from PPF0 on the disk onto the cylinder and at the STFT at the end of the cylinder, where services exit the endcap onto external cable trays. The LMTs and optical fibres had well defined minimum bend radii to avoid micro-cracking and these had to be respected at both points. For the LMTs, the geometry of the exit at PPF0 led to a natural bend that conformed to the minimum bend-radius but, at the STFT, a jig with correct bend-radius was required to ensure that each LMT in the stack conformed to the minimum bend-radius, otherwise the envelope would have been violated and it would have been difficult to seal the STFT to make it gas-tight. For the fibres, the same considerations were true. In addition, at PPF0, the fibres were wrapped in aluminium foil at the bend (see figure 36) to avoid light leakage at the bend.

\footnotetext{
${ }^{40}$ Techsil 6166 RTV silicone rubber, manufactured by Techsil Ltd..

${ }^{41}$ Tempflex 5145, manufactured by Loctite.
} 


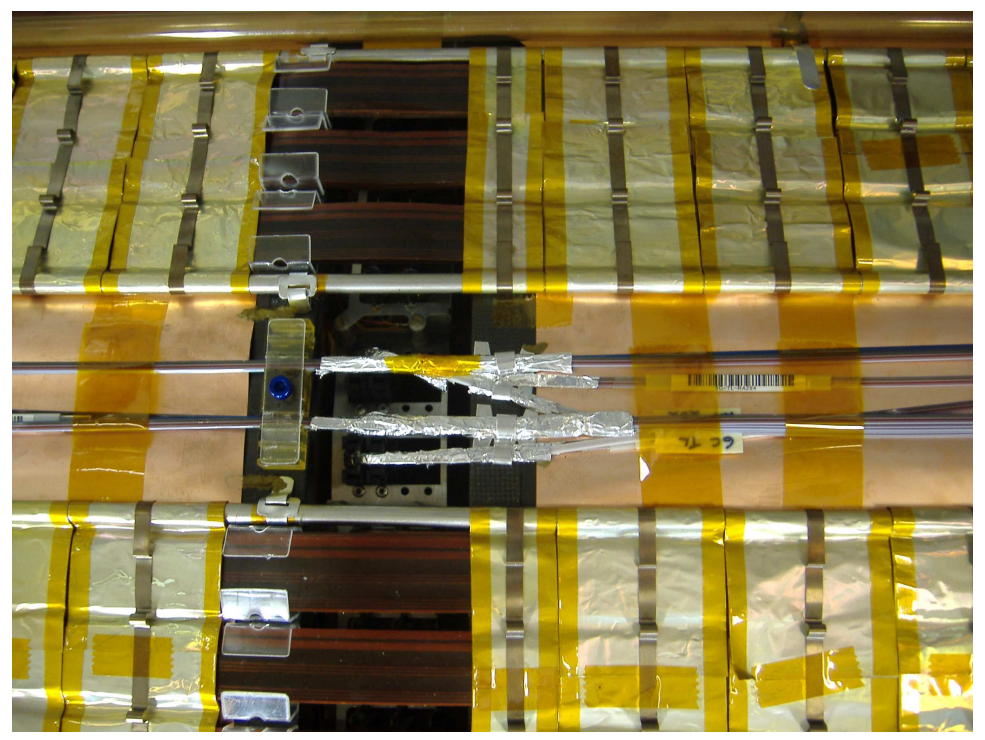

Figure 36. Services at the disk aperture on the support cylinder. Note the optical fibres wrapped in aluminium foil as they exit the cylinder aperture.

Once all the LMTs were laid, they were wrapped in the aluminium foil, as described in section 5.2.

\subsection{Testing during macro-assembly}

As the end-cap cylinders were being populated with fully tested disks, further testing was undertaken to verify the continued excellent module electrical performance. At NIKHEF, the end-cap support cylinder was mounted into a test enclosure. This allowed the performance of individual disks to be checked after each had been installed into the cylinder. At Liverpool, the cylinder was periodically moved into a cold room, allowing disks to be tested in batches rather than one-at-atime.

The testing focussed on checking the integrity of the connections at PPF0 between the disks and the power tapes, fibres and cooling pipes, and then on verifying that the additional components did not cause deterioration in the module electrical performance. In particular, this latter part of the testing was crucial in demonstrating that whole end-cap system design was robust with respect to inter-module pick-up and external interferences. No significant differences were seen compared to the results from the testing of the individual disks. If anything, the noise was slightly better a fact attributed to the use of the LMTs (greater capacitive coupling) and the ground sheet on the support cylinder.

\section{Transportation of the end-caps}

\subsection{Requirements}

The end-caps, when held in the assembly frames, weighed around one tonne and occupied volumes of the order of $3 \mathrm{~m}$ length, $2 \mathrm{~m}$ width and $2 \mathrm{~m}$ height. Because of the delicacy of the silicon 
modules, it was important that the end-caps should be transported very carefully to CERN [44].

It was required that the transportation should take place in an air-sprung, temperaturecontrolled, humidity-controlled lorry. The acceleration experienced by the transport box was required to be less than $3 \mathrm{~g}$ (where $\mathrm{g}$ is the acceleration due to gravity) to avoid damage to the silicon modules and shaking loose connectors. The tilt was required to be less than $10^{\circ}$. The temperature was required to be $20 \pm 3^{\circ} \mathrm{C}$ to avoid thermal stresses and the humidity kept at around $40 \%$ and certainly less than $80 \%$ to avoid condensation forming on the modules.

\subsection{Designs of the transportation boxes}

At The University of Liverpool, a transportation framework was constructed around the assembly framework. To this, sturdy side-panels were attached and the whole assembly was bolted to a large steel "pallet", designed so that the end-cap could be picked up by a fork-lift truck. This enabled the end-cap to be lifted into the waiting lorry in one operation. The completed transport box was $3.7 \mathrm{~m}(\mathrm{l}) \times 2.2 \mathrm{~m}(\mathrm{w}) \times 2.6 \mathrm{~m}(\mathrm{~h})$, and including the pallet weighed 2.8 tonnes. The transportation box is shown in figure 37 .

At NIKHEF, the transport box was formed by the thermally insulating panels which had been used for testing. Again a transportation frame was constructed, and slings were attached under the beams enabling the end-cap to be lifted by a crane onto a platform, from which it was then rolled into the lorry.

For both the end-caps, the assembly frames were supported from the transportation frames by wire-rope isolators. ${ }^{42}$ These look like large springs, but have high damping coefficients in order to reduce vibrations.

Both end-caps were instrumented with accelerometers ${ }^{43}$ to record acceleration in all three directions, as well as temperature and humidity. These were attached directly to the assembly frame as well as the outer box.

\subsection{Transportation}

As a prelude to the actual transportation, dummy loads were constructed to simulate the mass of the end-caps. These even contained a few silicon modules. The loads were instrumented and attached to the transportation frameworks. They were loaded into a lorry using exactly the same procedures foreseen for the end-caps. Tests were undertaken on various road surfaces, at various speeds, on inclines and as a part of emergency braking. The results from the accelerometers indicated that the procedure would be safe.

For the transportation of the end-caps, the wheels of the transportation frames were raised and the boxes were held in position by load-lock bars fixed to the sides of the lorries. For EC-A, additional ballast was added to the lorry to ensure the load carried was at the mid-point of its specified load range. Just before departure, the accelerometers were checked. The transportation proceeded to Geneva along main roads, avoiding the Jura mountains. In the case of EC-C, the transportation was handled from Liverpool to CERN by Danzas-DHL. The end-cap was required to travel by the Channel Tunnel, where the pitch of the ramps to the train is of the order of $6^{\circ}$.

\footnotetext{
${ }^{42}$ Manufactured by Enidine Gmbh..

${ }^{43}$ Shockwatch shocklog micro accelerometers, manufactured by Lamerholm Electronics Ltd..
} 


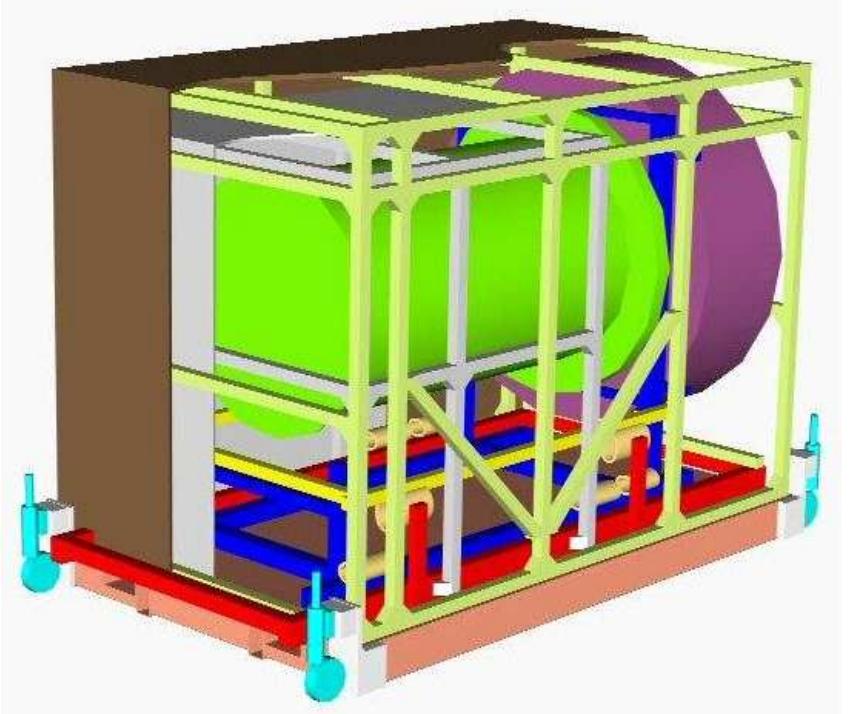

Figure 37. Cutaway showing EC-C (green), the assembly framework (blue), the transportation framework (red) and the pallet (pink).

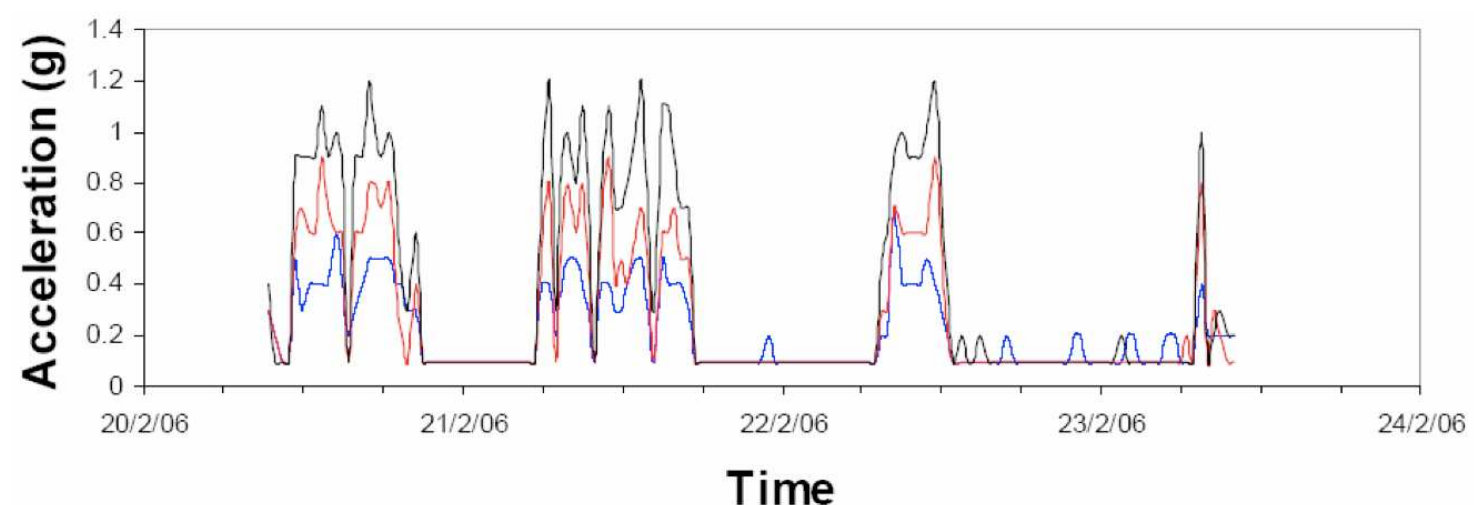

Figure 38. Accelerations in three directions experienced by EC-C during transportation from Liverpool to CERN.

The journeys were carried out at maximum speeds of $80 \mathrm{~km} / \mathrm{hr}$, taking around two days. On arrival at CERN, the accelerometers were checked, the end-caps unloaded using the reversed procedures and then they were subjected to extensive visual inspection. During the journeys, no damage was sustained. Once the lorry was sealed up, the temperature of EC-C remained between $17^{\circ} \mathrm{C}$ and $19^{\circ} \mathrm{C}$, while the humidity did not exceed $60 \% \mathrm{RH}$. The largest acceleration experienced by the inner accelerometers (see figure 38) was $1.2 \mathrm{~g}$, while the outer one experienced $1.8 \mathrm{~g}$.

\section{Thermal enclosures}

\subsection{Requirements}

The end-cap thermal enclosures [45] must be consistent with the requirements set out in section 1.3. The thermal enclosure must: 
- Contain the SCT end-cap environmental gas $\left(\mathrm{dry}_{2}\right)$ and prevent significant flow of different types of gas in and out. The leak rate for each end-cap should be less than $25 \mathrm{l} / \mathrm{hr}$ in order to avoid contaminating the TRT. In this role, the thermal enclosure will also provide a moisture barrier.

This is a very conservative limit on the leak rate, intended to have no effect on the TRT. If the leak rate is exceeded, steps will have to be taken to ensure the TRT is not adversely affected. Provided an overpressure can be maintained, the ingress of moisture can be prevented and the SCT will dry out.

- Provide a thermal barrier such that the heat flow between the SCT and its surroundings is a small fraction of the heat generated within the SCT so that the cooling of the modules is not compromised.

If the heat flow is larger than expected, the module cooling will need to remove more heat; since there is excess capacity, this should not be a problem.

- Ensure that the SCT environmental gas is at $-7 \pm 4^{\circ} \mathrm{C}$ while the external temperature is $22.5 \pm$ $2.5^{\circ} \mathrm{C}$.

If the temperature of the silicon increases, then the radiation damage experienced by the silicon will be greater. Also the leakage current of the SCT increases with temperature, therefore any temperature increase in the silicon temperature will reduce the safety margin against thermal runaway.

- Prevent the formation of ice or condensation on the external surfaces of the SCT in the case of moist gases around the apparatus. This requires that the external surfaces should be maintained above the ambient dew-point of $12^{\circ} \mathrm{C}$. Moist air will be present during access or maintenance or as a fault condition during operation.

The role of the heater pads (see following sections) in maintaining the external temperature is critical. If they fail and moist air is present, then it will be impossible to operate the SCT.

- Provide a Faraday Shield to shield the SCT from outside, and other detectors from the SCT.

- Not affect the stability of the internal supports and hence the modules.

The original concept for the thermal enclosures was based on active cooling, whereby the inner surfaces would be cooled and the outer surfaces heated to maintain a thermal gradient over a short radial distance. This was abandoned for reasons of cost and engineering complexity and it was found that passive cooling through insulation would suffice in the light of the cooling provided by the evaporative cooling exhausts running along the support cylinder; albeit this would need to be complemented by heaters to maintain the external temperature above the dew-point.

\subsection{Design overview}

The end-cap thermal enclosure design [45] consists of

- Outer thermal enclosure (OTE) - a cylinder surrounding the support cylinder and its associated services. 
- Front support - since most of the front of the end-cap faces the SCT barrel, which will be cold, no significant heat flow is expected and hence no significant insulation beyond that provided by the sandwich construction is needed.

- Rear thermal pad - to complement the insulation of the rear support, an insulated pad was constructed.

- Inner thermal enclosure (ITE) - since the inner bore of the SCT end-cap faces the warmer pixel services inside the pixel support tube (PST), the ITE cylinder (see section 4.1.4) was insulated.

To cope with the possible presence of moist air, most of the external surface of the end-cap is covered with heater pads (see section 8.4.4) and heat-spreaders. The volume between the ITE cylinder and the PST will be flushed with dry gas and the PST is instrumented with heater pads.

The layout of one end-cap thermal enclosure assembly is shown in figure 39. The thermal insulation is provided by Airex $\left(\right.$ foam. ${ }^{44}$ This is a closed-cell polyetherimide (PEI) foam which has low thermal conductivity $(0.036 \mathrm{~W} / \mathrm{m} \cdot \mathrm{K})$, low moisture absorption (a few grammes per $\mathrm{m}^{2}$ when in high humidity) and low density $\left(60 \mathrm{~kg} / \mathrm{m}^{3}\right)$. To seal the foam against gas and moisture exchange and provide structural strength, composites with films of either aluminised polyimide ( $<1 \mu \mathrm{m}$ layer of aluminium) or Cu-polyimide (18 $\mu \mathrm{m}$ copper, $25 \mu \mathrm{m}$ polyimide) were applied using Araldite $\AA 2$ 2011. The aluminised polyimide reduces the radiative heat transfer as a result of its low emissivity and the Cu-polyimide provides excellent electrical shielding.

It was proposed to circulate dry nitrogen gas at a rate of up to $1700 \mathrm{l} / \mathrm{hr}$ to ensure that the end-cap can be dried in a few days. To ensure no ingress of carbon dioxide, an overpressure is maintained which will be around 1 mbar, but with a limit of 4 mbar, ensured by a remotely located liquid bubbler overpressure device.

\subsection{Prototyping}

\subsubsection{Glue applications}

The glue used was the two-component Araldite $\mathbb{R}$ 2011. It is important that there is good adhe-

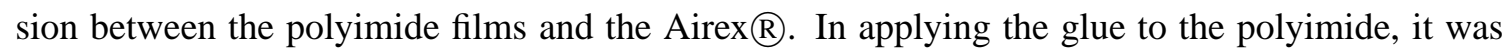
difficult to obtain a uniform layer of sufficient thickness. Instead, better adhesion was obtained by "dabbing" the Airex $₫$ with the glue rather than painting it. Because of the rough surface of the foam resulting from cutting the cells, the Airex had the potential to absorb large quantities of glue. It was found that the peel strength did not vary greatly with glue density, provided a minimum of around $100 \mathrm{~g} / \mathrm{m}^{2}$ was applied - this corresponds to an average thickness of $100 \mu \mathrm{m}$. To reduce the viscosity of the glue, it was found easier to keep the components around $30^{\circ} \mathrm{C}$ and to work with a room temperature of $25^{\circ} \mathrm{C}$. The elevated temperatures increased the cure rate, and this could be reduced by placing the glue in a wide flat tray, since the curing process is exothermic.

\subsubsection{Small cylinders}

Several small $30 \mathrm{~cm}$ diameter prototypes were made - being smaller made the handling of the glue easier but corresponded to a much smaller bend radius and more problems with creasing.

\footnotetext{
${ }^{44}$ Airex $(\mathrm{R} 82.60$ foam, manufactured by Alcan.
} 


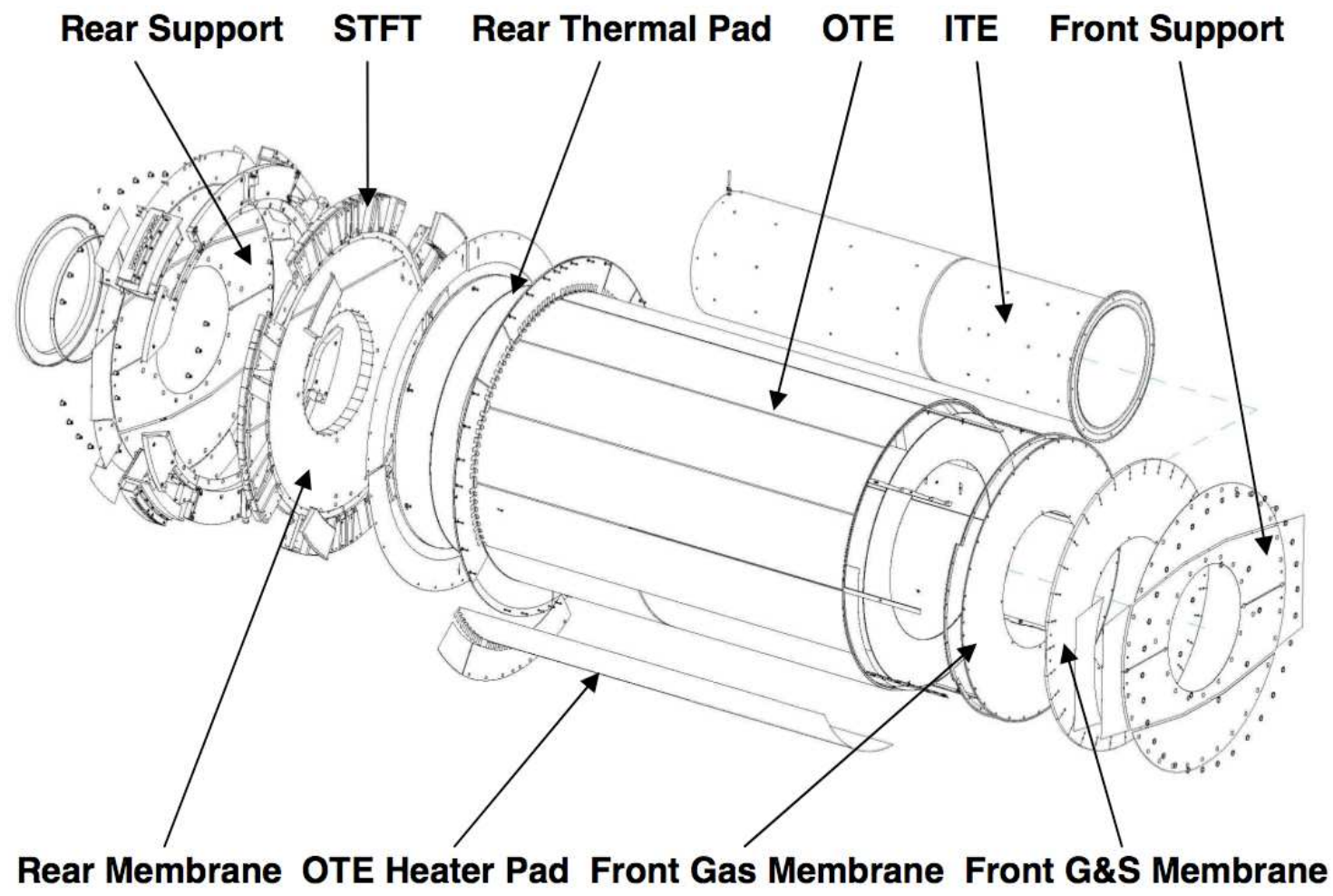

Figure 39. End-cap thermal enclosure assembly, showing the main components.

Several prototypes with the correct diameter but shorter length were also produced, and a $0.5 \mathrm{~m}$ length prototype was assembled in order to evaluate all the assembly steps. The best results for adhesion between the polyimide films and the Airex $₫$ foam were obtained using vacuum-bagging. This worked well for the application of the foam to a layer of polyimide held on a stiff mandrel. However, in applying an outer polyimide layer separately, creases were introduced. Hence it was found best to glue the outer layer of $\mathrm{Cu}$-polyimide to the Airex $\mathbb{R}$ and then when dry, to apply the composite sheet to the inner layer of aluminised polyimide already on the mandrel.

\subsection{Design and manufacture}

\subsubsection{OTE}

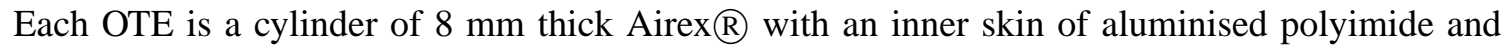
an outer skin of $\mathrm{Cu}$-polyimide. There is an integrated flange in the region of the feedthrough constructed in the same way, albeit with $5 \mathrm{~mm}$ foam, and a $3 \mathrm{~mm}$ wide CFRP ring at the outermost radius where precision holes are located. A second ring made by gluing two $180^{\circ} \mathrm{CFRP}$ pieces was added on the inside of the cylinder.

Both OTE's were constructed in the Instituto de Fisica Corpuscular (IFIC), Valencia. Several sheets of Airex $\mathbb{R}$ were glued edge-ways to form one large sheet, to which $\mathrm{Cu}$-polyimide was glued using a vacuum-bagging technique. Aluminised polyimide was wrapped on a mandrel formed from a $3 \mathrm{~mm}$ steel sheet held by accurate circular ribs. The foam was then coated with glue and wrapped on the mandrel. The foam was $0.6 \%$ longer than the design circumference of the OTE, 


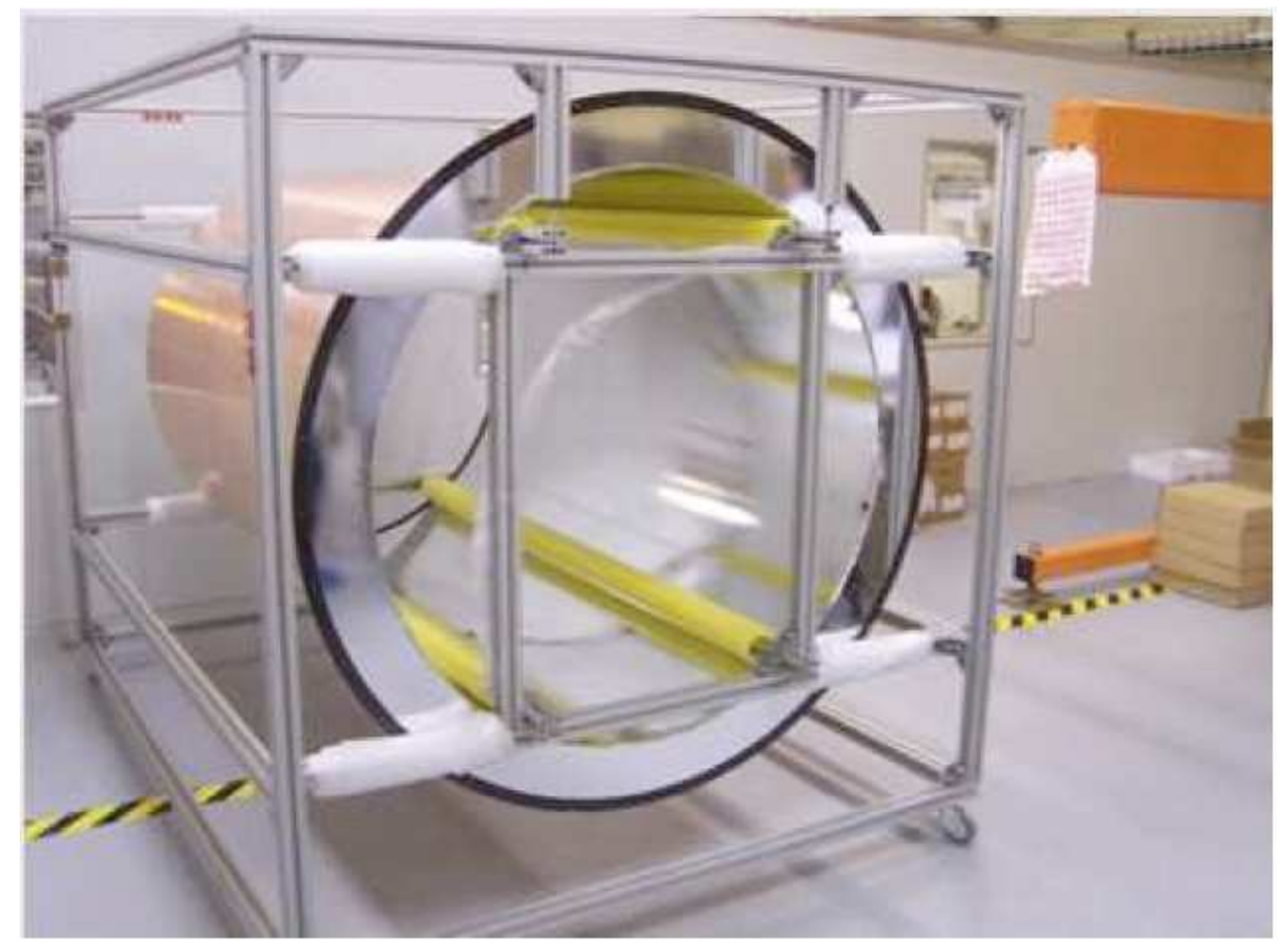

Figure 40. Outer thermal enclosure in its transport frame.

allowing it to be compressed using vacuum-bagging. When the glue dried, this resulted in a very stiff structure. The circular flange was made on a surface table and glued to the cylinder. The inner sheets of aluminised polyimide were connected to each other by $\mathrm{Cu}$-polyimide tabs using a silverloaded glue and jagged chromium strips glued to the cylinder. The outer $\mathrm{Cu}$-polyimide sheets were connected by applying a thin layer of solder covered by a thin strip of copper foil to form a smooth, flat solder-joint of low resistance (discussed further in section 11.1). One completed OTE is shown in figure 40.

Each OTE is held on the support cylinder by eight adjustable composite rails made of CFRP face-skins with Airex $\AA$ cores. These are very stiff but light. All sharp edges were removed, allowing the OTE to be slid easily onto the end-cap.

\subsubsection{Rear thermal pad}

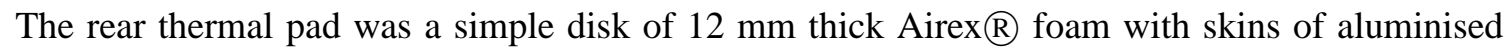
polyimide. Its design is such as to result in a radial gap of about $1 \mathrm{~mm}$ between the inner radius of the support cylinder and the outer radius of the ITE. The pad was cut from a flat sheet with a water jet and the edges were sealed with strips of aluminised polyimide.

\subsubsection{ITE}

Each ITE consists of a CFRP cylinder (see section 4.1.4) covered with $5 \mathrm{~mm}$ thick Airex $\AA$ foam. The foam is covered in $\mathrm{Cu}$-polyimide to complete the Faraday shield surrounding the end-cap. The 


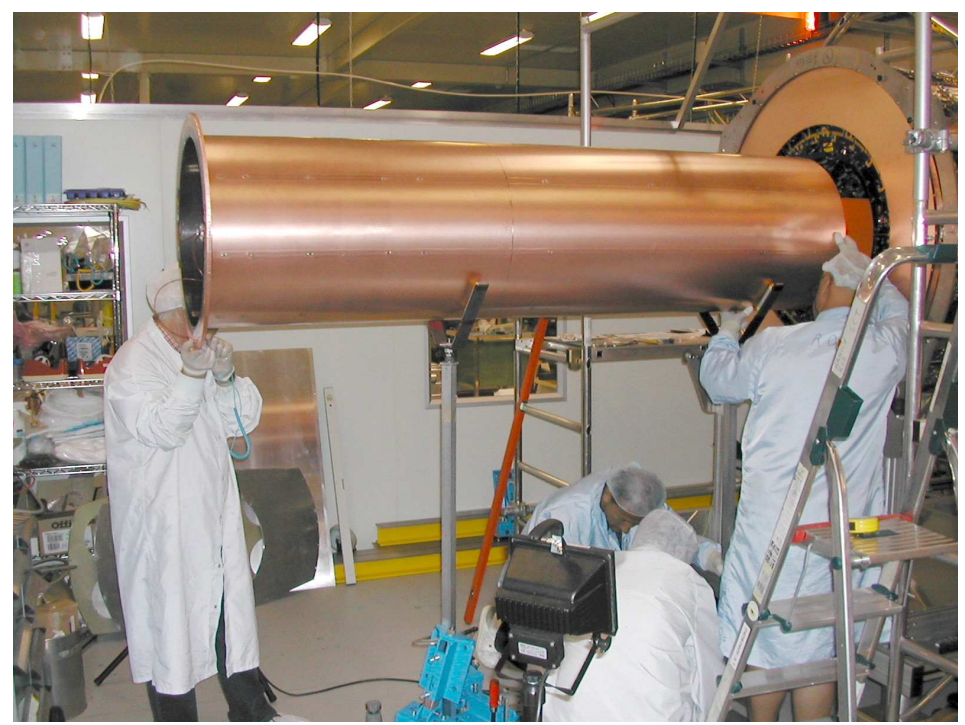

Figure 41. Inner thermal enclosure being inserted into one end-cap.

foam includes eight longitudinal channels which supply the dry nitrogen environmental gas through small apertures. The gas is supplied by a pipe which is external to the end-cap and attached to the ITE at a filter which is intended to prevent the apertures from being blocked.

Both ITE's were completed at CERN. The foam was machined with a router to form the gas channels which were lined with aluminised polyimide. Then the foam was covered with $\mathrm{Cu}$ polyimide. Holes were made in the $\mathrm{Cu}$-polyimide in line with the channels corresponding to the gaps between the disks and small copper disks with precision $300 \mu \mathrm{m}$ apertures were soldered in place. The aperture size was chosen to ensure a sufficient and even flow of dry nitrogen so as to cope with the initial out-gassing of the support structures, avoiding condensation [46]. The complete sheet was then glued onto the CRFP cylinder and compressed with a vacuum-bagging technique. To avoid crushing the thin cylinder, it was supported on the mandrel used for its manufacture. Because the mandrel was slightly smaller in diameter than the cylinder, ${ }^{45}$ when the sheet was applied to the first cylinder, a small bump $1.5 \mathrm{~mm}$ high and $4 \mathrm{~cm}$ long was produced along its length. This was avoided for the second cylinder by wrapping the mandrel with thin sheets of paper. Gas was flowed through the channels before the filter was added to ensure there were no blockages in the apertures. The measured flow was $750 \mathrm{l} / \mathrm{hr}$ - less than the specification of $1700 \mathrm{l} / \mathrm{hr}$. Nevertheless, this is adequate to dry the end-cap and ensure overpressure (see sections 9.3 and 12.1). One completed ITE is shown in figure 41 .

\subsubsection{Heater pads}

To maintain the external temperature of the SCT end-caps, they are covered in thin heater pads. ${ }^{46}$

\footnotetext{
${ }^{45}$ The diameter of the CFRP cylinder was defined by the diameter of the mandrel during the autoclaving process. By design, this corresponded to the nominal diameter, and due to thermal expansion, was larger than the room-temperature value.

${ }^{46}$ Manufactured by Elgoline (Slovenia).
} 


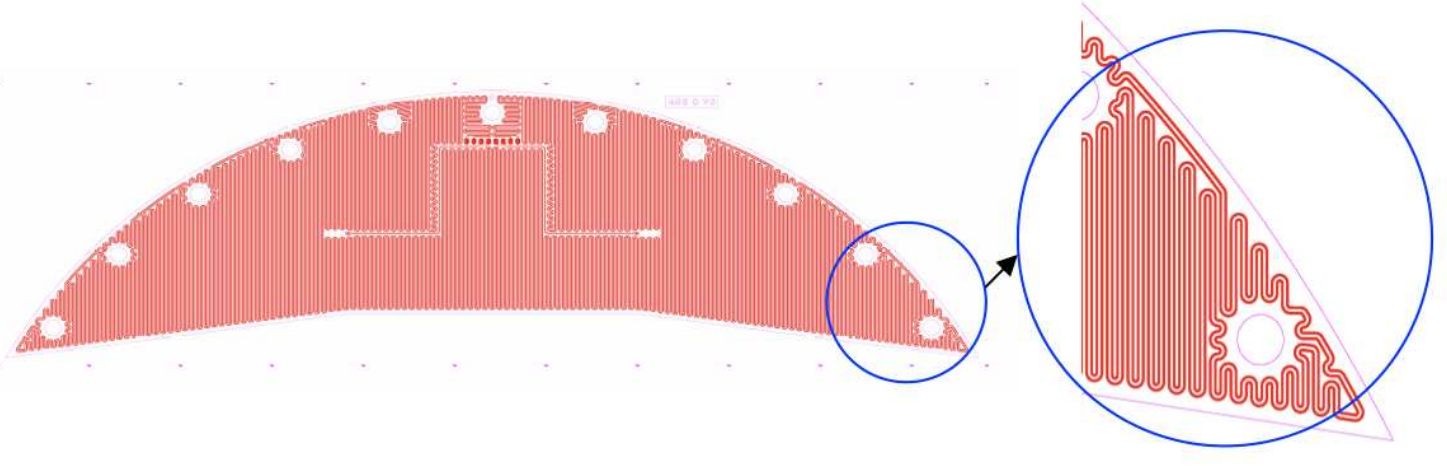

Figure 42. Heater pad for one of the front support in-fill panels (see section 4.1.2) and an enlargement.

These are formed from copper tracks sandwiched between two sheets of polyimide. The copper tracks wind back and forth filling the surface area. The tracks are etched from $8 \mu \mathrm{m}$ foil with track widths between 0.2 and $2 \mathrm{~mm}$ and separations between parallel lines of the order of a few millimetres. There are two separate tracks in parallel to provide some redundancy against failures. Each circuit is designed to produce at least $150 \mathrm{~W} / \mathrm{m}^{2}$ or $300 \mathrm{~W} / \mathrm{m}^{2}$ depending on where it is located. The temperature of the pads is monitored by embedded thermistors which feed-back to the power supplies. The pad temperature is regulated by switching the power with a frequency of the order of $1 \mathrm{~Hz}$ and a rise/fall time of $1 \mathrm{~ms}$. Because of the complicated shapes of components of the end-caps, in particular around the feedthroughs, twelve different types of heater pads are needed, giving a total of 40 heaters for each end-cap, each capable of producing a maximum of the order $100 \mathrm{~W}$ power. An example showing the design of one type of heater pad is shown in figure 42.

\subsubsection{Membranes}

Membranes at the front and rear of the support cylinder (just inside the front and rear supports) serve to complete the Faraday shield and provide a gas seal.

The rear membrane consists of a large sheet of Cu-polyimide extending from the ITE to the STFT, where it wraps over the flange on the OTE. Where the membranes have to bend round corners, tabs are formed by slitting radially the sheet at intervals of a few $\mathrm{cm}$. To prevent tears, the ends of the slits are terminated with small holes. Contact between the tabs and the other $\mathrm{Cu}-$ polyimide surfaces is made by solder bonds. The holes to allow the rear support to be bolted to the support cylinder are sealed with Tempflex $($. These holes were created using a circular punch in association with a large template.

It is expected that when the SCT cools from room temperature to its operating temperature, the membranes will contract by around $300 \mu \mathrm{m}$. To avoid the substantial stresses which will be created in the membranes (around $1000 \mathrm{~N}$ per metre of circumference), "V" creases were placed in the tabs to accommodate the contractions.

At the front, for assembly reasons, there are two membranes: a gas membrane made of plain polyimide and a grounding-and-shielding membrane made of $\mathrm{Cu}$-polyimide (see figure 43 ). 


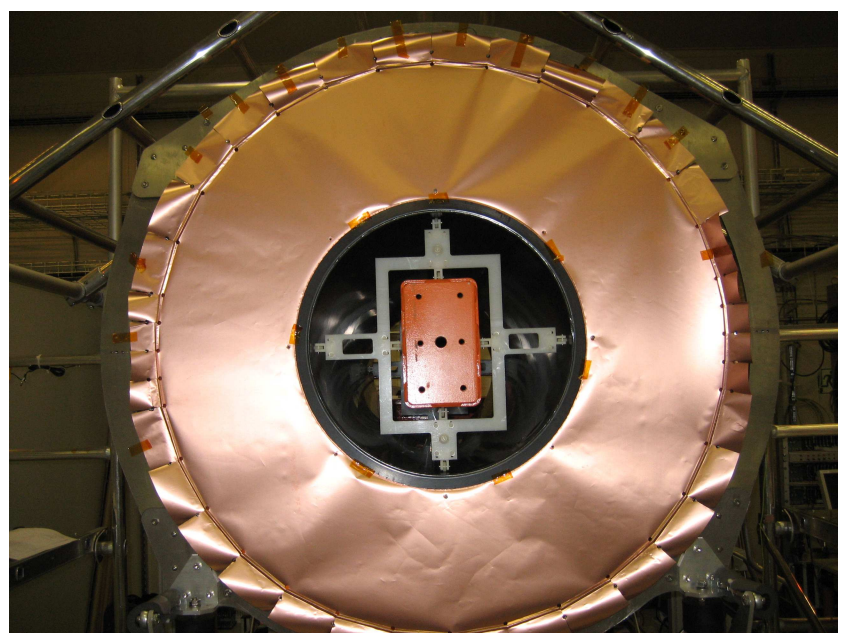

Figure 43. Front grounding-and-shielding membrane ready to be soldered to the OTE. The "V" creases in the tabs can be seen at the outer radii.

\subsubsection{FEA studies}

Early ${ }^{47}$ FEA studies of the mechanical properties of the OTE [47] showed that the effect of a 4 mbar overpressure would produce an increase on the radius of less than $10 \mu \mathrm{m}$, while the effects of cooling from room temperature to the operating temperature would result in a contraction of a few hundreds of microns.

Extensive studies [48] of the design concepts were undertaken using analytic calculations and FEA (using ANSYS). It was found that the heat exchange with the nitrogen purge gas will be negligible; however the gas is responsible for increasing heat flow into the end-cap by convection at the $10 \%$ level. By using high-performance insulating materials such as Pyrogel $\AA$ or Nanopore $\AA$, the heat flow could be reduced further by $\sim 25 \%$ or $\sim 50 \%$ respectively. However, these materials were not used because they are more difficult to handle, would need to be encapsulated and the

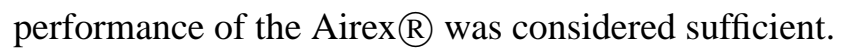

Figure 44 shows a schematic summary of the heat flows associated with sources of heat (heater pads and services) and sources of cooling (LMT cooling and cooling interconnects). The net flow is an inward flux of $\sim 200 \mathrm{~W}$ - this additional heat load will be absorbed by the module evaporative cooling (since this is rated at $10 \mathrm{~kW}$, the additional $200 \mathrm{~W}$ is a small perturbation).

\section{Final assembly and integration with the TRT}

\subsection{Final assembly of the SCT}

The two partial end-cap assemblies were shipped to CERN from their assembly sites, one from The University of Liverpool in the UK and the other from NIKHEF in the Netherlands. By shipping the end-caps before they were inserted into their thermal enclosures, it allowed the end-caps to be supported more robustly. In addition, the same tooling required for this insertion was also used for

\footnotetext{
${ }^{47}$ Corresponding to an aluminium foil outer facesheet rather than $\mathrm{Cu}$-polyimide.
} 


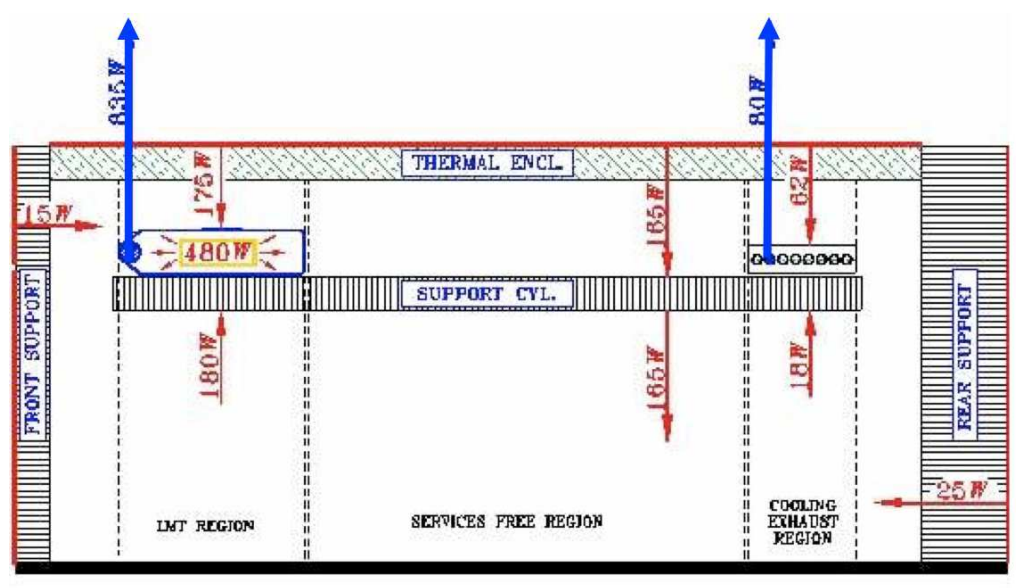

Figure 44. Schematic of heat flows in the end-cap. Thin red arrows show the flow of heat; thick blue arrows show the flow of "cold".

the integration with the TRT. On arrival at the ATLAS surface assembly building, SR1, a thorough reception test was carried out. This included a visual inspection, a repeat of the disk alignment survey as described in section 6.2 and a full electrical connectivity test. Following this, the services

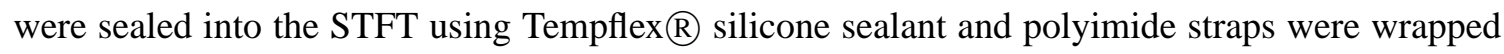
around the services on the cylinder to ensure they did not protrude above the OTE rails.

The final assembly continued in SR1, starting with the addition of the shunt shields, one at each end of each support cylinder. The shunt shields are annular, composite sandwich panels with a core of $4 \mathrm{~mm}$ Airex $\AA$ foam, to which were bonded two layers of $\mathrm{Cu}$-polyimide foil using Araldite $\mathbb{R}$ 2011 epoxy adhesive. The shunt shields cover the outer modules on disks 1 and 9 , and are supported on these disks by fixing them to the module placement pins with electrically insulating nuts. The shunt shields physically protect the modules from movements of the neighbouring gas membranes, as well as shielding the modules from electronic noise. To achieve this shielding, the copper face adjacent to the modules was connected by a tab to the grounding ring on the disk, while the outer copper surface was connected to the ground sheet on the support cylinder.

At this stage each SCT end-cap was still supported by its front and rear stub supports (see section 6.1) on the framework used for the transportation. The first step in preparing for the integration with the TRT [50] was to replace the invar rear stub support (see section 6.1) by the CFRP-composite rear support panel. For this, the weight of the end-cap was transferred to a temporary support ring with adjustable pads resting at twelve points on the support cylinder - see figure 45. This ring eliminated any movement of the support cylinder during the changeover as well as preventing any deformation of the shape of the cylinder. Once supported, the stub support was slid out on rails and the rear support, preassembled with the rear grounding-and-shielding (G\&S) membrane and the rear thermal pad, was assembled to the support cylinder.

For integration with the TRT end-cap, the SCT end-cap and its services (on intermediate supports) had to be transferred from the original assembly support frame to a higher position on a cantilever beam. To achieve this, a jacking system was employed to enable a trolley to be fitted underneath the support frame. The complete system was then slowly manoeuvred along precision 


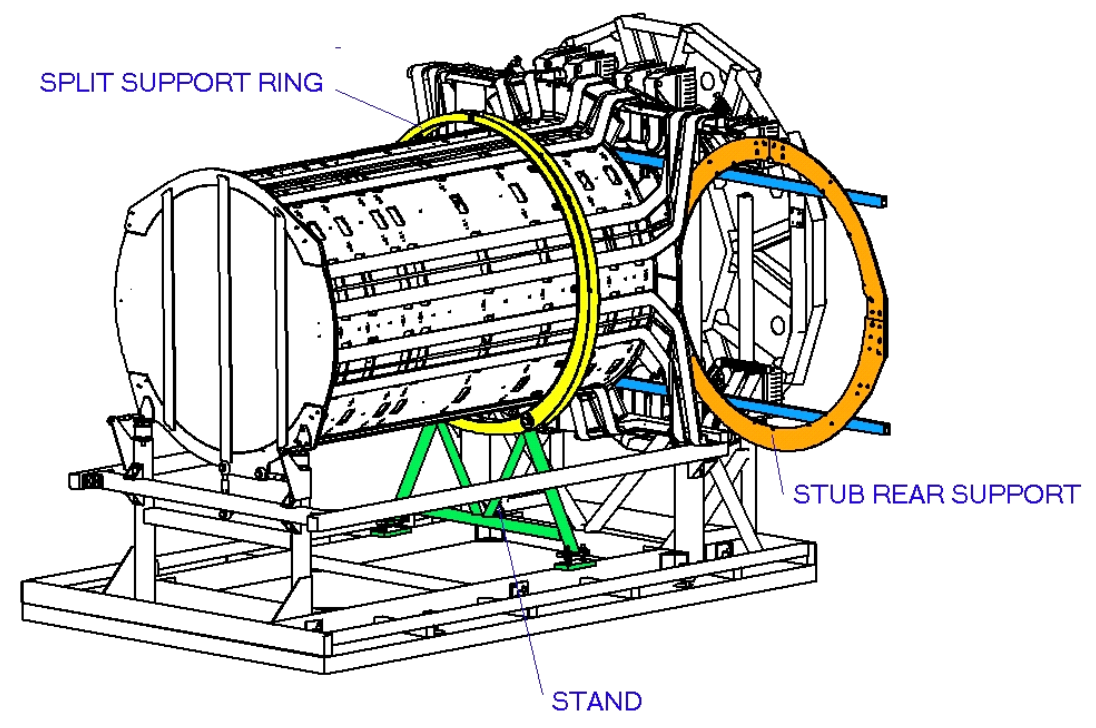

Figure 45. Removal of the rear stub support, during which the end-cap was supported by a temporary support ring.

guide-rails so that the end-cap passed over the cantilever beam. Fine adjustments of the trolley and the beam were undertaken for accurate alignment. The weight of the services was then transferred to the rear of the cantilever beam, allowing some of the support framework to be stripped away.

To insert the ITE, a temporary roller frame was inserted along its inner bore, allowing it to be rolled along the cantilever beam into position. At the rear, the ITE was clamped to the rear support by means of a 4-piece CFRP ring (ITE clamps) and the gas seal (using Tempflex $($ ) ) as well as the grounding and shielding connections were made between the ITE and the rear membrane. The load of the end-cap at the rear support was transferred to the beam using a yoke on the beam which hooked under the protruding "arms" of the rear support.

At the front of the end-cap, the gas and G\&S membranes were attached and sealed (using Tempflex $(R)$, and the seals were made to the ITE at the inner radius of the membranes and the sides of the support cylinder at its outer radius. A temporary front support (TFS) made from invar was attached between the cantilever beam and the front of the support cylinder; the annular front stub support (FSS) had gaps exposing 10 of the 32 support fixing points on the support cylinder (5 on each side) for this operation. As the FSS and TFS used different fixing positions on the support cylinder, it meant the FSS could be removed, leaving the TFS to take the load.

With the full end-cap mass on the cantilever beam, the remainder of the assembly frame was carefully dismantled and the trolley was retracted, leaving the outer circumference of the end-cap clear of obstructions (see figure 46 and also figure 31) in readiness for the OTE, and later the TRT end-cap, to be slid over the SCT. In preparation for the latter, the position of the PPF1 tray holders had to be adjusted to fit inside the envelope of the TRT cable trays.

Before adding the OTE, a further electrical connectivity test was made of all the modules and final inspections and fault finding performed for all the services. The OTE itself was designed to 


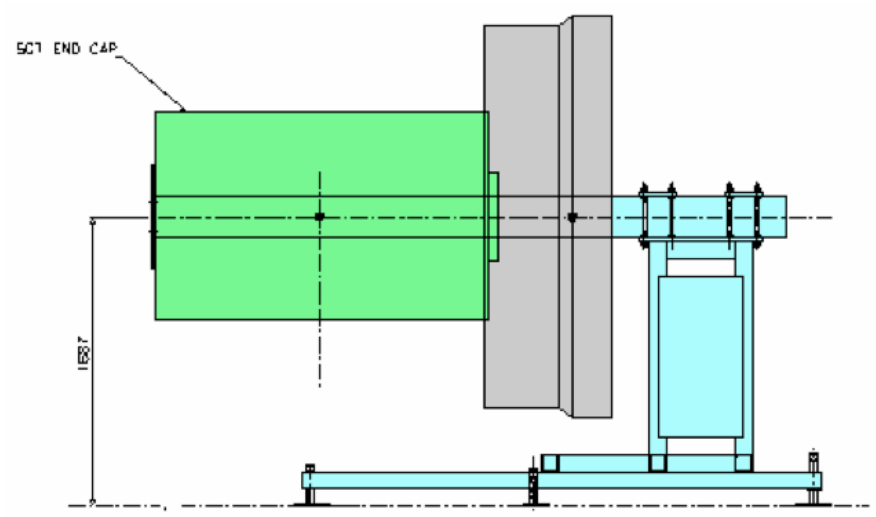

Figure 46. Sketch showing the end-cap held solely on the cantilever beam.

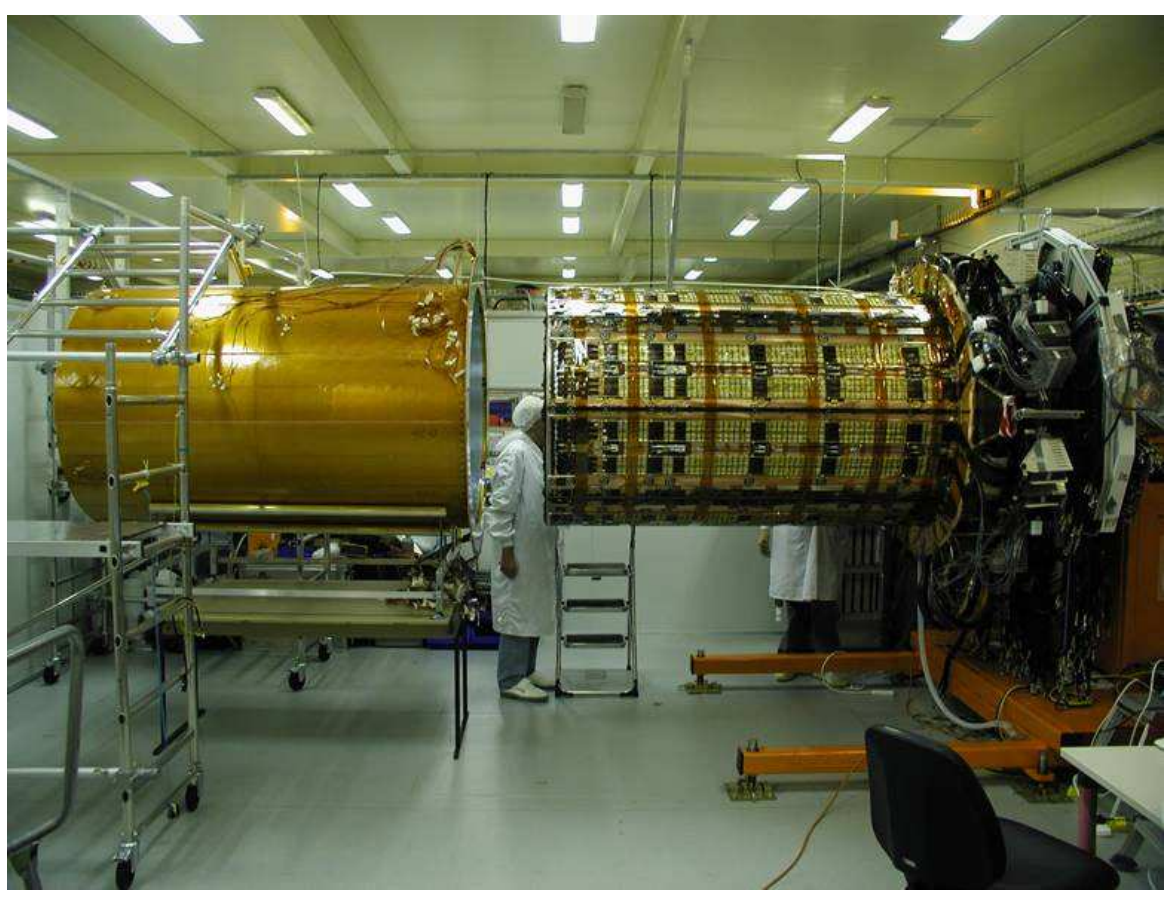

Figure 47. OTE being added to one end-cap.

be located on the rails fixed to the support cylinder. These rails were adjusted prior to mounting the OTE to match the actual inner diameter of the OTE. A simple table-mounted support was used to align coaxially the OTE and SCT EC assembly before sliding on the OTE - see figure 47. Once the OTE was in place, the tabs on the outer edge of the rear G\&S membrane were bent over and soldered to the flange of the OTE. At the front, the front gas membrane was sealed to a flange on the inside of the OTE using Tempflex $®$, then the front G\&S membrane was soldered to the OTE to complete the Faraday cage.

The final addition before the integration stage was of the front section of the radial cable tray (RCT). This segmented aluminium annular plate was fastened to the rear support and extends 


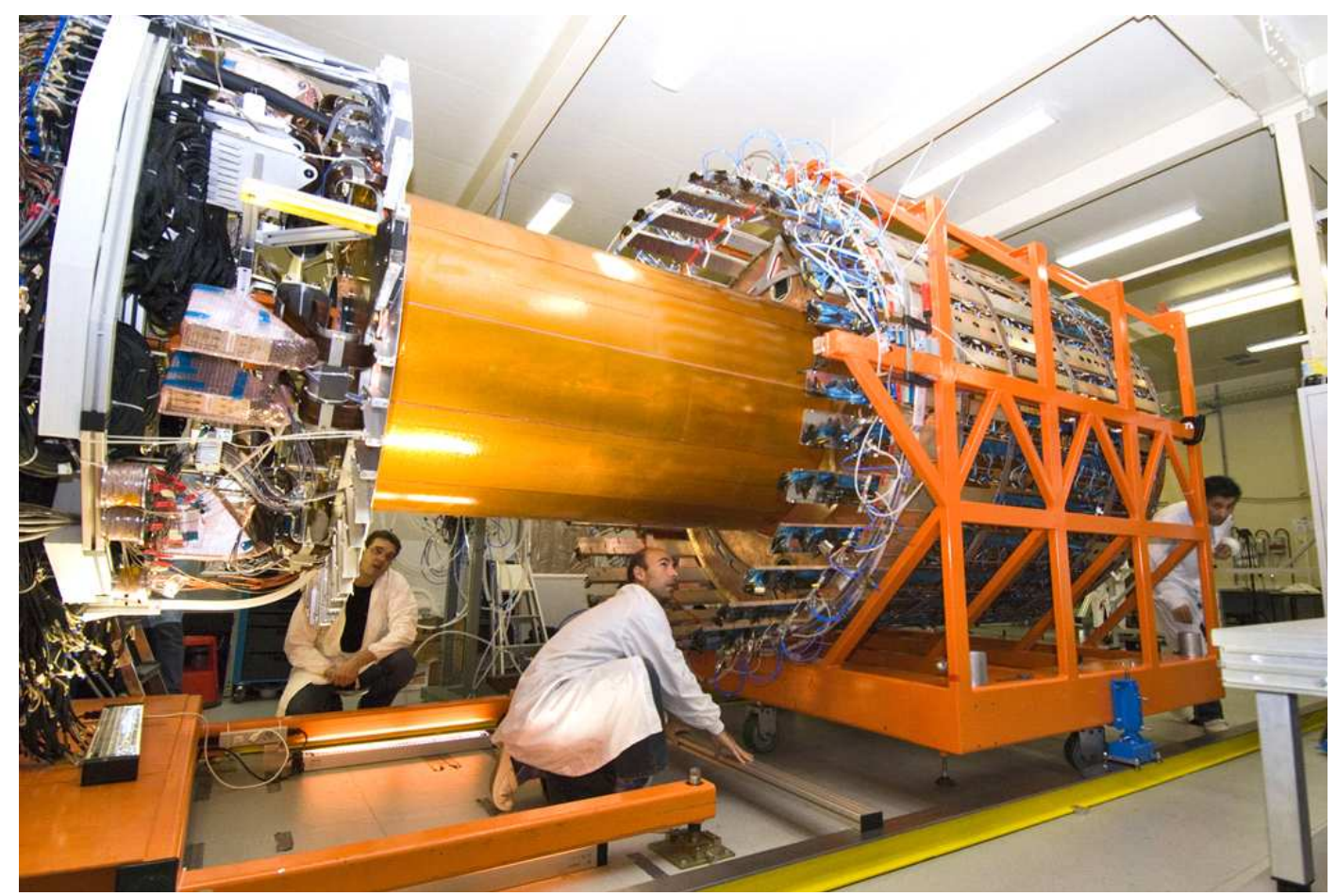

Figure 48. Integration of the TRT and SCT end-caps.

between the STFT and the cryostat, with lidded channels to support and contain the services. In ATLAS, the RCT is used in conjunction with the cryostat cable trays (also with lidded channels) to contain and protect the services up to PPF1.

Following the final sealing of the thermal enclosure, the end-cap was tested for leak-tightness and any remaining gaps were sealed with Tempflex $\AA$. Leak rates of 85 and $360 \mathrm{l} / \mathrm{hr}$ at $1 \mathrm{mbar}$ overpressure were achieved for EC-A and EC-C respectively in SR1 using temporary feedthrough components. For EC-A, no significant localised leaks were found when flushing with argon and using a gas sniffer; for EC-C, some regions of higher leak rate were found but were not accessible for repair. Further electrical tests verified the correct functioning of the modules inside the environment of the thermal enclosure/Faraday cage.

\subsection{TRT integration}

To integrate the SCT and TRT end-caps, guide rails were accurately positioned in front of the SCT, then the trolley carrying the TRT was placed onto these rails. The two detectors were then precisely aligned coaxially using the TRT trolley adjusters. To start the integration, the TRT was slowly pushed up to the SCT and the clearance gap between them checked in several places there turned out to be slightly more than the nominal $6 \mathrm{~mm}$ minimum radial clearance. The TRT EC was then slowly pushed over the SCT. During this operation, the alignment and clearance of the two detectors was continually monitored by observers situated around the assemblies — see figure 48 . 


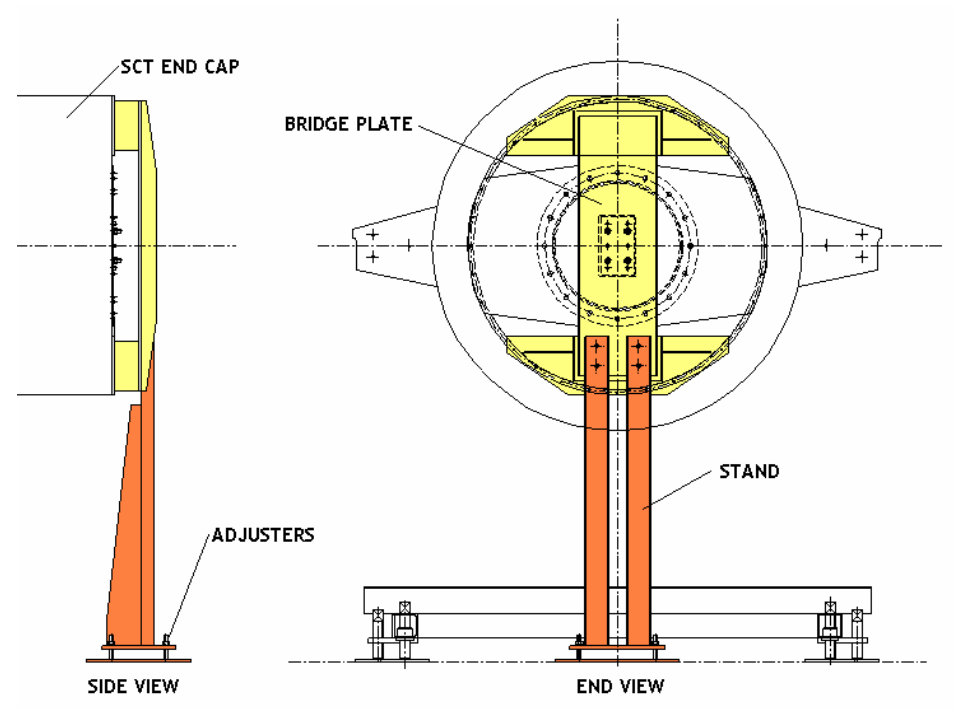

Figure 49. Bridge plate, used to support the front of the SCT end-cap at the top and bottom of the support cylinder.

Once the TRT was fully integrated with the SCT, the bridge plate (see figure 49) was fastened to the 22 exposed fixing points on the support cylinder (11 upper and 11 lower points). The horizontal gap between the plate and the end of the support cylinder allowed access to the TFS fixings and allowed its removal. Subsequently, the front support was able to be inserted into this gap and bolted to the cylinder. The ITE was connected via a flange to the front support, and the roller frame, which was supporting the ITE up to this point, was removed. The support mechanism components (see section 4.1.3) were added to the front support. These were shimmed to just rest on the TRT services support frame (the "squirrel cage") taking the weight of the SCT assembly without changing the relative position of the SCT and TRT. The bridge plate was removed and the in-fill pieces (see section 4.1.2) were added to cover the exposed sections of support cylinder.

The rear support mechanisms were attached to the ends of the rear support and again shimmed to just rest on the squirrel cage. The yoke supporting the rear support was released and removed, transferring the rear load of the SCT to the squirrel cage. Finally, the TRT trolley along with the SCT was retracted from the cantilever beam.

After the integration with the TRT, partial tests of the functionality of each end-cap were carried out. The status is summarised in section 12.1.

\subsection{Survey and dry-out}

The dowels in the support cylinder which define the orientation of the SCT (see section 6.2) were surveyed in the end-cap relative to the temporary rails used for the integration with the TRT. By combining the information from the surveys, the target holder dimensions and the survey of the ID rails, it was possible to determine the transverse position and angle that each end of each end-cap would have when installed in ATLAS [34]. The end-caps were deliberately set about $1.5 \mathrm{~mm}$ low (negative y) because the solenoid field axis is known to be $2.5 \mathrm{~mm}$ low and the rails are expected 


\begin{tabular}{|l|l|l|l|l|}
\hline & EC-A, rear & EC-A, front & EC-C, rear & EC-C, front \\
\hline x offset $(\mathbf{m m})$ & +0.44 & +0.07 & +0.43 & -0.33 \\
\hline y offset $(\mathbf{m m})$ & -1.09 & -1.92 & -1.34 & -1.79 \\
\hline Rotation $(\mathbf{m r a d})$ & +0.29 & -0.21 & +0.67 & +0.52 \\
\hline
\end{tabular}

Table 7. Calculated $x-y$ positions of the two ends of each end-cap when installed on the inner detector rails. Positions are relative to the $\mathrm{IWV}^{48}$ coordinate system and do not include the expected rail sag.

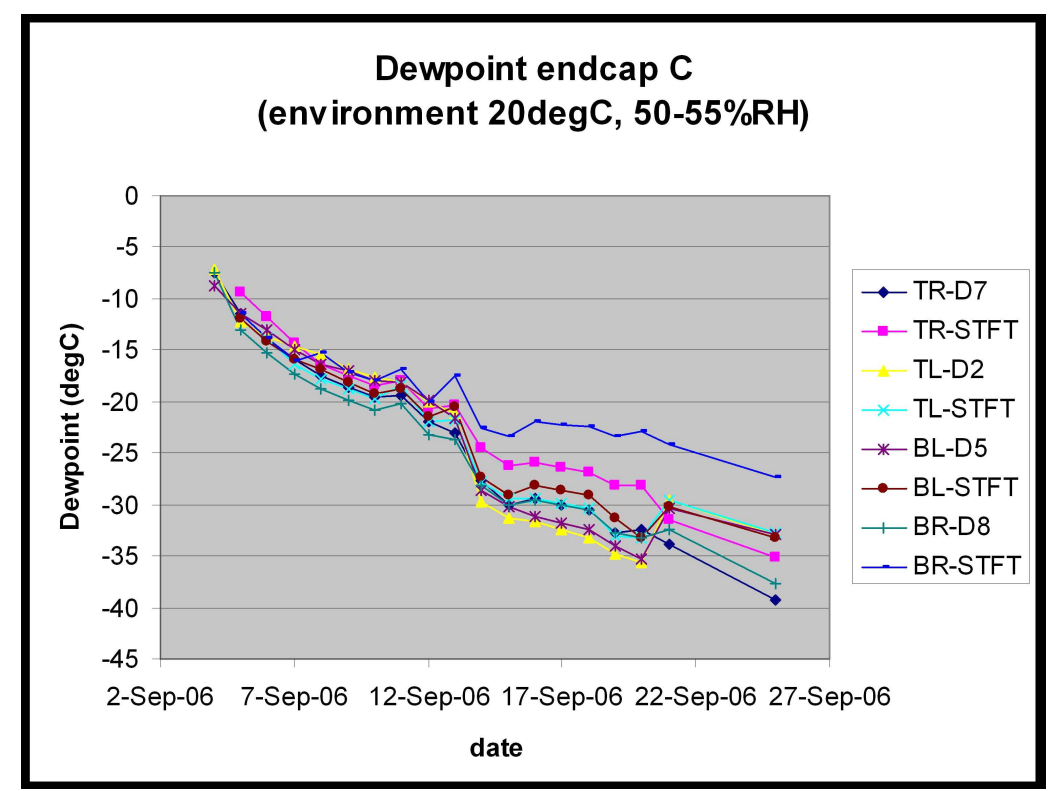

Figure 50. Dew-point measured at various points inside EC-C while it was flushed with dry $\mathrm{N}_{2}$.

to sag by $1 \mathrm{~mm}$ when loaded with the weight of the inner detector. It was necessary to re-adjust the end-cap positions with shims after the first survey in order to reach the final positions reported in table 1. Since ATLAS itself has an upward rebound, the alignment with respect to the beam-line is still to be determined.

It is essential that the SCT can be dried sufficiently to ensure there is no condensation inside. Figure 50 shows the dew-point measured inside EC-C while it was flushed with dry $\mathrm{N}_{2}$ during a test. After four weeks, the dew-point was below the expected internal temperature of the SCT and still falling.

\section{Installation into ATLAS}

Having integrated the SCT and TRT, the final phase of the engineering project was the transportation of the end-cap to ATLAS, the insertion into the cryostat and the connection of all the services.

\footnotetext{
${ }^{48}$ Inner Warm Vessel — the inner wall of the cryostat containing the solenoid.
} 


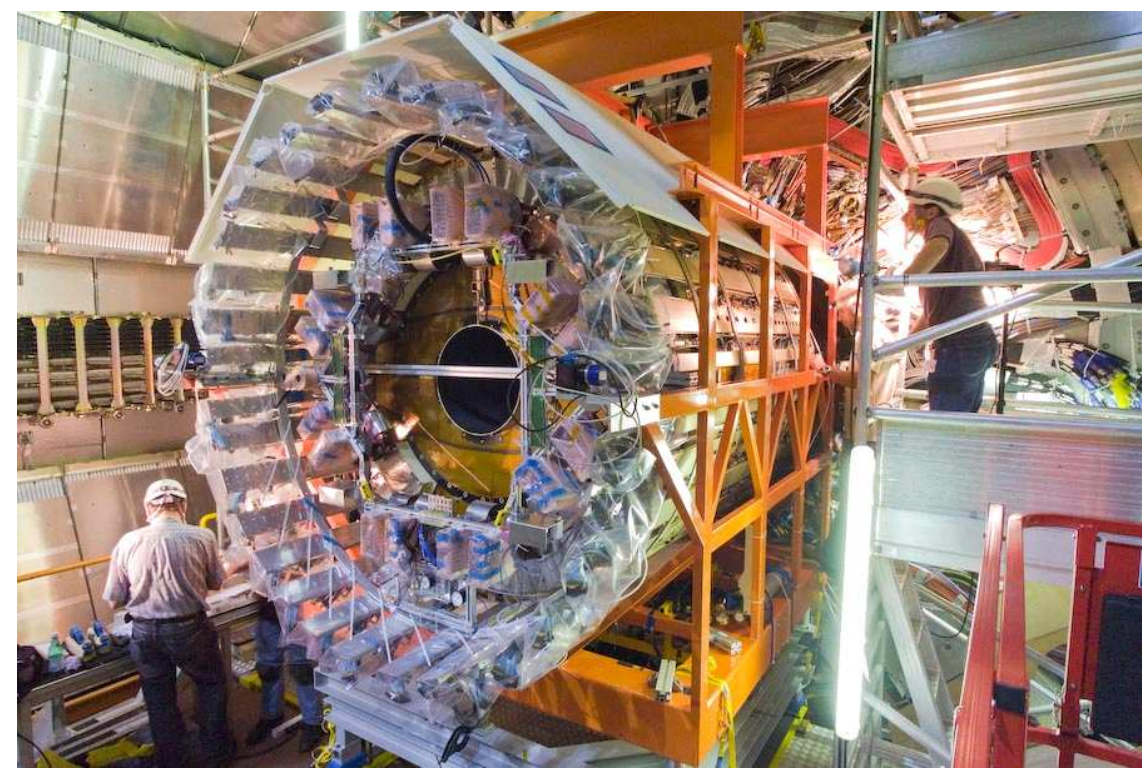

Figure 51. Installation of EC-A into ATLAS.

\subsection{Transport and insertion into ATLAS}

The movement of the end-caps to ATLAS was more delicate than the transportation to CERN, since the SCT was now supported by the CFRP front and rear supports, as opposed to the more rigid metal stub supports (see section 6.1). The maximum dynamic acceleration was specified to be less than $0.1 \mathrm{~g}$ in the range 1 to $100 \mathrm{~Hz}$ and the maximum equivalent static acceleration to be smaller than $0.2 \mathrm{~g}$ in any direction [49].

Clamps were mounted to restrain the support ends vertically and rigid bar supports were added to prevent longitudinal motion. Capacitive accelerometers were installed onto the SCT in the longitudinal and vertical direction in order to monitor the accelerations. A tilt-meter was added to ensure that a maximum tilt of $1^{\circ}$ about the horizontal axis was not exceeded. Each end-cap was placed in a wooden box and wheeled out of SR1, with rails over the uneven parts of floor. To move the end-cap the $100 \mathrm{~m}$ to the ATLAS service building, mobile cranes were used in order to minimise the shock.

Once inside the service building, the end-cap was lowered down the shaft using the service building cranes. In the pit, the end-cap was positioned on a cradle and rotated $90^{\circ}$ to be inline with the cryostat bore. Then the end-cap trolley was moved on rails towards the cryostat bore. Having aligned the rails, the end-cap was inserted using a push-pull mechanism — see figure 51. This single drive ensured that on both sides of the end-cap, the movements were equal and no shear was introduced. Wire length meters were used to monitor the difference in movement between the two sides.

Because the inner detector barrel was outside its envelope longitudinally (in z) and to prevent damaging the services of the barrel, a contact sensors made of thin copper sheets were placed between the barrel and end-cap (see figure 52). This sheet was connected to an electrical circuit to detect when the assemblies made contact and hence avoid additional forces which might lead to 


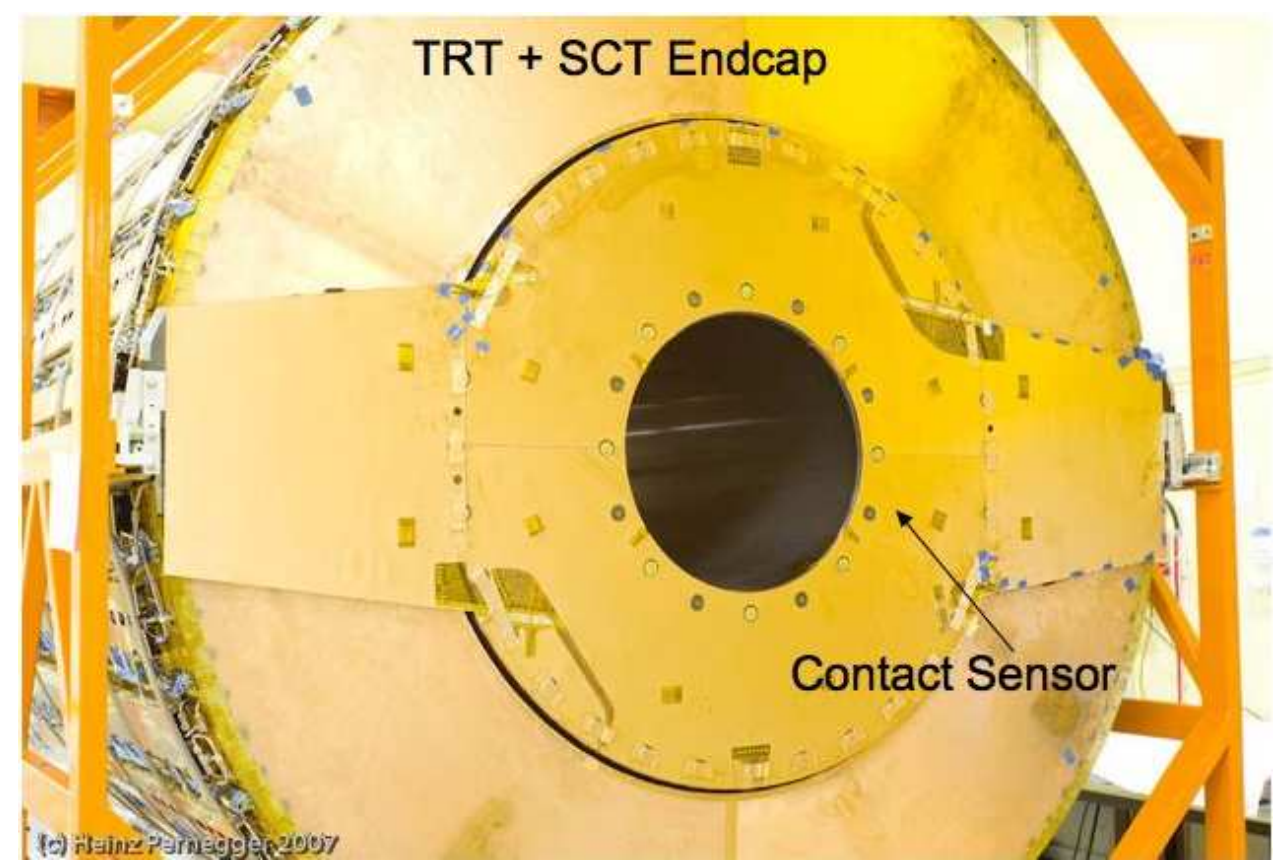

Figure 52. Contact sensor on the SCT end-cap: to detect (electrical) contact with the SCT barrel when the end-caps were installed.

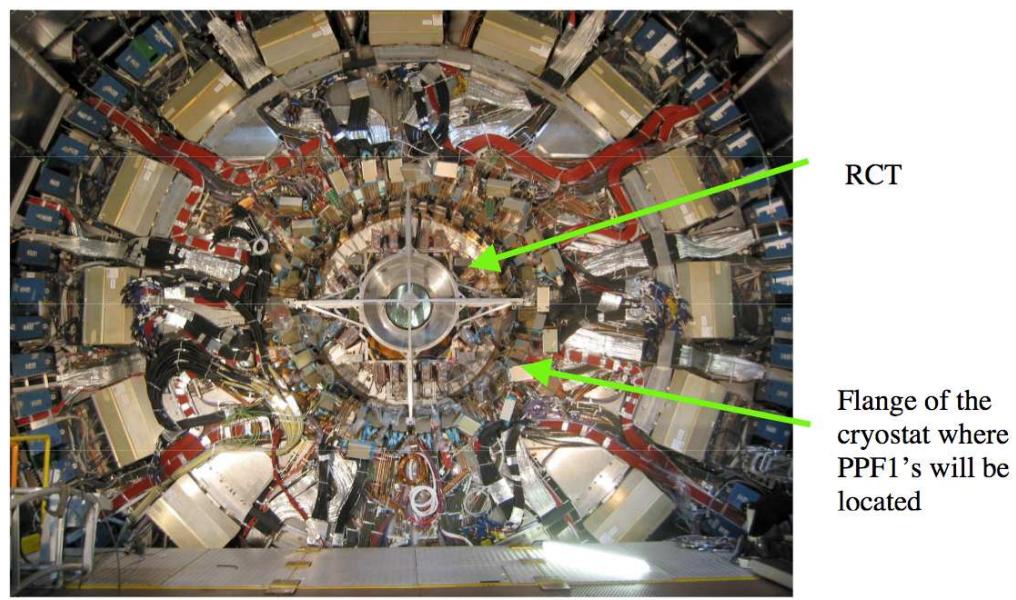

Figure 53. End-cap installed in ATLAS before the completion of services installation.

damage. For both end-caps, contact was made approximately $5 \mathrm{~mm}$ before their nominal positions in z. EC-A is $3 \mathrm{~mm}$ longer than its nominal length, half of this excess resulting from a longer OTE.

\subsection{Installation of services}

The final step was the routing and connection of the SCT services at the PPF1 patch panels near the corner of the cryostat - see figure 53. Table 8 summarises the services for each end-cap and the numbers and types of patch panels. A schematic of the PPF1 layout is shown in figure 54. All 


\begin{tabular}{|c|c|c|}
\hline Services & Number & Patch Panel \\
\hline PPF1 housings and cable trays & 18 & 16 for LMT's, 2 for TE Heater wires - see figure 54 \\
\hline LMT monophase cooling loops & 16 & $\begin{array}{l}\text { Divided over } 8 \text { electrical and } 8 \text { combined electrical } \\
\text { and optical patch panels }\end{array}$ \\
\hline Low-mass tapes (LMTs) & 988 & \\
\hline $\begin{array}{l}\text { Readout optical ribbons: data } \\
\text { and TTC }\end{array}$ & $177+89$ & $\begin{array}{l}\text { Divided over } 8 \text { combined electrical and optical patch } \\
\text { panels }\end{array}$ \\
\hline FSI optical ribbons & 45 & $\begin{array}{l}\text { Run through } 4 \text { combined electrical and optical patch } \\
\text { panels; spliced in splice-boxes }{ }^{49}\end{array}$ \\
\hline Heat exchanger assemblies & 36 & $\begin{array}{l}\text { Divided over } 4 \text { cooling and } 4 \text { combined cooling and } \\
\text { DCS patch panels }\end{array}$ \\
\hline DCS cables & 40 & $\begin{array}{l}\text { Divided over } 4 \text { combined cooling and DCS patch } \\
\text { panels }\end{array}$ \\
\hline Grounding and shielding clamps & 16 & $\begin{array}{l}\text { Connected at } 16 \text { places on the radial cable tray } \\
\text { (RCT) }\end{array}$ \\
\hline Heater pad cables & 36 & $\begin{array}{l}\text { Divided over } 2 \text { combined heater pad and nitrogen } \\
\text { patch panels }\end{array}$ \\
\hline Nitrogen tubes & 5 & \\
\hline
\end{tabular}

Table 8. Summary of the external services and their patch panels (PPF1) for one SCT end-cap.

the services from PPF1 to the racks were preinstalled and tested. Most of the services were routed and connected layer by layer, with each layer being tested as soon as it was complete, because each subsequent layer blocked access to previous layers.

The first step in the installation was the connection of the cryostat cable trays (CCT's) and the $\mathrm{Cu}$-polyimide shielding, followed by the LMT monophase $\mathrm{C}_{4} \mathrm{~F}_{10}$ cooling loops. The cooling loops provide the cooling for the LMTs outside the SCT and for the power cables to which the LMTs connect. The next steps were routing of the optical fibre ribbons and installation of the electrical PPF1 tray housings for LMT connections. The FSI ribbons were routed to the splice box on the cryostat flange, and the readout ribbons were installed in the patch panels. Connection and testing of the optical fibres required a significant synchronization with the LMT connection. Half of the electrical PPF1 positions also support optical fibres, and in these cases, the LMTs block access to the readout fibre connections. Optical fibres needed to be installed before the LMTs sharing the same position, but in order to test the optical fibres, their corresponding LMTs, located in the previous quadrant, had to be installed already.

Following the installation of the PPF1 tray housings, the LMTs were connected (see figure 55). Each quadrant has four PPF1 tray housings, with each housing holding nine trays, one per disk. Each tray is equipped with up to nine LMTs. Connection tests were performed in the electronics caverns which house the power-supplies, using a standalone test system to measure the resistances of all the lines. Problems with the connections or cables were fixed immediately before

\footnotetext{
${ }^{49}$ Located in the crack between the barrel and end-cap calorimeters.
} 


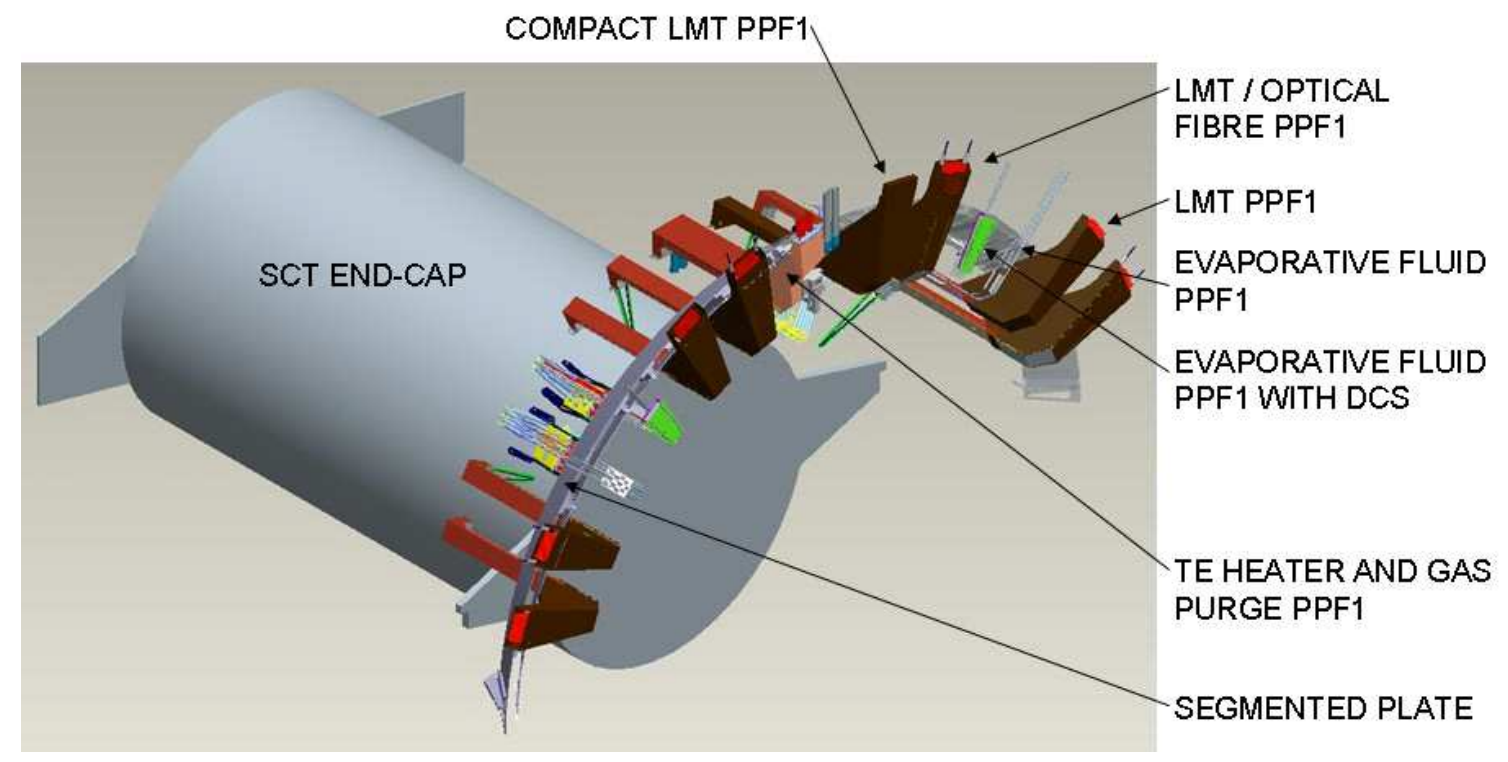

Figure 54. Schematic showing one half of the PPF1 patch panels.

moving to the next layer. In total, 157 problems were registered with the LMT installation. These included bad connections at PPF1 and incorrectly mapped cables, which were easily resolved, as well as several problems downstream of PPF1, some of which required repairs. All repairs were successful except for one, where an LMT was found shorted to ground in an inaccessible region, therefore was beyond repair. After the installation of each complete housing, the LMTs were wrapped with aluminium foil together with the LMT monophase cooling loops, the cable tray lids were added and the Cu-polyimide foils closed providing shielding for the PPF1.

All the readout ribbons for one quadrant were connected prior to testing. The connection sequence for ribbons and LMTs had to take into account that while the LMTs bringing power to modules exit the end-caps in the same quadrant as the modules, the optical ribbons leaving a particular quadrant of a disk are connected to modules in the neighbouring quadrant. Once the electrical connections were made, the optical connection could be tested in the absence of cooling, by only powering each module for about 10 seconds to avoid over-heating. To test the readout optical ribbons, the PIN currents were measured and a super-fast RX threshold scan was performed.

Next, the heat exchanger (HEX) assemblies were installed (see section 1.5 and figure 56). Originally the heaters for the evaporative cooling exhaust lines should have been installed together with the HEX assemblies, but due to electrical problems with the heater connectors, it was decided to redesign the system and move the heaters to a more accessible position on the cryostat flange to allow for future maintenance. The HEX assemblies were joined to the connectors at the end of the on-cylinder cooling circuits at the point where they leave the end-cap. Specially made connectors were used with PEEK washers to make electrical breaks between the on-cylinder pipes and the HEX assembly. Each circuit was leak tested before the adjacent HEX was installed. To complete the cooling circuits, the heaters were connected to the exhausts of the HEX's by short pipes. After completion of all the circuits in a quadrant, leak tests of the complete evaporative lines 


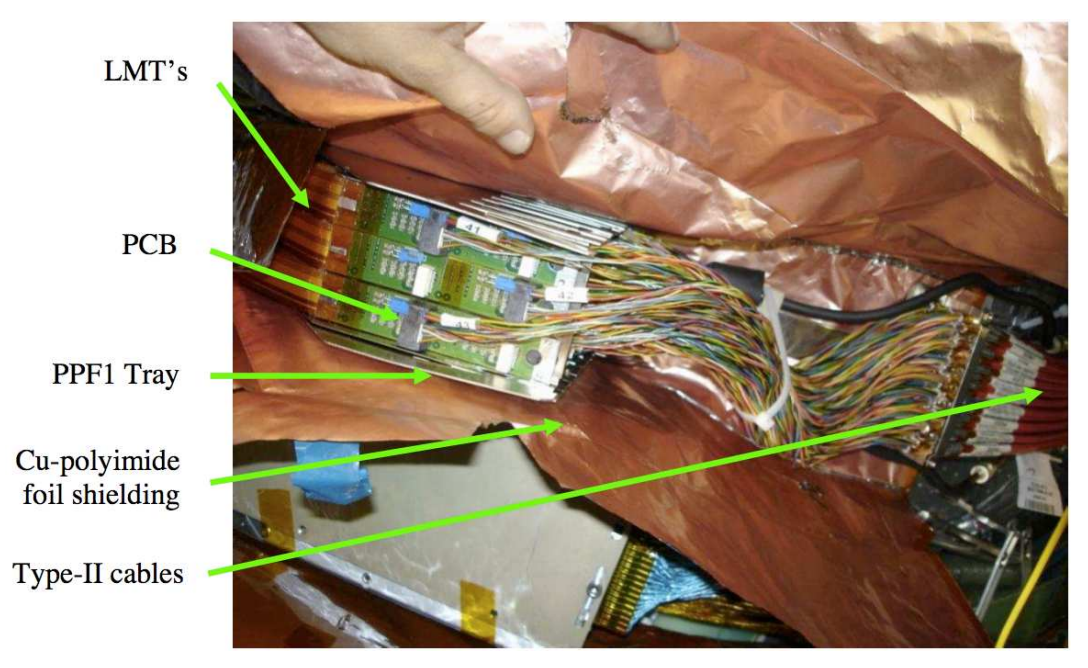

Figure 55. LMTs attached to their trays in an electrical PPF1 housing, before it is closed up.

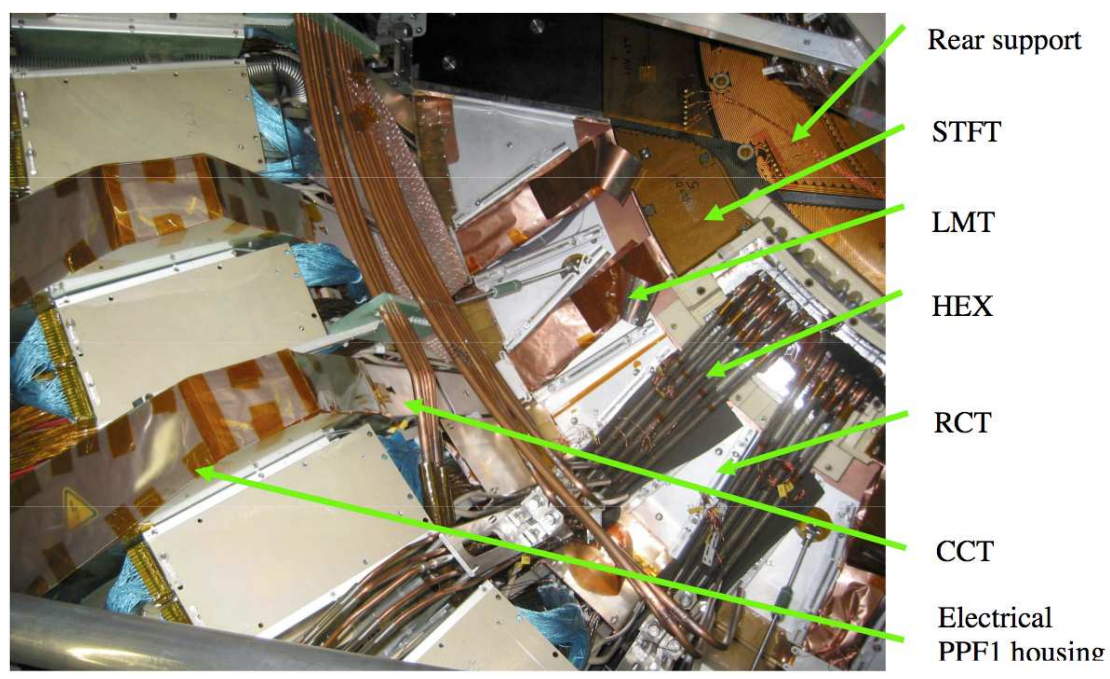

Figure 56. View of services, in particular the cooling circuits and the LMTs, at the rear of the end-cap and inside the cryostat. At this stage, the feed-through boxes were yet to be added to seal the STFT and the rear sections of the RCT are not in place.

were performed. Feed-through boxes were then added and sealed to close the end-cap thermal enclosure at the STFT, and then all the pipes were insulated.

With all the services installed, the rear sections of the RCT were added to support the radial services. The final step in the installation was the leak-testing of the thermal enclosure volume, followed by the connection and testing of the DCS cables, heater pad cables and the nitrogen lines. 


\section{Grounding and shielding}

\subsection{Concepts}

The strategy for the grounding and shielding [51] is that all conductors not used for carrying currents to/from the detector must be connected by a low-impedance path to the ATLAS main ground - this includes components on the disks, support cylinder and thermal enclosures. Where possible, loops are avoided to reduce the noise picked up on signal lines.

The end-cap module has a number of existing features to limit the stray capacitance of the detector backplane to the cooling circuit. Primarily, the backplane AC-referencing conductor is routed to a large bypass capacitor on the hybrid, which couples the backplane to the front-end analogue ground. The capacitor is of order ten times larger than the detector stray capacitance to the cooling circuit. The module is separated from the cooling block by electrically insulating thermal grease $;^{50}$ the cooing block itself is well connected electrically to the cooling circuit by the solder joint. In order to limit noise currents in the front-end, the cooling pipe is referenced by a DC connection to the module at the power-supply module entry region and a DC connection from analogue ground on the module to the cooling tube is implemented (more details can be found in [51]). The hybrids are connected to the grounds on the power tapes. To prevent the timing, trigger and control (TTC) redundancy links from generating excess noise in the front-ends, there are very low-impedance connections for the digital return signal from one module to its neighbour.

The cooling pipes are connected to the grounding foils on the disks, which in turn are connected to the ground sheet on the support cylinder. Likewise all the metallic components inside the OTE are connected to the ground sheet (see section 4.2), which provides the principle grounding path out of the end-cap to the radial cable tray (RCT).

To shield the front-end electronics from external pick-up noise, the end-cap is enclosed in a Faraday shield formed from the Cu-polyimide foil on the OTE and ITE and the front and rear membranes. This shield also connects to the RCT (see section 9.1). Originally it had been hoped to use aluminium facesheets to reduce the number of radiation lengths; however because of the difficulties of making reliable low-resistance connections with aluminium, copper was chosen since this allows strong physical bonds of low resistance to be made very easily. Sheets of Cu-polyimide were connected using a "bridging" technique explained in section 8.4.1 and illustrated in figure 57 . To facilitate the handling of the copper foil strip ( $3 \mathrm{~mm}$ wide) used to form the "bridge", the foil was cut into sections $\mathrm{O}(1) \mathrm{cm}$ long and joints were made with $\mathrm{O}(1) \mathrm{cm}$ gaps between each length of foil along the joint. At the services feedthrough (STFT), gaps allow services to pass through. As a rule of thumb, an attempt was made to limit apertures to less than $1 \mathrm{~cm} \times 10 \mathrm{~cm}$.

The RCT is a significant node in the grounding of the end-cap; this is ensured by its good conductivity. There is an insulating break at one azimuthal position in the annulus to avoid the forces associated with a solenoid quench ${ }^{51}$ (see section 1.3). However, to ensure that good connectivity is maintained around the annulus for high-frequency currents, the break is made with a set of capacitors. ${ }^{52}$

\footnotetext{
${ }^{50}$ DC340, manufactured by Dow Corning.

${ }^{51}$ Without the break, the longitudinal force would be $\mathrm{O}(1600) \mathrm{N}$.

${ }^{52}$ Twelve $10 \mu \mathrm{F}$ ECJ-CV50J106M capacitors, manufactured by Panasonic.
} 


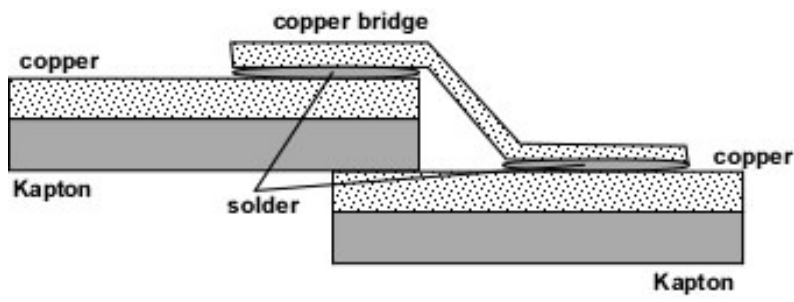

Figure 57. "Bridging" technique for solder connection between two sheets of Cu-polyimide.

The RCT is electrically connected to the cryostat cable trays (CCT's). To ensure good connections, the cable trays were treated with Alochrome 1200 and fingerstock ${ }^{53}$ was inserted in the gaps to ensure penetration of surface oxide layers. All services, including the monophase LMT cooling pipes are referenced to the CCT's, which are connected in turn to the PPF1's. Finally the PPF1's are bolted to the segmented plate located at the flange of the cryostat (see figure 54 - this plate is robustly connected to the inner detector ground, which in turn is connected to the main ATLAS ground. To avoid noise transmission from outside to the end-cap, the module evaporative cooling circuits are isolated at the STFT, while the monophase cooling pipes are isolated in the vicinity of the PPF1's.

\subsection{Measurements}

To check the correct implementation of the grounding scheme, extensive measurements were made to ensure that intended connections were indeed of low resistances and no unwanted electrical paths had been created inadvertently. DC measurements of various items used in the assembly of the two end-caps were made in a systematic way in the SR1 surface building at CERN so that any rectifications could be undertaken before the end-caps were installed in ATLAS.

Two measurement techniques were used:

1. A standard multi-meter was used to measure resistance with a precision of $0.1-0.2 \Omega$. Since a multi-meter functions with a very small voltage, this method presented a low risk for devices built with semiconductors.

2. A current source set with a limit of $3 \mathrm{~A}$ combined with a very precise voltage measurement ${ }^{54}$ enabled resistances to be determined down to $10 \mu \Omega$ (impossible with the other technique). This method, unsuitable for sensitive devices, was used to ensure good connectivity for the outer Faraday shield, in particular the connections within and to the OTE and ITE.

The common-sense criteria used when checking the various connections were that conducting paths should have resistances less than $0.2 \Omega$ (the limit of precision of the multi-meter); while insulating paths should have resistances in excess of $1 \mathrm{M} \Omega$.

The measurements were made at various stages of the End-Cap assembly in SR1. Following the reception of the end-caps from Liverpool and NIKHEF, it was found that pipe brackets on

\footnotetext{
${ }^{53}$ Supplied by Laird Technologies.

${ }^{54}$ Using a Keithly 2010 instrument.
} 


\begin{tabular}{|l|l|}
\hline Test & Noise $($ enc) $( \pm \mathbf{6 0})$ \\
\hline Liverpool: After assembly & 1582 \\
\hline SR1: Single discs powered, thermal enclosure on & 1555 \\
\hline SR1: All discs powered, thermal enclosure on & 1548 \\
\hline SR1: All discs powered, thermal enclosure heaters on & 1537 \\
\hline SR1: After insertion into TRT & 1522 \\
\hline
\end{tabular}

Table 9. Noise measured for outer modules in a phi-wedge of EC-C, corrected to $0^{\circ} \mathrm{C}$.

the support cylinder were floating, therefore connections to the ground sheet were made. Before integration with the end-cap, the OTE, ITE, shunt shields, front and rear support assemblies were subjected to a large number of measurements. The connectivity between the different sheets of $\mathrm{Cu}$ polyimide (resulting from the bridging technique, described in the previous section) within the OTE and ITE was much better than $1 \mathrm{~m} \Omega$. However it was found to be necessary to add conductive tabs connected with conducting glue to ensure the electrical connectivity of the aluminised polyimide sheets. To ensure the isolation of the SCT from the TRT rails, the carbon-loaded PEEK inserts in the support mechanisms (see 4.1.3) had to be replaced by unloaded PEEK ones.

During the insertion of SCT EC-C into the TRT end-cap, a short via the RCT was detected. This was cured by adding a $200 \mu \mathrm{m}$ sheet of G10 to the RCT surface. The same precaution was taken for EC-A.

After the insertion of EC-C into ATLAS, a connection of $2 \mathrm{k} \Omega$ between the SCT end-cap and the SCT barrel was detected. The short was between one of the front support mechanisms (see 4.1.3) and the SCT barrel. By inserting an endoscope between the cryostat and the end-cap, it was found that the short was due to a $\mathrm{CO}_{2}$ pipe. The pipe was moved in situ and some insulation inserted. Also, to ensure that the end-caps are complexly isolated from all other components, an alarm monitor ${ }^{55}$ detects currents as small as $10 \mu \mathrm{A}$ flowing through a fault path.

\section{Status of the end-caps}

From arrival at CERN to readiness to be installed in ATLAS took around one year.

\subsection{Status of the hardware}

To test possible interference in various configurations, in particular after insertion into the TRT, a phi-wedge of 111 modules was cooled and tested. The measurements were undertaken with prototype cables and readout fibres, using a clock speed of $40 \mathrm{MHz}$ and various trigger rates. These prototypes were not as long as the final ones to be used in ATLAS, and the readout fibres were not radiation-hard, whereas the final ones will be. The comparisons with earlier measurements are show in table 9. It can be seen that the performance is stable and not unduly affected by the environment.

At the time of writing, both end-caps have had all their electrical and optical services connected. The electrical connections are all checked by resistance measurements from the power

\footnotetext{
${ }^{55}$ Based on the Integrated Parametric Current Transformer, manufactured by Bergoz Instrumentation.
} 
supply crates, and functionality tests have been undertaken by powering the modules for around 10 seconds at a time, to verify the optical connections. In addition, the bias voltage has been applied to all modules, and the leakage current as a function of voltage has been checked. However, more complete measurements of the module performance will only be made after the cooling services have been installed.

To date, there is one module in EC-C out of the 1976 in both end-caps which cannot be readout due to a short on the VCSEL (vertical-cavity surface-emitting laser) control line. Efforts are being made to recover this module. There are 27 (1) and 15 (2) dead data (TTC) links in EC-A and EC-C respectively. There is no loss of functionality because of the built-in redundancy.

With the closure of the feedthrough, the leak rates in EC-A and EC-C at 1 mbar overpressure are $45 \mathrm{l} / \mathrm{hr}$ and $390 \mathrm{l} / \mathrm{hr}$ respectively. The end-caps have been dried out with an input flow rate of $650 \mathrm{l} / \mathrm{h}$, and by adjusting the valves on the exhaust line to maintain an overpressure. These leak rates exceed the stringent specification of $25 \mathrm{l} / \mathrm{hr}$. Nitrogen which leaks out to the inner detector volume may be a problem for the TRT, which is very sensitive to this gas. In this respect, the end-caps are not as big a problem as the SCT barrel, which has a much larger leak rate and cannot achieve a 1 mbar overpressure even with a flow of $2000 \mathrm{l} / \mathrm{hr}$. Therefore, it may be necessary to clean the recirculated Xenon-based active gas in the TRT more carefully or change the environmental gas surrounding the SCT and TRT. Once the end-caps are dry, the flow rate will be reduced, corresponding to an overpressure of 0.4 mbar. This should halve the leak rate and reduce the likelihood of the leak rate increasing with time.

Following the initial submission of this paper, leaks were detected in two of the evaporative cooling circuits for disk 9 of EC-C. One circuit has a leak of $25 \mathrm{mbar} / \mathrm{hr}$ at 7 bar of Helium — this is just above the specification and may be tolerable; the other has a leak rate of $1 \mathrm{mbar} / \mathrm{s}$ and this circuit cannot be used unless it can be repaired. The exact locations of the leaks are not known but are probably between PPF0 and the STFT (feedthrough) - access to this region is close to impossible. Due to the ATLAS schedule, the end-caps have had to be sealed up and although repair scenarios are still being investigated, there is a significant likelihood that 13 modules in one quadrant of disk 9C will not be able to be operated. This also means that one of the LMT cooling circuits will be inoperable, although cooling will be provided by the circuits associated with disks 7 and 8.

\subsection{Mass estimates}

It is important to estimate the mass of the detector to ensure that it conforms well to the expectations and does not exceed the limits calculated for the support structure. The original design estimate of the mass was $168 \mathrm{~kg}$. It is calculated that $30 \%$ of the load is on the front supports, while the rest is on the rear supports. In the structural analysis, a safety factor of two was used for the material properties, and there was a further safety factor of at least $30 \%$ in the predicted stresses. After the support structure was manufactured, it was proof-tested to more than 1.5 of the original working load (277 kg of lead blankets were draped over the structure). After the front and rear supports were modified to take the survey targets, they were re-tested. Since the mass budget had increased, so they were re-tested with a load of $300 \mathrm{~kg}$ using a dummy structure bolted between the supports.

Furthermore, it is important to know the mass distributions accurately to understand the effects of multiple-scattering, nuclear interactions, bremsstrahlung and pair creation in the detector and to 


\begin{tabular}{|l|l|}
\hline Components & Mass (kg) \\
\hline Modules & 23.7 \\
\hline Disks & 32.8 \\
\hline Support cylinder, associated services and OTE & 57.4 \\
\hline Other support structures (front and rear supports, ITE) & 22.8 \\
\hline Services between STFT and cryostat, including RCT & 41.2 \\
\hline Services on cryostat, including cryostat cable trays & 46.8 \\
\hline PPF1s & 34.5 \\
\hline Total & 259.2 \\
\hline
\end{tabular}

Table 10. Bottom-up estimates of masses of end-cap components for one end-cap.

ensure the correct representation in the Monte Carlo simulation for physics studies. To ensure an accurate transfer of the energy scale from the inner detector to the electromagnetic calorimeter, it is desirable to understand the material distribution at the level of $1 \%$ (of its value). This is very challenging, although this is most critical at the inner radii. Components of the end-cap have been systematically weighed, although this has been harder for those components at higher radii, where significant amounts of Tempflex $₫$ sealant have been required, additional fastenings have been added and the masses of cables and their wrappings have been less easy to control. Nevertheless, the estimates for the disks before the addition of modules (which are well described themselves) from the bottom-up approach agree with the weighed values to $1.4 \%$, and even then the simulated values are adjusted to reproduce the total weights. At the same time, care has been taken to understand the composition of the materials used to estimate correctly the radiation lengths. The estimates of the various components are given in table 10 .

Although the estimated mass of the end-caps has grown, this is primarily due to external services and the mass which is estimated to be supported by the front and rear supports is $178 \mathrm{~kg}$ - to be compared with the original design estimate of $168 \mathrm{~kg}$ (above). An effort was made to weigh the SCT end-caps while they were inside the TRT (the TRT had been weighed previously). Understanding what additional items were included in the measurements and what components were not yet added led to a complex book-keeping exercise. The differences between the two SCT end-caps (reported in [2]) greatly exceeded the known differences and hence it was impossible to be confident in the measured weights. Furthermore the uncertainties in the measurements and arising from the subtraction of the TRT weight exceeded those from the bottom-up estimates.

Distributions of the numbers of radiation lengths for the inner detector can be found in [2]. Figure 58 shows the distribution for the SCT end-cap only. The material for a single disk (including modules and services) at normal incidence is $3.8 \%$ of a radiation length.

\subsection{Expected tracking performance}

The expected tracking performance for the inner detector is documented in [2]. When collision data have been collected, analysed and understood, more complete performance studies will be documented. 

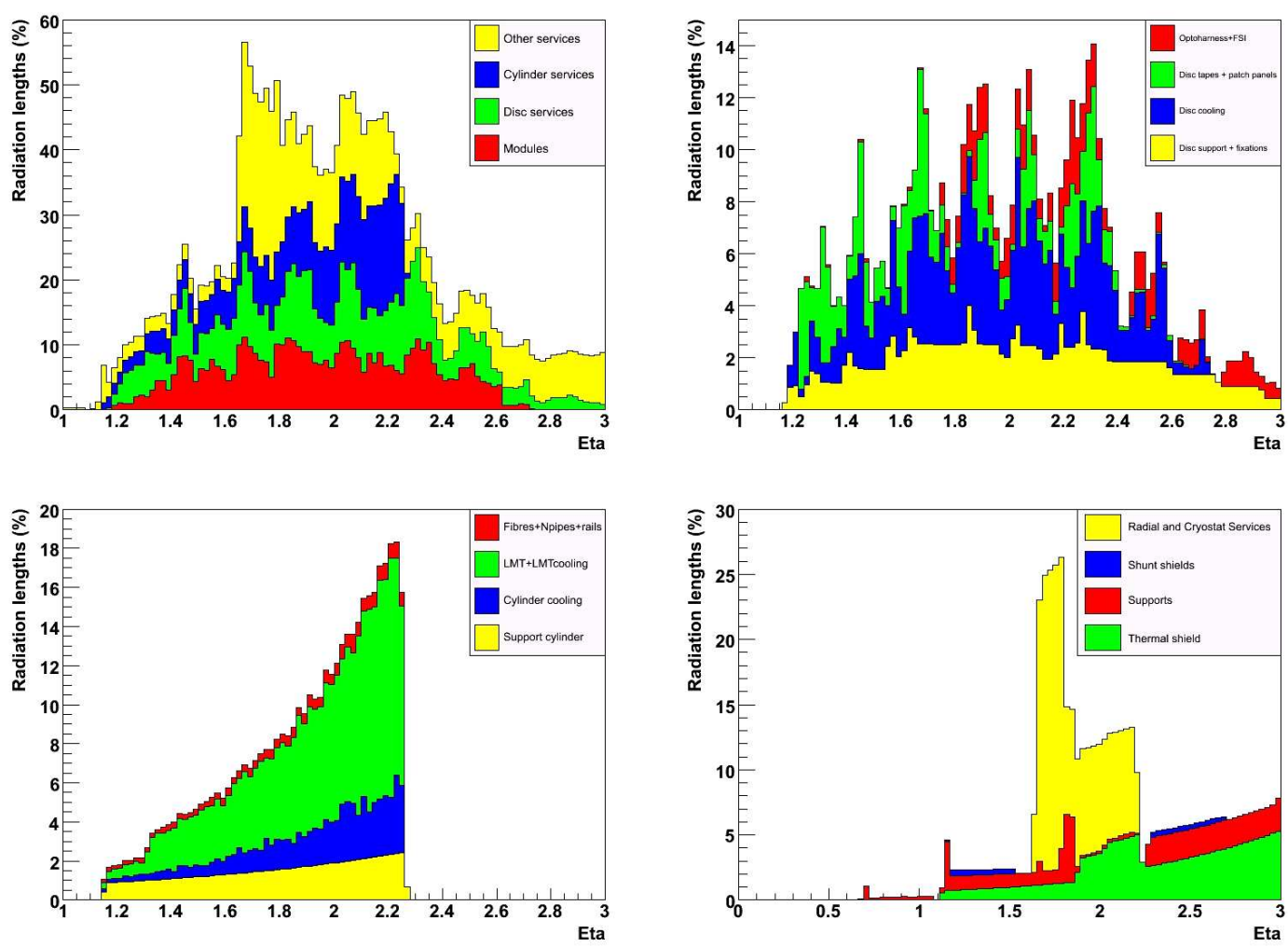

Figure 58. Number of radiation lengths crossed by a particle coming from the origin (no beam-spot smearing) and traversing the SCT end-cap. The plots show the components for the compete end-cap (top-left), disks (top-right), cylinder services (bottom-left) and external services (excluding PPF1) (bottom-right). Distributions are shown as a function of the pseudorapidity, defined in terms of the polar angle: $\eta=-\ln (\tan (\theta / 2))$.

\section{Conclusions}

The engineering for the ATLAS SCT end-caps will have been completed early in 2008 .

1. Two stable structures have been created to hold the 1976 silicon modules.

2. The mechanical tolerances have been achieved in almost all cases; the positions of the modules within the end-caps are known to $\mathrm{O}(100) \mu \mathrm{m}$ in the $\mathrm{x}-\mathrm{y}$ plane.

3. The end-caps will be capable of providing a suitable environment for the modules, in particular with respect to the thermal and electrical requirements.

4. The module performance, both in terms of number of operational channels and electrical characteristics, appears to be little affected by their incorporation into the end-caps.

5. Due to careful assembly, integration, checking and replacement where necessary, the endcaps are very close to fully functional. 
6. The material budget is quite large, but this is unavoidable in the light of the other constraints (mechanical stability, on-detector readout, thermal performance).

The engineering project has been a large endeavour involving many engineers, technicians and physicists. The engineering, in particular that associated with services, is not very glamorous compared to the design of silicon detectors, and yet without it, the detectors cannot work. Furthermore, the challenges involved in the engineering design and implementation have been as great as any other part of the experiment and collaborations forget this at their peril. It is all too easy to ignore the huge amounts of manpower, money and time in undertaking the engineering for a project of this scale and it is not made easier by all the external constraints which add greatly to the complexity, requiring components at the edge of what is technical realisable.

Despite the bright ideas of the ATLAS physicists, it is a credit to our engineers that this project has been successfully realised.

\section{Acknowledgments}

We are greatly indebted to all the technical staff who worked on the end-cap engineering project from the ATLAS SCT Institutes. We acknowledge the support of the funding authorities of the collaborating institutes including the Spanish National Programme for Particle Physics; the Research Council of Norway; the Science and Technology Facilities Council of the United Kingdom; the Polish Ministry of Education and Science; the German Ministry of Science; the Swiss National Science Foundation; the State Secretariat for Education and Research and the Canton of Geneva; the Slovenian Research Agency and the Ministry of Higher Education, Science and Technology of the Republic of Slovenia; the Ministry of Education, Culture, Sports, Science and Technology of Japan; the Japan Society for the Promotion of Science; the Office of High Energy Physics of the United States Department of Energy; the United States National Science Foundation; the Australian Research Council (ARC) and Department of Education, Science and Training (DEST); the Dutch Foundation for Fundamental Research on Matter (FOM); the Netherlands Organisation for Scientific Research (NWO); the Ministry of Education, Youth and Sports of the Czech Republic; the National Science Council of Taiwan; the Swedish Research Council.

\section{A. Glues used}

All the following are radiation hard.

Araldite 2011. Two-part epoxy adhesive, for general purpose use for small area bonds between various materials. Also used for large-area bonds where cold curing is required. Used for bonding parts to the support structure, bonding the aluminium skins and polyimide heaters to the thermal enclosure and sealing the friable surfaces of the Airex $\AA$ foam.

RS4 cyanate-ester adhesive film. ${ }^{56}$ An areal adhesive, suitable for bonding of stable composite structures due to its low CTE and CME. Sensitive to moisture during curing. Used for co-curing

\footnotetext{
${ }^{56} \mathrm{RS} 4$ cyanate-ester adhesive film, manufactured by YLA Inc..
} 
of the YSH-50A/RS3 facesheets to the Korex $\AA$ core of the support cylinder.

FM73U ${ }^{57}$ Unsupported epoxy film-adhesive. A less moisture-sensitive, composite, areal adhesive than the RS4. Has higher CTE and CME properties. Used for bonding pre-cured YSH-50A/RS3 facesheets to the KOREX core of the front and rear supports.

Techsil. ${ }^{58}$ Two-part silicone sealant. A soft, weak sealant with very little adhesive strength; sets to a rubbery state. Low viscosity, so best used for leak-sealing small gaps in inaccessible areas. Initially used for sealing in services, but was replaced by Tempflex.

Tempflex. ${ }^{59}$ Single-part silicone sealant. Strongly adhesive in comparison to Techsil and used to bond parts with a sealed but flexible connection. Used for sealing and bonding between outer thermal enclosure components.

\section{B. Lessons learnt}

In designing the SCT end-cap, various decisions needed to be made. In retrospect, and in particular in the light of problems constructing the SCT, it might have been better to consider different approaches. In this section, some of these areas are highlighted; nevertheless, it is not obvious that these alternatives would necessarily be better.

\section{B.1 Layout}

To improve the track parameter resolution and reduce correlations between the parameters, the stereo orientation alternates from disk to disk. This has led to a large number of "flavours" of disks and their services. This made the design and assembly more complicated, requiring more drawings and the unit cost of components was higher. This configuration actually led to a design error for the barrel power tapes.

The last disk, disk 9, was rotated to place the silicon at the largest pseudorapidity. This complicated the design of the patch panels.

In general, it would be worth trying to keep all the disks as similar as possible to minimise design effort, cost and minimise the possibility for mistakes.

The modularity of 52, 40 and 40 modules for the outer, middle and inner rings arose from the desire to extract silicon detectors from round 4-inch wafers which maximised the use of the surface area and reduced the cost. This resulted in very many "flavours" of services being required, each of fairly complicated design. Furthermore, in having an odd number of outer modules in a quadrant, some of the tooling needed to be doubled up. Some of the costs savings on silicon may have been lost in the complexity of the services and it is probable that the mass of services is greater than it might have been otherwise. It would be worth considering a design which had a much higher degree

\footnotetext{
${ }^{57}$ FM73U, manufactured by Cytec Engineered Materials.

${ }^{58}$ Techsil 6166 RTV silicone rubber, manufactured by Techsil Ltd..

${ }^{59}$ Tempflex 5145, manufactured by Loctite.
} 
of rotation symmetry for the disks - the current design only has 4-fold rotation symmetry. ${ }^{60}$ It would be attractive to consider a "pointing" geometry with equal numbers of modules in each ring.

\section{B.2 Disk services}

The original intention had been to manufacture aluminium cooling circuits with aluminium cooling blocks. This worked well using tubing of $200 \mu \mathrm{m}$ wall thickness and the cooling blocks were easy to manufacture using $\mathrm{CNC}$ machining. The problem was how to connect the blocks to the pipes with a low thermal resistance. Soldering on selectively $\mathrm{Cu}$-plated areas worked well, but produced an interface between two metals of very different electrochemical potential which was shown to be susceptible to corrosion in the presence of moisture - this was especially true at the edge of the sputtering region, where pin-holes formed. For this reason, this solution was dropped and replaced with $\mathrm{Cu}-\mathrm{Ni}$. Nevertheless, it would be worth investigating all-aluminium welding or soft-soldering techniques to avoid corrosion. Unfortunately, there is always a risk of corrosion arising further downstream at the junction between dissimilar metals.

Carbon-carbon was chosen for the cooling blocks. While this material potentially has good thermal conductivity in certain directions, the conductivity is variable and it proved difficult to envisage simple QA procedures to select good material. Furthermore, the material was quite difficult to machine because of its softness and intrinsic fault lines. The resultant blocks were quite delicate and prone to breakages during assembly to the pads on the disks.

Block positioning as described for EC-C in section 2.2.3 was a difficult and time consuming operation. The mounting screws had to be sufficiently tight to hold the block down onto the mounting pad and to overcome the natural flexibility of the pipe. The only item which could be held was the block rather than the thin walled pipe and there was only very restricted access under the optics. Since the precise placement of the blocks was critical process, it would have benefited from much more prototyping. The use of a machined plate for the disks of EC-A was quicker, but relied on very accurate machining of the plate; and care was needed to avoid pulling the blocks off the disk or gluing the plate to the disk.

The design of the cooling circuits required a number of soldered joints to be made after mounting on the disks; this prevented testing prior to assembly. Consequently, it was impossible to obtain complete access to these joints for either soldering or for visual examination. The layout of the pipes, with both sides of the disk sharing common connectors, made it difficult and time consuming to find any leaks. Any leaks which were found meant that the cycle of cleaning and testing had to be repeated. This process denied any access for other installation operations, which impacted on the timescales. These problems stress the importance of having completed, tested modular components which can be used in the assembly.

There was some consideration given to a design where cooling pipes would run circumferentially around the disks. To cope with contraction of the circuits, this required sliding joints and the concern was that either the grease joint would fail or the modules would be pulled out of position. Although this design had less material, it was abandoned in favour of the "wiggly" circuit design described in section 2.2.1.1, since the latter naturally incorporated stress relief.

\footnotetext{
${ }^{60}$ This is not quite true for the cooling circuits for the outer modules.
} 
To reduce the possibility of corrosion at PPF0 due to the choice of aluminium seals (see section 2.2.1) it would be desirable to use copper seals with sturdier housing in order to supply the required compression of the seals.

The FSI system proved to be very delicate and there was no way envisaged during assembly to check its integrity. The quartz rods in the jewels were chosen to reduce thermal distortions, however they were exceptionally fragile and prone to shattering. In retrospect, some other low-CTE material should have been chosen.

Putting delicate fibres on the disks where they get damaged and cannot be replaced because they are under cooling circuits was a mistake. The VCSEL's ${ }^{61}$ are very sensitive to electrostatic discharges (ESD), so consequently we now have a significant number of dead VCSEL's on the disks and we must rely on the in-built redundancy to ensure that there is no loss of signal. It might be better to carry electrical signals from the modules to the edge of the disks and then convert to fibre. Due to the fragility and inaccessibility of the services, a large portion of the disk build-time was spent testing and re-testing services (maybe $30 \%$ prior to module mounting). Unfortunately the continual connection and re-connection at all the interfaces has increased the likelihood of failure. More thought should have been given to the overall testing regime: either to improve the accessibility of services, enabling them to be swapped more easily late in the process, or by making them all more robust, reducing the need for continual testing. A more robust design would use electrical connections from the modules to a nearby optoelectronic interface. This would avoid having fragile fibres on the disks and would allow the optoelectronics to be located in regions, such that they could be replaced more easily in the event of failures during assembly.

\section{B.3 Assembly}

The flexible gas and grounding-and-shielding membranes were conceptually simple and low mass. However, in the event of access being required to the insides of the end-cap, the membranes will undoubtedly be destroyed, requiring spares to be made. While the soldering technique for connecting the outside of the Faraday shield has proved very adaptable and straightforward, it is time consuming to seal or unseal the components. The use of copper adds to the number of radiation lengths it would be worthwhile to investigate welding techniques for aluminium foil which could be used in situ without risk to the other components.

The concept for sealing the end-cap relied heavily on the application of Tempflex $\AA$ and Techsil $\AA$ sealants. These are messy and required in poorly defined amounts. Nevertheless, the approach was flexible and there is some inevitability that loose sealant will be required to block sources of leaks. The use of Tempflex $\AA$ was more suitable in some applications than in others. It was least successful when the zone of application was large and poorly defined, such as between the power tapes on the STFT. Trying to identify and seal leaks proved very difficult in the presence of many complicated services and many interfaces. The sealing strategy would benefit from more careful thought and prototyping.

There is a conflict between the requirements of building low-mass, thin-walled cooling circuits and the rigidity needed to provide firm seals. This would benefit from much greater investigation and every effort should be made to keep crucial connections outside relatively inaccessible regions:

\footnotetext{
${ }^{61}$ Electronic components on the module hybrid which convert electrical signals to optical signals.
} 
it is not good to have the connectors at the end of the cooling interconnects on the support cylinder buried inside the STFT. This meant that it was not possible to seal up the thermal enclosures in SR1 and make definitive tests of the leak-tightness before inserting the end-caps into ATLAS.

A lot of effort went into designing low-mass cooling connectors to fit in very restricted spaces. Instead there is sense in using off-the-shelf connectors wherever possible.

Semi-rigid cooling circuits both on the support cylinder and from the SCT end-cap to PPF1 were designed to tight tolerances. However, during the assembly, these tolerances could not be achieved. S-bends were added later when space become available due to the cancellation of the final TRT wheels (see section 1.4). These provided the flexibility to connect all the cooling circuits. It would have been better not to assume the tolerances would be met in such a complicated system and build in sufficient flexibility at the very start of the design process.

While great care was taken when weighing the end-caps, there was a much greater discrepancy between the two end-caps than was credible. When weighing large assemblies, it is important to understand all the temporary components which have been added and which final components are missing when the measurement is undertaken. It is important to cross-check with expectations at the same time as the measurements are made, in case measurements need to be repeated.

\section{References}

ATLAS engineering documents can be obtained from: https://edms.cern.ch/cedar/plsql/edmsatlas.home.

ATLAS Notes can be obtained from: http://cdsweb.cern.ch/collection/ATLAS.

[1] The ATLAS collaboration, Inner Detector Technical Design Report, Vol 2, CERN/LHCC/97-17 (1997).

[2] The ATLAS collaboration, The ATLAS Experiment at the CERN Large Hadron Collider, submitted to JINST.

[3] The ATLAS collaboration, Magnet System Technical Design Report, Vol 1, CERN/LHCC/97-18 (1997).

[4] A. Abdesselam et al., The ATLAS SemiConductor Tracker End-cap Module, Nucl. Instrum. Meth. A $575(2007) 353$.

[5] The ATLAS collaboration, Inner Detector Technical Design Report, Vol 1, CERN/LHCC/97-16 (1997).

[6] S. Haywood, Determination of SCT Wheel Positions, ATLAS Engineering Document ATL-IS-ES-0080 (2002).

[7] S. Haywood, Stereo Definitions for the SCT, ATLAS Engineering Document ATL-IS-ES-0077 (2002).

[8] S. Snow and A. Weidberg, Alignment requirements for the inner detector, ATLAS Note INDET-NOTE-160.

[9] The ATLAS collaboration, Detector and Physics Performance Technical Design Report, Vol 1, CERN/LHCC/99-14 (1999).

[10] N. Hessey, Physics Requirements for End-cap Module Placement Tolerances, ATLAS Engineering Document ATL-IS-ER-0027 (2001). 
[11] S. Gibson et al., ATLAS SCT End-cap Alignment System Layout, ATLAS Engineering Document ATL-IS-ES-0081 (2005).

[12] P. Coe et al., Frequency scanning interferometry in ATLAS, Meas. Sci. Technol. 15 (2004) 2175.

[13] S. Gibson et al., Coordinate measurement in 2-D and 3-D geometry using frequency scanning interferometry, Opt. Laser Eng. 43 (2005) 815.

[14] M. Tavlet et al., Compilation of Radiation Damage Test Data, Part II, 2nd edition: Thermoset and thermoplastic resins, composite materials, CERN TIS 98-01 (1998).

[15] I. Dawson et al., Radioactivation of silicon tracker modules in high-luminosity hadron collider radiation environments, Nucl. Instrum. Meth. A 515 (2003) 422.

[16] H. Ott, Noise Reduction Techniques in Electronic Systems, Wiley (1988).

[17] The Use of Plastic and other Non-Metallic Materials at CERN with respect to Fire Safety and Radiation Resistance, CERN TIS IS 95-41 (1995).

[18] S. Snow, Magnetic Material in the Inner Detector, ATLAS Engineering Document ATL-IS- ES-0118 (2003).

[19] V. Vacek et al., Perfluorocarbons and Their Use in Cooling Systems for Semiconductor Particle Detectors, Fluid Phase Equilibria 174 (2000) 191.

[20] M. Olcese et al., Evaporative Cooling System for the ATLAS Inner Detector, to be submitted to JINST.

[21] P. Werneke, Performance Requirements of Disks and Inserts, ATLAS Engineering Document ATL-ISES-0041 (2000).

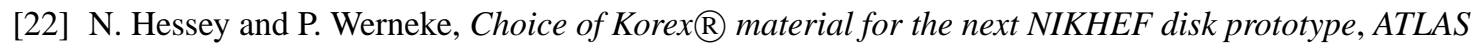
Engineering Document ATL-IS- ER-0029 (2001).

[23] N. Hessey et al., SCT End-cap Disc Preparation for Services, ATLAS Engineering Document ATL-ISAN-0003 (2001).

[24] S. Haywood, XY Metrology of SCT End-cap Disc 9C, ATLAS Engineering Document ATL-ISER-0051 (2003).

[25] Q. Snippe, Structural Analysis of the End-cap Discs, ATLAS Engineering Document ATL-ISEA-0004 (2003).

[26] I. Wilmut et al., SCT End-cap Disc Services Design, ATLAS Engineering Document ATL-IS-ER-0016 (2003).

[27] R. Fowler, CFD Simulations of Convective Heat Transfer in the ATLAS SCT End-cap, ATLAS Engineering Document ATL-IS-ER-00020 (2001).

[28] CFX 4.2 User Manuals, CFX International, Harwell, Didcot, UK (1997).

[29] I. Duerdoth et al., Measurements of Convection between the Disks of the SCT End-cap, ATLAS Engineering Document ATL-IS-TR-0010 (2004).

[30] V. O'Shea, SCT Forward Power Tape from PPFO to Module, ATLAS Engineering Document ATL-ISES-0082 (2003).

[31] A. Abdesselam et al., The optical links of the ATLAS SemiConductor Tracker, 2007 JINST 2 P09003.

[32] A. Abdesselam et al., The Detector Control System of the ATLAS SemiConductor Tracker during Macro-Assembly and Integration, to be submitted to JINST. 
[33] R. Brenner et al., Evaluation of a Humidity Sensor for Use in an Environment Exposed to Radiation, J. Test. Eval. 35 (2007) No. 5 .

[34] J. Foster et al., SCT Endcap Geometry and Surveys, ATLAS Note ATL-INDET-PUB-2007-004.

[35] ATLAS SCT collaboration, P.W. Philips et al., Functional Testing of the ATLAS SCT Barrels, Nucl. Instrum. Meth. A 570 (2007) 230.

[36] J. Bohm et. al., Power supply and power distribution system for the ATLAS silicon strip detectors, CERN-2001-005 (2001).

[37] M. Chu et al., The off-detector opto-electronics for the optical links of the ATLAS Semiconductor Tracker and Pixel detector, Nucl. Instrum. Meth. A 530 (2004) 293.

[38] J. Butterworth et al., TIM (TTC interface module) for ATLAS SCT and pixel readout electronics, CERN-2001-005 (2001).

[39] J. Tarrant et al., SCT End-cap Support Structure, ATLAS Engineering Document ATL-IS- ER-0017 (2001).

[40] C. Nelson, FE Analysis of SCT End-cap Support Structure, ATLAS Engineering Document ATL-ISEA-0001 (2001).

[41] C. Nelson, SCT End-cap Support Structure - Frequency Response Analysis, ATLAS Engineering Document ATL-IS- EA-0002 (2001).

[42] V. Juraylev et al., Investigations of Power and Spatial Correlation Characteristics of Seismic Vibrations in the CERN LEP Tunnel for Linear Collider Studies, 1993 CERN-SL-93-53.

[43] A. Weidberg, The Production of the SCT Optical Links, Proceedings of the eleventh Workshop on Electronics for LHC and Future Experiments, Heidelberg, Germany, 12-16 September 2005, CERN-2005-011 (2005).

[44] S. Haywood, Transportation for the ATLAS SCT End-cap (EC-C), ATLAS Engineering Document ATL-IS- CS-0016 (2005).

[45] J. Tarrant et al., SCT End-cap Thermal Enclosures, ATLAS Engineering Document ATL-IS-ER-0018 (2004).

[46] F. Hartjes, Moisture Management in the ATLAS SCT and Pixel Tracker Using Dry Nitrogen Flow, ATLAS Engineering Document ATL-IS-EN-0015 (2003).

[47] P. Ford, SCT End-cap Thermal Enclosure Post-FDR Prototyping and Analysis, ATLAS Engineering Document ATL-IS-ER-0058 (2005).

[48] P. Ford, Heat Transfer Analysis of the Thermal Enclosure, ATLAS Engineering Document ATL-IS-EA-0006 (2004).

[49] P. Werneke and J. Tarrant, Transport of the SCT End-cap from SRI to the ATLAS Pit, ATLAS Engineering Document ATL-IS-ES-0117 (2007).

[50] B. Smith, SCT End-cap Integration Procedure, ATLAS Engineering Document ATL-IS-ER-0067 (2005).

[51] S. Haywood et al., ATLAS SCT End-cap Grounding and Shielding, ATLAS Engineering Document ATL-IS-EN-0014 (2007). 